\title{
A Mathematical One-Dimensional Model of Supercooling Solidification
}

\author{
By
}

\author{
Tatsuo NoGI*
}

\section{§. Introduction}

As well known in physics, an equilibrium condition on a contact surface separating two parts of a pure metal/material, solid and liquid part, is usally given by the equation

$$
(\Delta F)_{T_{E}} \equiv\left(F_{L}\right)_{T_{E}}-\left(F_{S}\right)_{T_{E}}=0
$$

where the suffix $T_{E}$ indicates quantities at the equilibrium temperature $T_{E}$, and $F_{L}$ and $F_{S}$ are the free energy of liquid $(L)$ and $\operatorname{solid}(S)$ respectively:

$$
F_{L} \equiv E_{L}-T S_{L}, \quad F_{S} \equiv E_{S}-T S_{S}
$$

They may be considered at any degree $T^{\circ} \mathrm{K}$ of temperature, and $E_{L}$ and $E_{S}$ are the internal energy, and further $S_{L}$ and $S_{S}$ are the entropy.

On the other hand, at any temperature different from $T_{E}, \Delta F$ is not zero, and it is given by the formula

$$
\Delta F=\Delta E-T \Delta S=\Delta E \frac{T_{E}-T}{T_{E}}=L \frac{T_{E}-T}{T_{E}},
$$

where it is assumed that the difference $E$ and $S$ do not depend on $T$, and $L=\Delta E$ is called latent heat. In general, solidification may occur only for the case of $T<T_{E}$, since $F_{L}>F_{S}$. Hence, it seems natural to consider that only supercooling state allows solidification. But, in usally setting of the Stefan problem, it is assumed that solidification occur just at the equilibrium temperature.

In this paper, such supercooling solidification is considered, and its mathematical one-dimensional model is proposed. An important

Communicated by S. Hitotumatu, October 26, 1984.

* Department of Applied Mathematics and Physics, Faculty of Engineering, Kyoto University. 
assumption is that a rate of solidification on a contact surface is linearly proportional to $\Delta F=F_{L}-F_{S}$, i. e., the contact surface speed is a constant times the supercooling degree $T_{E}-T$ on each corresponding point of the surface.

Only the following case is considered; some supercooling liquid is first held quietly in a straight tube with a length $l$, and then a solidification process starts: it proceeds from one side bottom of the tube to the other side. For simplicity, we assume that the temperature distribution on each cross section perpendicular to the axis of the tube is uniform, and that solidification continues in the onedimensional way. The speed of the surface is then given by the formula

$$
\dot{y}(t)=\frac{d y(t)}{d t}=K\left(T_{E}-T\right),
$$

where $y(t)$ is the distance between the start point and the contact surface at the time $t, K$ is a constant and $T$ is the temperature on the surface. The solidification process produces a quantity of latent heat, $L \rho \dot{y}(t)$ per a unit time and per a unit cross section, where $L$ is the latent heat per a unit mass and $\rho$ is the density of the concerning material which, we assume, is a common constant for liquid and solid. Produced heat by solidification is diffused into both liquid and solid. The heat balance equation is then given as follows:

$$
L \rho \dot{y}(t)=k_{S} \frac{\partial T}{\partial x}(y(t)-0, t)-k_{L} \frac{\partial T}{\partial x}(y(t)+0, t),
$$

where $k_{S}$ and $k_{L}$ are heat conductivity coefficients of solid and liquid respectively.

Diffusion process in the solid and liquid state, we assume as usual, is expressed by the heat equation

$$
\rho c_{S} \frac{\partial T}{\partial t}=k_{S} \frac{\partial^{2} T}{\partial x^{2}} \quad \text { (in the solid) }
$$

and

$$
\rho c_{L} \frac{\partial T}{\partial t}=k_{L} \frac{\partial^{2} T}{\partial x^{2}} \quad \text { (in the liquid), }
$$

where $c_{S}$ and $c_{L}$ are specific heat.

Typical initial and boundary condition are the followings;

$$
T(x, 0)=T_{A},
$$




$$
T(0, t)=T_{1}(t), \quad T(l, t)=T_{2}(t),
$$

where $T_{A}, T_{1}(t)$ and $T_{2}(t)$ are a given constant and given functions.

The variable change

$$
T-T_{A} \rightarrow u, \quad \frac{x}{l} \rightarrow x, \quad \frac{y}{l} \rightarrow y
$$

reduces the above equations and conditions to the following normalized form:

$$
\begin{aligned}
c_{1} \frac{\partial u}{\partial t} & =a_{1} \frac{\partial^{2} u}{\partial x^{2}} \quad(0<x<y(t), t>0), \\
c_{2} \frac{\partial u}{\partial t} & =a_{2} \frac{\partial^{2} u}{\partial x^{2}} \quad(y(t)<x<1, t>0) \\
b \dot{y}(t) & =a_{1} \frac{\partial u}{\partial x}(y(t)-0, t)-a_{2} \frac{\partial u}{\partial x}(y(t)+0, t) \\
& =\alpha\left(u_{E}-u(y(t), t)\right) \quad(t>0), \\
y(0) & =0, \\
u(x, 0) & =\phi(x) \quad(0<x<1), \\
u(0, t) & =f_{1}(t), \quad u(1, t)=f_{2}(t) \quad(t>0),
\end{aligned}
$$

where $c_{1}=\rho c_{S} l, c_{2}=\rho c_{L} l, a_{1}=k_{S} / l, a_{2}=k_{L} / l, b=L \rho l, \alpha=L \rho K, u_{E}=T_{E}-T_{A}$, $f_{1}(t)=T_{1}(t)-T_{A}, f_{2}(t)=T_{2}(t)-T_{A}$ and $\phi(x)=0$. It must be here noticed that by the physical reason

$$
a_{1}>a_{2}, \quad c_{1}<c_{2} \text {. }
$$

In this paper it will be proved that the problem (1.7)-(1.13) has a unique solution under some conditions on data, while general initial data $\phi(x) \geqq 0$ being considered. In $\S 2$, a difference scheme is introduced. It gives a sequence of approximate solutions of the above problem. Some energy estimates of those solutions are also given. In $\S 3$, it is shown that a local solution of the problem is obtained as a limit of the sequence of approximate solutions. In $\S 4$, it is seen by continuing local solutions successively that a global solution exists certainly as far as liquid state remains. In $\S 5$, its uniqueness is proved under the condition that initial supercooling is not so much. Appendix A is to give estimations of the so-called Bernstein type for a solution of heat difference scheme. Appendix B is to comment an Imbedding Theorem. For some numerical examples, see [1].

Our problem is formally similar to the so-called Muskat's problem 
which relates to physical processes of filtration in porous media. It has the internal boundary condition

$$
\left(\frac{1}{a_{1}}-\frac{1}{a_{2}}\right) u(y(t), t) \dot{y}(t)+\frac{1}{c_{1}} \frac{\partial u}{\partial x}(y(t)-0, t)=\frac{1}{c_{2}} \frac{\partial u}{\partial x}(y(t)+0, t)
$$

and

$$
\dot{y}(t)=\alpha u(y(t), t),
$$

instead of (1.9) in our case. Such problems have been solved by W. Fulks and R. B. Guenther [2], I. Pawlow [3], etc. Their proofs of existence and uniquness theorem rely on the reformulation using integral equations. We believe that our method will solve such problems as well, under weaker conditions upon data.

\section{§2. Difference Scheme and Its Solution}

2.1. We will give a difference scheme which gives a sequence of approximate solutions. It is considered on a net of rectangular meshes which is the same as used in [4] for solving a two phase Stefan problem. In fact, it has a uniform space width $h$ and variable time steps $\left\{k_{n}\right\} \quad(n=1,2,3, \ldots)$. The time steps are assumed to be unknown a priori and to be determined in a process of solving by the rule that $h / k_{n}$ may give the gradient of the contact boundary $x=y(t)$ at every time $t=t_{n}$, so that the contact boundary may cross every line of the ordinate $x=x_{j}$ only at every corresponding mesh point. Then, it is convenient to introduce discrete coordinates like

$$
\begin{aligned}
& x_{j}=j h \quad(j=0,1,2, \ldots, M ; M h=1), \\
& t_{n}=\sum_{p=1}^{n} k_{p} \quad(n=1,2,3, \ldots)
\end{aligned}
$$

and net functions like $y_{n}$ and $u_{j}^{n}$ which correspond clearly to $y\left(t_{n}\right)$ and $u\left(x_{j}, t_{n}\right)$ respectively. By the rule mentioned above, it is admitted to put

$$
y_{n}=J_{n} h \quad(n=0,1,2,3, \ldots),
$$

where $\left\{J_{n}\right\}$ is a sequence of integers such that $J_{n+1}=J_{n}+1 \quad(n=$ $0,1,2,3, \ldots)$. Though it is natural to take $J_{0}=0$ since $y(0)=0$, we take

$$
J_{0}=1 \quad\left(y_{0}=h\right),
$$

allowing the errors $O(h)$, in order to avoid another procedure at the 
initial stage of our algorithm and simplify later argument. Express the inverse function of $x=J_{n} h$ by

$$
t=t_{N_{j}} \quad(j=1,2,3, \ldots) \text {. }
$$

Let's introduce divided differences as usual:

$$
\begin{aligned}
& \left(u_{j}^{n}\right)_{x}=\left(u_{j+1}^{n}-u_{j}^{n}\right) / h, \quad\left(u_{j}^{n}\right)_{\bar{x}}=\left(u_{j}^{n}-u_{j-1}^{n}\right) / h, \\
& \left(u_{j}^{n}\right)_{x \bar{x}}=\left(u_{j+1}^{n}-2 u_{j}^{n}+u_{j-1}^{n}\right) / h^{2}, \quad\left(u_{j}^{n}\right)_{\bar{t}}=\left(u_{j}^{n}-u_{j}^{n-1}\right) / k_{n}, \text { etc。 }
\end{aligned}
$$

2. 2. The difference scheme used to solve our problem is as follows:

$$
\begin{aligned}
& J_{0}=1, \quad u_{j}^{0}=\phi_{j} \quad(j=1,2, \ldots, M-1), \\
& b \frac{h}{k_{n}}=\alpha\left(u_{E}-u_{J_{n-1}}^{n-1}\right) \\
& c_{1}\left(u_{j}^{n}\right)_{\bar{t}}=a_{1}\left(u_{j}^{n}\right)_{x \bar{x}} \quad\left(j=1,2, \ldots, J_{n}-1\right), \\
& a_{1}\left(u_{J_{n}}^{n}\right)_{\bar{x}}-a_{2}\left(u_{J_{n}}^{n}\right)_{x}=\alpha\left(u_{E}-u_{J_{n}}^{n}\right), \\
& c_{2}\left(u_{j}^{n}\right)_{\bar{t}}=a_{2}\left(u_{j}^{n}\right)_{x \bar{x}} \quad\left(j=J_{n}+1, J_{n}+2, \ldots, M-1\right), \\
& u_{0}^{n}=f_{1}^{n}, \quad u_{M}^{n}=f_{2}^{n} \quad(n=1,2,3, \ldots) .
\end{aligned}
$$

In this scheme, $\left\{k_{n}\right\}$ and $\left\{u_{j}^{n}\right\}$ are unknown variables to be found, while $y(t)$ and $u(x, t)$ are unknown in the original problem of differential system.

The procedure to solve the above difference scheme starts from determining the first time step $k_{1}$ by $(2.6)$ and (2.7) with $n=1$. It gose next to find $u_{j}^{1}$ by solving the linear algebraic system of $(2.8)-(2.11)$ with $n=1$. Certainly, the last system is solvable. The next step is to find $k_{2}$ and $u_{j}^{2}$, and the third to find $k_{3}$ and $u_{j}^{3}$ and so on. It is, of course, necessary for having positive time steps $k_{n}$ 's and continuing the above solution process successfully to assure the condition

$$
u_{J_{n}}^{n}<u_{E} \quad(n=0,1,2,3, \ldots) .
$$

2. 3. Lemma 2.1. Assume that

$$
0 \leqq \phi(x), f_{1}(t) \text { and } f_{2}(t)<u_{E} \text {. }
$$

Then, (2.12) and the followings hold:

$$
\begin{aligned}
& 0 \leqq u_{j}^{n}<u_{E} \quad(j=1,2, \ldots, M-1 ; n=1,2,3, \ldots) \\
& h<\frac{\alpha u_{E}}{b} k_{n} \quad(n=1,2,3, \ldots),
\end{aligned}
$$




$$
\left(N_{1}-N\right) h<\frac{\alpha u_{E}}{b}\left(t_{N_{1}}-t_{N}\right) \quad\left(N_{1}>N ; N_{1}, N=0,1,2, \ldots\right) .
$$

Proof. The statement follows from the well-known maximum principle of the implicit difference scheme for the heat equation immediately. In fact, suppose that $u_{J_{n}}^{n}<u_{E}$ for $n=1,2,3, \ldots, N$ and $u_{J_{N}}^{N}$ first happens to take a value $\geqq u_{E}$. Just by the principle, we then have $u_{j}^{n}<u_{E}$ for all $j=1,2, \ldots, M-1$ and $n=1,2, \ldots, N-1$. Further, by the assumption and (2.9), we have $a_{1}\left(u_{J_{N}}^{N}\right)_{\bar{x}}-a_{2}\left(u_{J_{N}}^{N}\right)_{x} \leqq 0$. The principle, on the other hand, yields $\left(u_{J_{N}}^{N}\right)_{\bar{x}}>0$ and $\left(u_{J_{N}}^{N}\right)_{x}<0$. This is a contradiction. So, we must have (2.12), and hence also (2.14) again by the principle. From (2.7) and (2.12), we get

$$
h=\frac{\alpha}{b}\left(u_{E}-u_{J_{n-1}}^{n-1}\right) k_{n}<\frac{\alpha u_{E}}{b} k_{n}
$$

and hence

$$
\left(N_{1}-N\right) h<\frac{\alpha u_{E}}{b} \sum_{n=N+1}^{N_{1}} k_{n}=\frac{\alpha u_{E}}{b}\left(t_{N_{1}}-t_{N}\right) .
$$

2.4. Lemma 2.2. Supbose that

(2.17) $\left|f_{1}(t)-f_{1}\left(t^{\prime}\right)\right|<H\left|t-t^{\prime}\right| \quad\left(H:\right.$ a constant, $\left.t, t^{\prime}>0\right)$, and $f_{1}(0)=0$, and that

(2.18) $\quad u_{J_{n}}^{n}<(1-\mu) u_{E} \quad(0<\mu<1)$ for $0 \leqq t_{n} \leqq t_{N-1}$.

Then, the inequality

$$
\left|u_{0 x}^{n}\right|<H_{1}=\frac{H}{\delta} \quad\left(\delta=\frac{\alpha \mu(1-\mu)}{b} u_{E}\right)
$$

holds for sufficiently small $h$ and $t_{N}$.

Proof. From (2.6) and (2.7), we have

$$
y_{n}=h+n h=h+\frac{\alpha}{b} \sum_{p=1}^{n} k_{p}\left(u_{E}-u_{J_{p-1}}^{p-1}\right) \text {. }
$$

Applying (2.12) and the assumption (2.18), we hence have

$$
h+\frac{\alpha}{b} \mu u_{E} t_{n}<y_{n}<h+\frac{\alpha}{b} u_{E} t_{n}
$$

for $0 \leqq t_{n} \leqq t_{N-1}$. Now, we fix a number $n_{0}(\leqq N)$ arbitrarily and consider an auxiliary function 


$$
\zeta_{n_{0}}\left(x_{j}, t_{n}\right)=f_{1}^{n_{0}}-H\left(t_{n_{0}}-t_{n}\right)-H_{1} x_{j}+\frac{c_{1}}{2 a_{1}} H x_{j}^{2} .
$$

As easily seen, it satisfies the equation $c_{1} \zeta_{\bar{t}}=a_{1} \zeta_{x \bar{x}}$ and the inequality

$$
\zeta_{n_{0}}\left(0, t_{n}\right)=f_{1}^{n_{0}}-H\left(t_{n_{0}}-t_{n}\right)<f_{1}^{n} \quad(\text { by } \quad(2.17))
$$

and

$$
\begin{aligned}
\zeta_{n_{0}}\left(y_{n}, t_{n}\right) & =f_{1}^{n_{0}}-H\left(t_{n_{0}}-t_{n}\right)-H_{1} y_{n}+\frac{\iota_{1}}{2 a_{1}} H y_{n}^{2} \\
& <f_{1}^{n}-H_{1} y_{n}\left(1-\frac{c_{1} H}{2 H_{1} a_{1}} y_{n}\right)
\end{aligned}
$$

for $n \leqq n_{0}$. Assume that

$$
h<h_{0}=\frac{a_{1} b}{c_{1} u_{E} \alpha} \quad \text { and } t_{N}<T_{0}=\frac{a_{1} b^{2}}{c_{1} \alpha^{2} u_{E}^{2}} .
$$

We then have

$$
1-\frac{c_{1} H}{2 H_{1} a_{1}} y_{n}>1-\mu
$$

So, we get

$$
\begin{aligned}
\zeta_{n_{0}}\left(y_{n}, t_{n}\right) & <f_{1}^{n}-(1-\mu) H_{1}\left(h+\frac{\alpha \mu}{b} u_{E} t_{n}\right) \\
& <f_{1}^{n}-\frac{\alpha \mu(1-\mu)}{b \delta} u_{E} H t_{n}<f_{1}^{n}-H t_{n}<0
\end{aligned}
$$

(see (2.23), (2.20), (2.19) and (2.17).) The condition (2.22) and (2.25) assure from the maximum principle that

$$
\zeta_{n_{0}}\left(x_{j}, t_{n}\right)<u_{j}^{n}
$$

in $\left\{0<x_{j}<y_{n}, 0<t_{n} \leqq t_{n_{0}}\right\}$. Putting $n=n_{0}$ and $x=x_{1}$ especially, we have

$$
\zeta_{n_{0}}\left(x_{1}, t_{n_{0}}\right)<u_{1}^{n_{0}}
$$

Since $\zeta_{n_{0}}\left(0, t_{n_{0}}\right)=u_{0}^{n_{0}}=f_{1}^{n_{0}}$, we hence get

$$
\left(\zeta_{n_{0}}\left(0, t_{n_{0}}\right)\right)_{x}<\left(u_{0}^{n_{0}^{0}}\right)_{x},
$$

that is,

$$
\left(u_{0}^{n_{0}}\right)_{x}>-H_{1}+\frac{c_{1}}{2 a_{1}} H h>-H_{1} .
$$


Similarly, by using another auxiliary function

$$
\zeta_{n_{0}}\left(x_{j}, t_{n}\right)=f_{1}^{n_{0}}+H\left(t_{n_{0}}-t_{n}\right)+H_{1} x_{j}-\frac{c_{1}}{2 a_{1}} H x_{j}^{2}
$$

we also have

$$
\left(u_{0}^{n_{0}}\right)_{x}<H_{1} \text {. }
$$

Since $n_{0}$ is selected arbitrarily, both (2.26) and (2.27) produce the desired inequality (2.19).

2.5. Now we will state a fundamental lemma for construction of a local solution.

Lemma 2. 3. Suppose that the data $f_{1}, f_{2}$ and $\phi$ satisfy (2.13), they are Lipshitz continuous, and $f_{1}(0)=f_{2}(0)=\phi(0)=0$. Then, for any given constant $\mu, 0<\mu<1$, there are positive constant $T_{1}$ and $K$ such that a

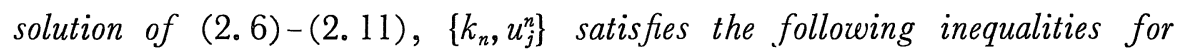
$0<t_{n}<t_{N}<T_{1}$ and $0<h<h_{0}, h_{0}$ being given in (2.24):

$$
\begin{gathered}
\sum_{n=1}^{N} k_{n} \sum_{j=1}^{M-1} h\left(u_{j \bar{t}}^{n}\right)^{2}+\sum_{j=0}^{M-1} h\left(u_{j x}^{N}\right)^{2}<K, \\
u_{J_{n}}^{n}<(1-\mu) u_{E}
\end{gathered}
$$

and

$$
\max _{p \leqq n} k_{p}<\frac{1}{\mu} \min _{p \leqq n} k_{p}
$$

The remained part of this section is devoted to the proof of Lemma 2.3. To get the energy inequality (2.28), we introduce a function $\gamma$ such that

$$
\gamma_{0}^{n}=f_{1 \bar{t}}^{n}, \quad \gamma_{M}^{n}=f_{2 \bar{t}}^{n} .
$$

By multiplying $(2.8)$ by $u_{j \bar{t}}^{n}-\gamma_{j}^{n}$, summing these products over $1 \leqq j \leqq J_{n}-1$ and $1 \leqq n \leqq N$ and taking summation by parts, we obtain

$$
\begin{aligned}
& c_{1} \sum_{n=1}^{N} k_{n} \sum_{j=1}^{J_{n}-1} h\left(u_{j \bar{t}}^{n}\right)^{2}-c_{1} \sum_{n=1}^{N} k_{n} \sum_{j=1}^{J_{n}-1} h u_{j \bar{t}}^{n} \gamma_{j}^{n}+a_{1} \sum_{n=1}^{N} k_{n} \sum_{j=1}^{J_{n}-1} h u_{j x}^{n} u_{j x \bar{t}}^{n} \\
& -a_{1} \sum_{n=1}^{N} k_{n} \sum_{j=1}^{J_{n}-1} h u_{j x}^{n} \gamma_{j x}^{n}-a_{1} \sum_{n=1}^{N} k_{n} u_{J_{n}-1 x}^{n}\left(u_{J_{n} \bar{t}}^{n}-\gamma_{J_{n}}^{n}\right)=0
\end{aligned}
$$

by (2.31). The third term is expanded like that 


$$
\begin{aligned}
a_{1} \sum_{n=1}^{N} k_{n} \sum_{j=1}^{J_{n}-1} h u_{j x}^{n} u_{j x \bar{t}}^{n}= & \frac{a_{1}}{2} \sum_{j=0}^{J_{N}-1} h \sum_{n=N_{j}+1}^{N}\left\{\left(u_{j x}^{n}\right)^{2}-\left(u_{j x}^{n-1}\right)^{2}+\left(u_{j x}^{n}-u_{j x}^{n-1}\right)^{2}\right\} \\
= & \frac{a_{1}}{2}\left\{\sum_{j=0}^{J_{N}-1} h\left(u_{j x}^{N}\right)^{2}-\sum_{j=0}^{J_{0}} h\left(u_{j x}^{0}\right)^{2}-\sum_{n=1}^{N-1} h\left(u_{J_{n} x}^{n}\right)^{2}\right. \\
& \left.+\sum_{n=1}^{N} k_{n}^{2} \sum_{j=0}^{J_{n}-1} h\left(u_{j x \bar{t}}^{n}\right)^{2}\right\} .
\end{aligned}
$$

The equation (2.32) becomes, hence and by (2.6),

$$
\begin{aligned}
& c_{1} \sum_{n=1}^{N} k_{n} \sum_{j=1}^{J_{n}-1} h\left(u_{j \bar{t}}^{n}\right)^{2}+\frac{a_{1}}{2} \sum_{j=0}^{J_{N}-1} h\left(u_{j x}^{N}\right)^{2}+\frac{a_{1}}{2} \sum_{n=1}^{N} k_{n}^{2} \sum_{j=0}^{J_{n}-1} h\left(u_{j x \bar{t}}^{n}\right)^{2} \\
& \quad-a_{1} \sum_{n=1}^{N} k_{n} u_{J_{n} \bar{x}}^{n}\left(u_{J_{n}}^{n}-\gamma_{J_{n}}^{n}\right) \\
& =\frac{a_{1}}{2} \sum_{n=1}^{N-1} h\left(u_{J_{n} x}^{n}\right)^{2}+c_{1} \sum_{n=1}^{N} k_{n} \sum_{j=1}^{J_{n}-1} h u_{j \bar{t}}^{n} \gamma_{j}^{n}+a_{1} \sum_{n=1}^{N} k_{n} \sum_{j=1}^{J_{n}-1} h u_{j x}^{n} \gamma_{j x}^{n} \\
& \quad+\frac{a_{1}}{2} \sum_{j=0}^{J_{0}} h \phi_{j x}^{2} .
\end{aligned}
$$

Similarly, the following equation follows from (2.10):

$$
\begin{aligned}
& c_{2} \sum_{n=1}^{N} k_{n} \sum_{j=J_{n}+1}^{M-1} h\left(u_{j \bar{t}}^{n}\right)^{2}+\frac{a_{2}}{2} \sum_{j=J_{N}}^{M-1} h\left(u_{j x}^{N}\right)^{2}+\frac{a_{2}}{2} \sum_{n=1}^{N} k_{n}^{2} \sum_{j=J_{n}}^{M-1} h\left(u_{j x \bar{t}}^{n}\right)^{2} \\
& \quad+a_{2} \sum_{n=1}^{N} k_{n} u_{J_{n} x}^{n}\left(u_{J_{n}}^{n}-\gamma_{J_{n}}^{n}\right) \\
& =-\frac{a_{2}}{2} \sum_{n=1}^{N-1} h\left(u_{J_{n}}^{n}\right)^{2}+c_{2} \sum_{n=1}^{N} k_{n} \sum_{j=J_{n}+1}^{M-1} h u_{j \bar{t}}^{n} \gamma_{j}^{n}+a_{2} \sum_{n=1}^{N} k_{n} \sum_{j=J_{n}}^{M-1} h u_{j x}^{n} \gamma_{j x}^{n} \\
& \quad+\frac{a_{2}}{2} \sum_{j=J_{0}+1}^{M-1} h \phi_{j x}^{2} .
\end{aligned}
$$

Adding (2.33) and (2.34) on their both sides produces the equation

$$
\begin{aligned}
c_{1} & \sum_{n=1}^{N} k_{n} \sum_{j=1}^{J_{n}-1} h\left(u_{j \bar{t}}^{n}\right)^{2}+c_{2} \sum_{n=1}^{N} k_{n} \sum_{j=J_{n}+1}^{M-1} h\left(u_{j \bar{t}}^{n}\right)^{2}+\frac{a_{1}}{2} \sum_{j=0}^{J_{N^{-1}}} h\left(u_{j x}^{N}\right)^{2} \\
& +\frac{a_{2}}{2} \sum_{j=J_{N}}^{M-1} h\left(u_{j x}^{N}\right)^{2}+\frac{a_{1}}{2} \sum_{n=1}^{N} k_{n}^{2} \sum_{j=0}^{J_{n}-1} h\left(u_{j x \bar{t}}^{n}\right)^{2} \\
& +\frac{a_{2}}{2} \sum_{n=1}^{N} k_{n}^{2} \sum_{j=J_{n}}^{M-1} h\left(u_{j x \bar{t}}^{n}\right)^{2}-\sum_{n=1}^{N} k_{n}\left(a_{1} u_{J_{n} \bar{x}}^{n}-a_{2} u_{J_{n^{x}}}^{n}\right)\left(u_{J_{n}}^{n}-\gamma_{J_{n}}^{n}\right)=
\end{aligned}
$$




$$
\begin{aligned}
& =\frac{1}{2}\left(a_{1}-a_{2}\right) \sum_{n=1}^{N-1} h\left(u_{J_{n} x}^{n}\right)^{2}+c_{1} \sum_{n=1}^{N} k_{n} \sum_{j=1}^{J_{n}-1} h u_{j i}^{n} \gamma_{j}^{n}+c_{2} \sum_{n=1}^{N} k_{n} \sum_{j=J_{n}+1}^{M-1} h u_{j i}^{n} \gamma_{j}^{n} \\
& +a_{1} \sum_{n=1}^{N} k_{n} \sum_{j=1}^{J_{n}{ }^{-1}} h u_{j x}^{n} \gamma_{j x}^{n}+a_{2} \sum_{n=1}^{N} k_{n} \sum_{j=J_{n}}^{M-1} h u_{j x}^{n} \gamma_{j x}^{n}+\frac{a_{1}}{2} \sum_{j=0}^{J_{0}} h \phi_{j x}^{2} \\
& +\frac{a_{2}}{2} \sum_{j=J_{0}+1}^{M-1} h \phi_{j x}^{2} .
\end{aligned}
$$

By using the condition (2.9), the last sum on the left hand side of (2.35) is expanded as follows;

$$
\begin{aligned}
-\sum_{n=1}^{N} & k_{n}\left(a_{1} u_{J_{n} \bar{x}}^{n}-a_{2} u_{J_{n}}^{n}\right)\left(u_{J_{n} \bar{t}}^{n}-\gamma_{J_{n}}^{n}\right) \\
= & -\alpha \sum_{n=1}^{N} k_{n}\left(u_{E}-u_{J_{n}}^{n}\right)\left(u_{J_{n}}^{n}-\gamma_{J_{n}}^{n}\right) \\
= & -\alpha \sum_{n=1}^{N}\left(u_{E}-u_{J_{n}}^{n}\right)\left(u_{J_{n}}^{n}-u_{J_{n}}^{n-1}\right)+\alpha \sum_{n=1}^{N} k_{n}\left(u_{E}-u_{J_{n}}^{n}\right) \gamma_{J_{n}}^{n} \\
= & -\alpha \sum_{n=1}^{N}\left(u_{E}-u_{J_{n}}^{n}\right)\left(u_{J_{n}}^{n}-u_{J_{n-1}}^{n-1}\right)+\alpha \sum_{n=1}^{N} h\left(u_{E}-u_{J_{n}}^{n}\right)\left(u_{J_{n-1}}^{n-1}\right)_{x} \\
& \quad+\alpha \sum_{n=1}^{N} k_{n}\left(u_{E}-u_{J_{n}}^{n}\right) \gamma_{J_{n}}^{n} .
\end{aligned}
$$

Notice here that

$$
u_{J_{n}}^{n}\left(u_{J_{n}}^{n}-u_{J_{n-1}}^{n-1}\right)=\frac{1}{2}\left[\left(u_{J_{n}}^{n}\right)^{2}-\left(u_{J_{n-1}}^{n-1}\right)^{2}+\left(u_{J_{n}}^{n}-u_{J_{n-1}}^{n-1}\right)^{2}\right] .
$$

Therefore, the first term of the last expression is equal to

$$
-\alpha u_{E}\left(u_{J_{N}}^{N}-u_{J_{0}}^{0}\right)+\frac{\alpha}{2}\left[\left(u_{J_{N}}^{N}\right)^{2}-\left(u_{J_{0}}^{0}\right)^{2}+\sum_{n=1}^{N}\left(u_{J_{n}}^{n}-u_{J_{n-1}}^{n-1}\right)^{2}\right] .
$$

Applying the obtained expression on (2.35), we have

$$
\begin{aligned}
& \sum_{n=1}^{N} k_{n}\left(c_{1} \sum_{j=1}^{J_{n}-1}+c_{2} \sum_{j=J_{n}+1}^{M-1}\right) h\left(u_{j t}^{n}\right)^{2}+\frac{1}{2}\left(a_{1} \sum_{j=0}^{J_{N}-1}+a_{2} \sum_{j=J_{N}}^{M-1}\right) h\left(u_{j x}^{N}\right)^{2} \\
& \quad+\frac{1}{2} \sum_{n=1}^{N} k_{n}^{2}\left(a_{1} \sum_{j=0}^{J_{n}-1}+a_{2} \sum_{j=J_{n}}^{M-1}\right) h\left(u_{j x t}^{n}\right)^{2}+\frac{\alpha}{2}\left[\left(u_{J_{N}}^{N}\right)^{2}+\sum_{n=1}^{N}\left(u_{J_{n}}^{n}-u_{J_{n-1}}^{n-1}\right)^{2}\right] \\
& =\sum_{n=1}^{N} k_{n}\left(c_{1} \sum_{j=1}^{J_{n}-1}+c_{2} \sum_{j=J_{n}+1}^{M-1}\right) h u_{j t}^{n} \gamma_{j}^{n}+\sum_{n=1}^{N} k_{n}\left(a_{1} \sum_{j=1}^{J_{n}-1}+a_{2} \sum_{j=J_{n}}^{M-1}\right) h u_{j x}^{n} \gamma_{j x}^{n} \\
& \quad+\frac{1}{2}\left(a_{1}-a_{2}\right) \sum_{n=1}^{N-1} h\left(u_{J_{n} x}^{n}\right)^{2}-\alpha \sum_{n=1}^{N} h\left(u_{E}-u_{J_{n}}^{n}\right)\left(u_{J_{n-1}}^{n-1}\right)_{x} \\
& \quad+\alpha \sum_{n=1}^{N} k_{n}\left(u_{E}-u_{J_{n}}^{n}\right) \gamma_{J_{n}}^{n}+\alpha u_{E} u_{J_{N}}^{N}+\frac{1}{2}\left(a_{1} \sum_{j=0}^{J_{0}}+a_{2} \sum_{j=J_{0}+1}^{M-1}\right) h \phi_{j x}^{2}+
\end{aligned}
$$




$$
+\frac{\alpha}{2}\left[\left(\phi_{J_{0}}^{0}\right)^{2}-2 u_{E} \phi_{J_{0}}^{0}\right] .
$$

Let's estimate each term on the right hand side of the last equation. To do for the second and third terms, we introduce the notation $|\gamma|$ and $\left|\gamma_{x}\right|$ for the maximum absolute values of $\gamma$ and $\gamma_{x}$ in the concerned region. By the Schwarz inequality, we have

$$
\begin{aligned}
& \left|\sum_{n=1}^{N} k_{n}\left(c_{1} \sum_{j=1}^{J_{n}-1}+c_{2} \sum_{j=J_{n}+1}^{M-1}\right) h u_{j i t}^{n} \gamma_{j}^{n}\right| \\
& \quad<\frac{1}{2}\left(c_{1}+c_{2}\right)|\gamma|^{2} t_{N}+\frac{1}{2} \sum_{n=1}^{N} k_{n}\left(c_{1} \sum_{j=1}^{J_{n}-1}+c_{2} \sum_{j=J_{n}+1}^{M-1}\right) h\left(u_{j i}^{n}\right)^{2}
\end{aligned}
$$

and

$$
\begin{aligned}
& \left|\sum_{n=1}^{N} k_{n}\left(a_{1} \sum_{j=1}^{J_{n}-1}+a_{2} \sum_{j=J_{n}}^{M-1}\right) h u_{j x}^{n} \gamma_{j x}^{n}\right| \\
& \quad<\frac{1}{2 \varepsilon_{1}}\left(a_{1}+a_{2}\right)\left|\gamma_{x}\right|^{2} t_{N}+\frac{\varepsilon_{1}}{2} \sum_{n=1}^{N} k_{n}\left(a_{1} \sum_{j=1}^{J_{n}-1}+a_{2} \sum_{j=J_{n}}^{M-1}\right) h\left(u_{j x}^{n}\right)^{2},
\end{aligned}
$$

where $\varepsilon_{1}$ is a small positive constant which is given definitely later. The fourth sum on the right hand side of $(2.36)$ is estimated as follows: by (2.12) and (2.15),

$$
\begin{aligned}
\mid-\alpha & \sum_{n=1}^{N} h\left(u_{E}-u_{J_{n}}^{n}\right)\left(u_{J_{n-1}}^{n-1}\right)_{x} \mid<\alpha u_{E}\left(\sum_{n=1}^{N-1} h\left|u_{J_{n}}^{n}\right|+\left|\phi_{1}-\phi_{0}\right|\right) \\
& <\alpha u_{E}\left(\frac{\alpha u_{E}}{b} \sum_{n=1}^{N-1} k_{n} u_{J_{n}}^{n}+\left|\phi_{1}-\phi_{0}\right|\right) \\
& <\frac{\left(\alpha u_{E}\right)^{4}}{2 \varepsilon_{2} b^{2}} t_{N-1}+\frac{\varepsilon_{2}}{2} \sum_{n=1}^{N-1} k_{n}\left(u_{J_{n} x}^{n}\right)^{2}+\alpha u_{E}\left|\phi_{1}-\phi_{0}\right|
\end{aligned}
$$

where $\varepsilon_{2}$ is another positive constant which also is given definitely later. Here, we consider the sum

$$
\sum_{n=1}^{N-1} k_{n}\left(u_{J_{n} x}^{n}\right)^{2} .
$$

From (2.9), we have

$$
u_{J_{n}}^{n}=\frac{1}{a_{2}}\left[a_{1} u_{J_{n} \bar{x}}^{n}-\alpha\left(u_{E}-u_{J_{n}}^{n}\right)\right]
$$

and

$$
\left(u_{J_{n}{ }^{x}}^{n}\right)^{2}<2\left(\frac{a_{1}}{a_{2}}\right)^{2}\left(u_{J_{n} \bar{x}}^{n}\right)^{2}+2\left(\frac{\alpha u_{E}}{a_{2}}\right)^{2} .
$$


Further, we expand $u_{J_{n}^{\bar{x}}}^{n}$ as follows:

$$
u_{J_{n} \bar{x}}^{n}=\sum_{j=1}^{J_{n}-1} h u_{j x \bar{x}}^{n}+u_{0 x}^{n}=\frac{c_{1}}{a_{1}} \sum_{j=1}^{J_{n}-1} h u_{j i}^{n}+u_{0 x}^{n}
$$

(see (2.8).) Hence,

$$
\begin{aligned}
\left(u_{J_{n} \bar{x}}^{n}\right)^{2} & <2\left[N h\left(\frac{c_{1}}{a_{1}}\right)^{2} \sum_{j=1}^{J_{n}-1} h\left(u_{j \bar{t}}^{n}\right)^{2}+\left(u_{0 x}^{n}\right)^{2}\right] \\
& <2\left[\frac{\alpha u_{E} t_{N}}{b}\left(\frac{c_{1}}{a_{1}}\right)^{2} \sum_{j=1}^{J_{n}-1} h\left(u_{j \bar{t}}^{n}\right)^{2}+\left(u_{0 x}^{n}\right)^{2}\right]
\end{aligned}
$$

by (2.15). Applying this inequality on (2.40), we get

$$
\left(u_{J_{n}}^{n}\right)^{2}<4\left[\frac{\alpha u_{E} t_{N}}{b}\left(\frac{c_{1}}{a_{2}}\right)^{2} \sum_{j=1}^{J_{n}-1} h\left(u_{j \bar{t}}^{n}\right)^{2}+\left(\frac{a_{1}}{a_{2}}\right)^{2}\left(u_{0 x}^{n}\right)^{2}\right]+2\left(\frac{\alpha u_{E}}{a_{2}}\right)^{2}
$$

and further

$$
\text { (2.42) } \begin{gathered}
\sum_{n=1}^{N-1} k_{n}\left(u_{J_{n}}^{n}\right)^{2}<\frac{4 \alpha u_{E} t_{N}}{b}\left(\frac{c_{1}}{a_{2}}\right)^{2} \sum_{n=1}^{N-1} k_{n} \sum_{j=1}^{J_{n}-1} h\left(u_{j \bar{t}}^{n}\right)^{2} \\
+4\left(\frac{a_{1}}{a_{2}}\right)^{2} \sum_{n=1}^{N-1} k_{n}\left(u_{0 x}^{n}\right)^{2}+2\left(\frac{\alpha u_{E}}{a_{2}}\right)^{2} t_{N} .
\end{gathered}
$$

Applying this estimation upon (2.39), we have

$$
\text { (2.43) } \begin{aligned}
\mid-\alpha & \sum_{n=1}^{N} h\left(u_{E}-u_{J_{n}}^{n}\right)\left(u_{J_{n-1}}^{n-1}\right)_{x} \mid \\
& <\frac{2 \varepsilon_{2} \alpha u_{E} t_{N}}{b}\left(\frac{c_{1}}{a_{2}}\right)^{2} \sum_{n=1}^{N-1} k_{n} \sum_{j=1}^{J_{n}-1} h\left(u_{j \bar{t}}^{n}\right)^{2}+2 \varepsilon_{2}\left(\frac{a_{1}}{a_{2}}\right)^{2} \sum_{n=1}^{N-1} k_{n}\left(u_{0 x}^{n}\right)^{2} \\
& +\varepsilon_{2}\left(\frac{\alpha u_{E}}{a_{2}}\right)^{2} t_{N}+\frac{\left(\alpha u_{E}\right)^{4}}{2 \varepsilon_{2} b^{2}} t_{N-1}+\alpha u_{E}\left|\phi_{1}-\phi_{0}\right| .
\end{aligned}
$$

Using (2.42) and (2. 15), we also have an estimate for the third sum on the right hand side of (2.36):

$$
\begin{gathered}
\left|\frac{1}{2}\left(a_{1}-a_{2}\right) \sum_{n=1}^{N-1} h\left(u_{J_{n}}^{n}\right)^{2}\right|<2 t_{N}\left(a_{1}-a_{2}\right)\left(\frac{\alpha u_{E} c_{1}}{a_{2} b}\right)^{2} \sum_{n=1}^{N-1} k_{n} \sum_{j=1}^{J_{n}-1} h\left(u_{j i}^{n}\right)^{2} \\
+\frac{2\left(a_{1}-a_{2}\right) \alpha u_{E}}{b}\left(\frac{a_{1}}{a_{2}}\right)^{2} \sum_{n=1}^{N-1} k_{n}\left(u_{0 x}^{n}\right)^{2}+\frac{\left(a_{1}-a_{2}\right)}{a_{2}^{2} b}\left(\alpha u_{E}\right)^{3} t_{N} .
\end{gathered}
$$

Finally, the remained two terms are easily estimated as follows:

$$
\left|\alpha \sum_{n=1}^{N} k_{n}\left(u_{E}-u_{J_{n}}^{n}\right) \gamma_{J_{n}}^{n}\right|<\alpha|\gamma| u_{E} t_{N}
$$


and

$$
\left|\alpha u_{E} u_{J_{N}}^{N}\right|<\alpha u_{E}^{2}
$$

(see (2.12).)

By applying the obtained inequality (2.37)-(2.39) and (2.43)(2.46) upon the right hand side of (2.36), dropping the last bracket on its left hand side and multiplying by 2 , we have

$$
\begin{array}{r}
\sum_{n=1}^{N} k_{n}\left(d_{1} \sum_{j=1}^{J_{n}-1}+c_{2} \sum_{j=J_{n}+1}^{M-1}\right) h\left(u_{j \bar{n}}^{n}\right)^{2}+\left(a_{1} \sum_{j=0}^{J_{N}-1}+a_{2} \sum_{j=J_{N}}^{M-1}\right) h\left(u_{j x}^{N}\right)^{2} \\
<\varepsilon_{1} \sum_{n=1}^{N} k_{n}\left(a_{1} \sum_{j=1}^{J_{n}-1}+a_{2} \sum_{j=J_{n}}^{M-1}\right) h\left(u_{j x}^{n}\right)^{2}+q \sum_{n=1}^{N-1} k_{n}\left(u_{0 x}^{n}\right)^{2}+K
\end{array}
$$

where

$$
\begin{aligned}
& d_{1}=c_{1}-\frac{4 t_{N} \alpha u_{E}}{b}\left(\frac{c_{1}}{a_{2}}\right)^{2}\left[\varepsilon_{2}+\left(a_{1}-a_{2}\right) \frac{\alpha u_{E}}{b}\right], \\
& q=4\left[\varepsilon_{2}+\frac{\left(a_{1}-a_{2}\right) \alpha u_{E}}{b}\right]\left(\frac{a_{1}}{a_{2}}\right)^{2}
\end{aligned}
$$

and

$$
\begin{gathered}
K=\left(a_{1} \sum_{j=0}^{J_{0}}+a_{2} \sum_{j=J_{0}+1}^{M-1}\right) h \phi_{j x}^{2}+\alpha\left[2 u_{E}\left|\phi_{1}-\phi_{0}\right|+2 u_{E}^{2}+\left(\phi_{J_{0}}^{0}\right)^{2}-2 u_{E} \phi_{J_{0}}^{0}\right] \\
+t_{N}\left[\left(c_{1}+c_{2}\right)|\gamma|^{2}+\frac{a_{1}+a_{2}}{\varepsilon_{1}}\left|\gamma_{x}\right|^{2}+2 \varepsilon_{2}\left(\frac{\alpha u_{E}}{a_{2}}\right)^{2}+\frac{\left(\alpha u_{E}\right)^{4}}{\varepsilon_{2} b^{2}}\right. \\
\left.+2 \alpha|\gamma| u_{E}\right] .
\end{gathered}
$$

Now, we take

$$
T_{1}=\min \left\{T_{0}, \frac{1}{16 c_{1}\left(a_{1}-a_{2}\right)}\left(\frac{a_{2} b}{\alpha u_{E}}\right)^{2}\right\}
$$

and

$$
\varepsilon_{2}=\frac{b a_{2}^{2}}{16 T_{1} \alpha u_{E} c_{1}} .
$$

Then, we have

$$
d_{1}>\frac{c_{1}}{2} \text { for } 0<t_{N}<T_{1} \text {. }
$$

Since (2.47), of course, holds for every $t_{N}, 0<t_{N}<T_{1}$, we obtain, from it,

$$
\text { (2.51) } \quad\left(a_{1} \sum_{j=0}^{J_{n}-1}+a_{2} \sum_{j=J_{n}}^{M-1}\right) h\left(u_{j x}^{n}\right)^{2}
$$




$$
<\varepsilon_{1} \sum_{n=1}^{N} k_{n}\left(a_{1} \sum_{j=1}^{J_{n}-1}+a_{2} \sum_{j=J_{n}}^{M-1}\right) h\left(u_{j x}^{n}\right)^{2}+q \sum_{n=1}^{N-1} k_{n}\left(u_{0 x}^{n}\right)^{2}+K
$$

for $n=1,2, \ldots, N$. By multiplying each inequality by $k_{n}$ and summing up, we get

$$
\begin{aligned}
& \sum_{n=1}^{N} k_{n}\left(a_{1} \sum_{j=0}^{J_{n}-1}+a_{2} \sum_{j=J_{n}}^{M-1}\right) h\left(u_{j x}^{n}\right)^{2} \\
& \quad<\varepsilon_{1} T_{1} \sum_{n=1}^{N} k_{n}\left(a_{1} \sum_{j=1}^{J_{n}-1}+a_{2} \sum_{j=J_{n}}^{M-1}\right) h\left(u_{j x}^{n}\right)^{2}+q T_{1} \sum_{n=1}^{N-1} k_{n}\left(u_{0 x}^{n}\right)^{2}+K T_{1}
\end{aligned}
$$

for $0<t_{N}<T_{1}$. Now, we take

$$
\varepsilon_{1}=\frac{1}{2 T_{1}}
$$

Then, the last inequality becomes

(2.52) $\sum_{n=1}^{N} k_{n}\left(a_{1} \sum_{j=0}^{J_{n}-1}+a_{2} \sum_{j=J_{n}}^{M-1}\right) h\left(u_{j x}^{n}\right)^{2}<2 q T_{1} \sum_{n=1}^{N-1} k_{n}\left(u_{0 x}^{n}\right)^{2}+2 K T_{1}$.

Applying (2.50) and (2.52) on (2.47), we obtain

$$
\begin{aligned}
\sum_{n=1}^{N} k_{n} & \left(\frac{c_{1}}{2} \sum_{j=1}^{J_{n}-1}+c_{2} \sum_{j=J_{n}+1}^{M-1}\right) h\left(u_{j i}^{n}\right)^{2}+\left(a_{1} \sum_{j=0}^{J_{N}-1}+a_{2} \sum_{j=J_{N}}^{M-1}\right) h\left(u_{j x}^{N}\right)^{2} \\
& <2 q \sum_{n=1}^{N-1} k_{n}\left(u_{0 x}^{n}\right)^{2}+2 K
\end{aligned}
$$

for $0<t_{N}<T_{1}$.

We are now at the final stage to complete the proof of Lemma 2.3. Suppose that

$$
u_{J_{n}}^{n}<(1-\mu) u_{E} \text { for } 0 \leqq n \leqq N-1 .
$$

Since $T_{1} \leqq T_{0}, T_{0}$ being given in (2.24), we then have, from Lemma 2. 2

$$
\left|u_{0 x}^{n}\right|<H_{1}
$$

for $0<t_{n}<T_{1}$ and $h<h_{0}$. Hence,

$$
\sum_{n=1}^{N-1} k_{n}\left(u_{0 x}^{n}\right)^{2}<H_{1}^{2} t_{N} \text {. }
$$

This and (2.53) yield, especially,

$$
\sum_{j=0}^{M-1} h\left(u_{j x}^{N}\right)^{2}<K \quad \text { for } 0<t_{N}<T_{1}
$$

with another constant $K$. Let's estimate $u_{J_{N}}^{N}$. Now, 


$$
u_{J_{N}}^{N}=u_{J_{N}}^{N}-u_{0}^{0} \leqq \sum_{j=0}^{J_{N}-1} h\left|u_{j x}^{N}\right|+\sum_{n=1}^{N} k_{n}\left|u_{0 \bar{t}}^{n}\right| \text {. }
$$

By applying Schwartz's inequality, (2.56), (2.16) and the Lipshitz continuity of $f_{1}(t),(2.17)$, on the right hand side, we have

$$
u_{J_{N}}^{N}<\left(\frac{\alpha u_{E} T_{1} K}{b}\right)^{1 / 2}+H T_{1} \text {. }
$$

Clearly, we can take $T_{1}$, if necessary, so small that the last right hand side is less than $(1-\mu) u_{E}$. Then, we have (2.54) for $n=N$, too, as far as $0<t_{N}<T_{1}, T_{1}$ a new constant. And, (2.54) is trivial for $n=0$. These facts allow us through induction to get the desired

$$
u_{J_{n}}^{n}<(1-\mu) u_{E} \text { for } 0<t_{n}<T_{1} \text { 。 }
$$

and (2.56). Then, (2.53) becomes

$$
\begin{array}{r}
\sum_{n=1}^{N} k_{n}\left(\frac{c_{1}}{2} \sum_{j=1}^{J_{n}-1}+c_{2} \sum_{j=J_{n}+1}^{M-1}\right) h\left(u_{j \bar{t}}^{n}\right)^{2}+\left(a_{1} \sum_{j=0}^{J_{N}-1}+a_{2} \sum_{j=J_{N}}^{M-1}\right) h\left(u_{j x}^{N}\right)^{2}<K \\
\left(0<t_{N}<T_{1}\right),
\end{array}
$$

with another constant $K$. This certainly produces the desired inequality (2.28). Finally, we also obtain (2.30) from (2.29): by (2.7),

$$
\frac{\max k_{p}}{\min k_{p}}=\frac{u_{E}-\min u_{J_{p-1}^{p}-1}^{p}}{u_{E}-\max u_{J_{p-1}}^{p-1}}<\frac{1}{\mu} .
$$

Thus, we have proved Lemma 2.3 completely.

\section{§ 3. Existence of a Local Solution}

3. 1. In the present section, we will show existence of a local solution of the problem $(1.7)-(1.13)$ by the difference method in the last section. We consider a sequence of $h$ 's tending to 0 . In order to make dependency on $h$ clear, we will use the notation $u_{h j}^{n}$ for $u_{j}^{n}$. We further define an interpolated continuous function $u_{h}(x, t)$ by the formula

$$
\begin{array}{r}
u_{h}(x, t)=u_{h j}^{n}+\left(u_{h j}^{n}\right)_{x}\left(x-x_{j}\right)+\left(u_{h j}^{n}\right)_{t}\left(t-t_{n}\right)+\left(u_{h j}^{n}\right)_{x t}\left(x-x_{j}\right)\left(t-t_{n}\right) \\
\text { in every square }\left\{x_{j} \leqq x<x_{j+1}, t_{n} \leqq t<t_{n+1}\right\},
\end{array}
$$

for all $j$ and $n$. Clearly, the function $u_{h}(x, t)$ has the generalized derivative of the first order, $\frac{\partial u_{h}}{\partial x}$. 
Put again the assumption of Lemma 2.3. Then, from the Lemma

$$
\sum_{n=1}^{N} k_{n} \sum_{j=0}^{M-1} h\left\{\left(u_{h j}^{n}\right)^{2}+\left(u_{h j x}^{n}\right)^{2}+\left(u_{h j}^{n}\right)^{2}\right\}<K \quad\left(t_{N}<T_{1}\right),
$$

where $K$ is a constant not depending on $h$. Here and later, we frequently use the same symbol $K$ for some different constants without notice. The last inequality immediately produces

$$
\int_{0}^{T_{1}} \int_{0}^{1}\left\{\left(u_{h}\right)^{2}+\left(\frac{\partial u_{h}}{\partial x}\right)^{2}+\left(\frac{\partial u_{h}}{\partial t}\right)^{2}\right\} d x d t<K .
$$

In fact, $\left\{u_{h}\right\}$ are bounded uniformly with respect to $h\left(<h_{0}\right)$, and

$$
\begin{aligned}
& \int_{t_{n}}^{t_{n+1}} \int_{x_{j}}^{x_{j+1}}\left\{\left(\frac{\partial u_{h}}{\partial x}\right)^{2}+\left(\frac{\partial u_{h}}{\partial t}\right)^{2}\right\} d x d t \\
& \quad \leqq 2 \int_{t_{n}}^{t_{n+1}} \int_{x_{j}}^{x_{j+1}}\left\{\left(u_{h j x}^{n}\right)^{2}+\left(u_{h j x t}^{n}\right)^{2}\left(t-t_{n}\right)^{2}+\left(u_{h j t}^{n}\right)^{2}+\left(u_{h j x t}^{n}\right)^{2}\left(x-x_{j}\right)^{2}\right\} d x d t \\
& \quad=2\left[\left(u_{h j x}^{n}\right)^{2}+\frac{1}{3}\left(u_{h j x t}^{n}\right)^{2} k_{n+1}^{2}+\left(u_{h j t}^{n}\right)^{2}+\frac{1}{3}\left(u_{h j x t}^{n}\right)^{2} h^{2}\right] k_{n+1} h \\
& \quad \leqq 2\left[\left(u_{h j x}^{n}\right)^{2}+\frac{2}{3}\left\{\left(u_{h j x}^{n+1}\right)^{2}+\left(u_{h j x}^{n}\right)^{2}\right\}+\left(u_{h j t}^{n}\right)^{2}+\frac{2}{3}\left\{\left(u_{h j+1 t}^{n}\right)^{2}+\left(u_{h j t}^{n}\right)^{2}\right\}\right] k_{n+1} h \\
& \quad \leqq \frac{10}{3}\left(u_{h j x}^{n}\right)^{2} h k_{n} \frac{k_{n+1}}{k_{n}}+2\left[\frac{2}{3}\left(u_{h j x}^{n+1}\right)^{2}+\frac{5}{3}\left(u_{h j t}^{n}\right)^{2}+\frac{2}{3}\left(u_{h j+1 t}^{n}\right)^{2}\right] h k_{n+1}
\end{aligned}
$$

and

$$
\frac{k_{n+1}}{k_{n}}=\frac{u_{E}-u_{J_{n-1}}^{n-1}}{u_{E}-u_{J_{n}}^{n}}<\frac{1}{\mu}
$$

by (2.7) and Lemma 2.3. So, we have

$$
\begin{aligned}
\sum_{n=0}^{N-1} & \sum_{j=0}^{M-1} \int_{t_{n}}^{t_{n+1}} \int_{x_{j}}^{x_{j+1}}\left\{\left(\frac{\partial u_{h}}{\partial x}\right)^{2}+\left(\frac{\partial u_{h}}{\partial t}\right)^{2}\right\} d x d t \\
\leqq & \sum_{n=0}^{N-1} \sum_{j=0}^{M-1}\left\{\frac{10}{3 \mu}\left(u_{h j x}^{n}\right)^{2} k_{n} h+2\left[\frac{2}{3}\left(u_{h j x}^{n+1}\right)^{2}+\frac{5}{3}\left(u_{h j i}^{n+1}\right)^{2}\right.\right. \\
& \left.\left.+\frac{2}{3}\left(u_{h j+1 \bar{t}}^{n+1}\right)^{2}\right] k_{n+1} h\right\}
\end{aligned}
$$

and hence obtain (3.3) by (3.2).

Similarly, we again get from Lemma 2.3

$$
\int_{0}^{1}\left\{\left(u_{h}\right)^{2}+\left(\frac{\partial u_{h}}{\partial x}\right)^{2}\right\} d x<K
$$

for every $t\left(0<t<T_{1}\right)$. 
Therefore, we can say that the set of functions $\left\{u_{h}\right\} \quad\left(h<h_{0}\right)$ is contained in a ball in the function space $W_{2}^{1}\left(\Omega_{0}\right)$ (Sobolev space), where $\Omega_{0}$ is the region $\left\{0<x<1,0<t<T_{1}\right\}$, and that $\left\{u_{h}\left({ }^{\circ}, t\right)\right\}\left(h<h_{0}\right)$ is contained in another ball in the space $W_{2}^{1}(0,1)$ for every $t\left(0<t<T_{1}\right)$. From the former fact, we can find a subsequence $\left\{u_{h_{\alpha}}\right\} \quad(\alpha=1,2,3, \ldots)$ such that

a) It converges to a limit function $u \in W_{2}^{1}\left(\Omega_{0}\right)$ weakly in the space $W_{2}^{1}\left(\Omega_{0}\right)$

b) It also converges to the function $u$ strongly in the norm of $L_{2}\left(\Omega_{0}\right)$ and

c) Traces of $u$ are defined almost everywhere on the section $\{0<$ $x<1, t=0\}, \quad\left\{x=0,0<t<T_{1}\right\}$ and $\left\{x=1,0<t<T_{1}\right\}$ respectively, and they are square summable and the following conditions are satisfied at least:

$$
\begin{array}{ll}
\int_{0}^{1}\{u(x, t)-\phi(x)\}^{2} d x \rightarrow 0 & (t \rightarrow 0), \\
\int_{0}^{T_{1}}\left\{u(x, t)-f_{1}(t)\right\}^{2} d t \rightarrow 0 & (x \rightarrow 0), \\
\int_{0}^{T_{1}}\left\{u(x, t)-f_{2}(t)\right\}^{2} d t \rightarrow 0 & (x \rightarrow 1) .
\end{array}
$$

It follows from (b) that there is again a subsequence of $\left\{u_{h_{\alpha}}\right\}$ which converges to $u$ almost everywhere in $\Omega_{0}$. We use again the symbol $\left\{u_{h}\right\}$ for the last sequence.

From the latter fact above, we find that the limit function $u(\circ, t)$ is belongs to $W_{2}^{1}(0,1)$ for any $t\left(0<t<T_{1}\right)$ :

$$
\int_{0}^{1}\left\{u^{2}(x, t)+\left(\frac{\partial u(x, t)}{\partial x}\right)^{2}\right\} d x<K
$$

and hence it is Hörder continuous in $x$ uniformly with respect to $t\left(0<t<T_{1}\right)$ :

$$
\left|u\left(x_{1}, t\right)-u\left(x_{2}, t\right)\right|<K\left|x_{1}-x_{2}\right|^{1 / 2} \quad\left(x_{1}, x_{2} \in[0,1]\right) 。
$$

Further from (3.9), there exist

$$
\lim _{x \rightarrow 0} u(x, t)=f_{1}(t)
$$

and

$$
\lim _{x \rightarrow 1} u(x, t)=f_{2}(t)
$$

for any $t\left(0<t<T_{1}\right)$. 
3. 2. Next, we consider the piecewise linear curve $x=y_{h}(t)$ which connects the point $\left(x_{J_{n}}, t_{n}\right)$ and $\left(x_{J_{n+1}}, t_{n+1}\right)$ for $n=0,1,2, \ldots$ successively. Clearly $y_{h}(t)$ is differentiable almost everywhere in $0<t<T_{1}$ and its derivative $\dot{y}_{h}(t)$ is equal to $-\frac{h}{k_{n}}=\frac{\alpha}{b}\left(u_{E}-u_{J_{n-1}}^{n-1}\right)$ in each interval $\left(t_{n-1}, t_{n}\right) \quad(n=1,2,3, \ldots)$, and again from Lemma 2. 3

$$
\frac{\alpha \mu}{b} u_{E}<\dot{y}_{h}(t) \leqq \frac{\alpha}{b} u_{E} \quad\left(0 \leqq t \leqq T_{1}\right)
$$

So, $\left\{y_{h_{\alpha}}(t)\right\}(\alpha=1,2,3, \ldots)$ constitute a set of uniformly bounded and equicontinuous functions on the interval $\left[0, T_{1}\right]$. Hence, there is a subsequence, for which we again give the symbol $\left\{y_{h_{\alpha}}\right\}$, such that

$$
y_{h_{\alpha}}(t) \rightarrow y(t) \text { uniformly on }\left[0, T_{1}\right],
$$

where $y(t)$ is a Lipshitz continuous function, and satisfies

$$
\frac{\alpha \mu}{b} u_{E}<\frac{y(t)-y(\tau)}{t-\tau} \leqq \frac{\alpha}{b} u_{E} \quad\left(0 \leqq \tau<t \leqq T_{1}\right) .
$$

Finally, it also follows again from (3.9) that

$$
\lim _{x \rightarrow y(t) \pm 0} u(x, t)=u(y(t), t)
$$

exist for every $t\left(0 \leqq t \leqq T_{1}\right)$ and their convergence are uniform in $t$.

3. 3. In order to show that the pair of the function $u$ and $y$ obtained above is a desired solution of our problem (1.7)-(1.13), it remains to prove that

(i) The function $u$ satisfies the equation (1.7) and (1.8), and also the initial condition (1.11), and

(ii) The pair satisfies the internal boundary condition (1.9).

To prove (i), notice that in both regions of

$$
\Omega_{1}=\left\{0<x<y(t), 0<t \leqq T_{1}\right\}
$$

and

$$
\Omega_{2}=\left\{y(t)<x<1,0<t \leqq T_{1}\right\},
$$

the obtained solutions are all uniformly bounded:

$$
0<u_{h j}^{n}<(1-\mu) u_{E} \text {, }
$$

as seen from Lemma 2.3.

It then follows from Theorem A. 7 in Appendix A that $\left\{u_{h x}\right\}$, 
$\left\{u_{h x \bar{x}}\right\}$ and $\left\{u_{h i}\right\}$ are uniformly bounded for all $h\left(<h_{0}\right)$ in any compact set $\Omega_{1}^{*}$ and $\Omega_{2}^{*}$ being contained in $\Omega_{1}$ and $\Omega_{2}$, with a finite distance from the boundary

$$
\partial \Omega_{1}=\left\{x=0,0 \leqq t \leqq T_{1}\right\} \cup\left\{x=y(t), 0 \leqq t \leqq T_{1}\right\}
$$

and

$$
\partial \Omega_{z}=\left\{x=y(t), 0 \leqq t \leqq T_{1}\right\} \cup\left\{x=1,0 \leqq t \leqq T_{1}\right\} \cup\{0 \leqq x \leqq 1, t=0\},
$$

respectively.

Therefore, $\left\{u_{h}(x, t\}(h \rightarrow 0)\right.$ constitute a sequence of functions being uniformly bounded and equicontinuous in $\Omega_{1}^{*}$ and $\Omega_{2}^{*}$, and then allow selection of a subsequence which converges to a continuous function $u(x, t)$ uniformly in both $\Omega_{1}^{*}$ and $\Omega_{2}^{*}$.

Now, we take a sequence of pairs of compact sets $\left\{\Omega_{1 i}^{*}, \Omega_{2 i}^{*}\right\}$ as mentioned above, such that

$$
\Omega_{1 i}^{*} \subset \Omega_{1 i+1}^{*}(i=1,2,3, \ldots), \bigcup_{i=1}^{\infty} \Omega_{1 i}^{*}=\Omega_{1}
$$

and

$$
\Omega_{21}^{*} \subset \Omega_{2 i+1}^{*}(i=1,2,3, \ldots), \bigcup_{i=1}^{\infty} \Omega_{2 i}^{*}=\Omega_{2} \text { 。 }
$$

Take a sequence of subsequences $\left\{u_{h_{i}}\right\}$ with each subsequence $\left\{u_{h_{i}}\right\}$ $(j=1,2, \ldots)$ being taken from its preceding sequence $\left\{u_{h_{l-1} j}\right\} \quad(j=$ $1,2, \ldots)$, and convergent in both $\Omega_{1 i}^{*}$ and $\Omega_{2 i}^{*}$. Make then the sequence of 'diagonal' elements $\left\{u_{n_{i}}\right\}(i=1,2, \ldots)$, as usual. It is easily found that the last sequence converges to a continuous function $u(x, t)$ in both $\Omega_{1}$ and $\Omega_{2}$, and moreover uniformly in any compact set contained in $\Omega_{1}$ or $\Omega_{2}$ 。

We will show that the limit function $u(x, t)$ is not but a solution of the equation of (1.7) and (1.8). According to Theorem A.7, every difference quotient of higher order in $x, t$ is bounded in any compact set contained in $\Omega_{1}$ or $\Omega_{2}$. Therefore, by the same discussion as for $\left\{u_{h}\right\}$ itself, we can further select such a subsequence of the last sequence that not only $\left\{u_{h_{\alpha}}\right\}$, but also $\left\{u_{h_{\alpha} \alpha}\right\}, \quad\left\{u_{h_{\alpha} x \bar{x}}\right\}$ and $\left\{u_{h_{\alpha}}\right\}$ converge uniformly in any compact set in $\Omega_{1}$ or $\Omega_{2}$ to the limit $u(x, t)$ and some continuous function $\bar{u}(x, t), \overline{\bar{u}}(x, t)$ and $\tilde{u}(x, t)$, respectively.

By tending $h_{\alpha}$ to 0 along the selected sequence in the difference 
equation (2.8) and (2.10), we obtain the equation

$$
c_{1} \tilde{u}=a_{1} \overline{\bar{u}} \quad \text { in } \Omega_{1}
$$

and

$$
c_{2} \tilde{u}=a_{2} \overline{\bar{u}} \quad \text { in } \Omega_{2} \text {. }
$$

On the other hand, the trivial relation

$$
u_{h_{\alpha}}\left(x_{j}, t_{n}\right)=u_{h_{\alpha}}\left(x_{j_{0}}, t_{n}\right)+\sum_{i=j_{0}}^{j-1} h_{\alpha} u_{h_{\alpha} x}\left(x_{i}, t_{n}\right) \quad\left(x_{j_{0}}=a\right)
$$

becomes, through the same limit process, the equation

$$
u(x, t)=u(a, t)+\int_{a}^{x} \bar{u}(\xi, t) d \xi .
$$

We then find that $u$ is differentiable in $x$ and

$$
\frac{\partial u}{\partial x}=\bar{u}
$$

and similarly

$$
\frac{\partial u}{\partial t}=\tilde{u} \quad \text { and } \quad \frac{\partial \bar{u}}{\partial x}=\overline{\bar{u}} \text {. }
$$

The obtained relation (3.15)-(3.18) imply that the limit function $u$ satisfies just the equation (1.7) and (1.8). It also is found that the limit function $u(x, t)$ satisfies the initial condition (1.11). (See, for example, $\S 42$ of the famous book [5] by I. G. Petrowsky). We have thus proved (i).

3.4. Let us go to prove (ii). For it, we will take some steps.

3. 4. 1. Lemma 3.1. The limit function $y(t)$ obtained in 3. 2 is continuously differentiable and satisfies

$$
\dot{y}(t)=\frac{\alpha}{b}\left(u_{E}-u(y(t), t)\right)
$$

and

$$
0<\dot{y}(t)<\frac{\alpha u_{E}}{b}
$$

for $0<t<T_{1}$.

Proof. By using the piecewise linear function $y_{h}(t)$ appeared in 3.2, we have 


$$
y_{h}\left(t_{n}\right)=\frac{\alpha}{b} \sum_{p=1}^{n} k_{p}\left(u_{E}-u_{h j_{p-1}^{p}}^{p-1}\right) .
$$

From convergence of $y_{h}(t)$ and $u_{h}$, we get, by taking $h \rightarrow 0$,

$$
y(t)=\frac{\alpha}{b} \int_{0}^{t}\left(u_{E}-u(y(\tau), \tau)\right) d \tau \text {. }
$$

We hence find that $y(t)$ is differentiable everywhere in $0<t<T_{1}$ and the relation (3.19) holds. From (3.19), we also get (3.20) since $0<u(y(t), t)<u_{E}$.

3.4. 2. Lemma 3.2. The derivative $\frac{\partial u}{\partial x}$, u being obtained in 3.3, has finite limit

$$
\lim _{x \rightarrow 0} \frac{\partial u}{\partial x}(x, t)=\frac{\partial u}{\partial x}(+0, t), \lim _{x \rightarrow 1} \frac{\partial u}{\partial x}(x, t)=\frac{\partial u}{\partial x}(1-0, t)
$$

and

$$
\lim _{x \rightarrow y(t) \pm 0} \frac{\partial u}{\partial x}(x, t)=\frac{\partial u}{\partial x}(y(t) \pm 0, t)
$$

almost everywhere in $0<t<T_{1}$, and those limit functions are contained in $L_{2}\left(0, T_{1}\right)$ :

$$
\int_{0}^{T_{1}}\left|\frac{\partial u}{\partial x}(+0, t)\right|^{2} d t<+\infty, \int_{0}^{T_{1}}\left|\frac{\partial u}{\partial x}(1-0, t)\right|^{2} d t<+\infty
$$

and

$$
\int_{0}^{T_{1}}\left|\frac{\partial u}{\partial x}(y(t) \pm 0, t)\right|^{2} d t<+\infty
$$

Proof. According to the well-known existence theorem of a trace operator, we can get it immediately from the facts that

$$
\int_{0}^{T_{1}} \int_{0}^{1}\left|\frac{\partial^{2} u}{\partial x^{2}}\right|^{2} d x d t<\left(\frac{c_{1}}{a_{1}}\right)^{2} \int_{0}^{T_{1}} \int_{0}^{1}\left|\frac{\partial u}{\partial t}\right|^{2} d x d t<+\infty
$$

and $y(t)$ is differentiable and monotone increasing, as shown in Lemma 3.1.

3.4. 3. Lemma 3. 3. The condition

$$
\begin{aligned}
\dot{y}(t) & =\frac{1}{b}\left(a_{1} \frac{\partial u}{\partial x}(y(t)-0, t)-a_{2} \frac{\partial u}{\partial x}(y(t)+0, t)\right) \\
& =\frac{\alpha}{b}\left(u_{E}-u(y(t), t)\right) \quad\left(0<t<T_{1}\right)
\end{aligned}
$$


is satisfied by the pair of function, $\{y(t), u(x, t)\}$ obtained in 3.3.

Proof. Returning to construction of the concerned functions, we again have the relation

$$
y_{h}(t)-y_{h}(\bar{t})=\frac{1}{b} \sum_{p=m+1}^{n} k_{p}\left(a_{1} u_{h_{p^{x}} \bar{x}}-a_{2} u_{h_{p^{x}}}^{p}\right),
$$

where $t=t_{n}$ and $\bar{t}=t_{m}$. According to uniform convergence of $y_{h}(t)$, we can find a positive constant $h_{0}$ for any given constant $\delta>0$ such that

$$
y(t)-\delta<y_{h}(t)<y(t)+\delta \quad\left(\bar{t}<t<T_{1}\right)
$$

for all $h<h_{0}$.

Due to the estimation

$$
\sum_{n=1}^{N} k_{n} \sum_{j=1}^{M-1} h\left(u_{h x \bar{x}}^{n}\right)^{2}<K
$$

we have

$$
\begin{gathered}
\left|\sum_{p=m+1}^{n} k_{p}\left\{u_{h \bar{x}}\left(y\left(t_{p}\right) \pm \delta, t_{p}\right)-u_{h \bar{x}}\left(y_{h}\left(t_{p}\right), t_{p}\right)\right\}\right| \\
<(t-\bar{t})^{1 / 2} \delta^{1 / 2}\left\{\sum_{p=m+1}^{n} k_{p} \sum_{j=1}^{M-1} h\left(u_{j x \bar{x}}^{p}\right)^{2}\right\}^{1 / 2} \\
<K \delta^{1 / 2}
\end{gathered}
$$

for $0<\bar{t}<t<T_{1}$.

From (3.23) and (3.24), we obtain

$$
\begin{aligned}
& \left|y_{h}(t)-y_{h}(\bar{t})-\frac{1}{b} \sum_{p=m+1}^{n} k_{p}\left\{a_{1} u_{h \bar{x}}\left(y\left(t_{p}\right)-\delta, t_{p}\right)-a_{2} u_{h x}\left(y\left(t_{p}\right)+\delta, t_{p}\right)\right\}\right| \\
& \quad<K \delta^{1 / 2} .
\end{aligned}
$$

We take here $h \rightarrow 0$. Then

$$
\left|y(t)-y(\bar{t})-\frac{1}{b} \int_{t}^{t}\left\{a_{1} \frac{\partial u}{\partial x}(y(\tau)-\delta, \tau)-a_{2} \frac{\partial u}{\partial x}(y(\tau)+\delta, \tau)\right\} d \tau\right|<K \delta^{1 / 2} .
$$

Lemma 3.2 here allows to take $\delta \rightarrow 0$ and to get

$$
y(t)-y(\bar{t})=\frac{1}{b} \int_{i}^{t}\left\{a_{1} \frac{\partial u}{\partial x}(y(\tau)-0, \tau)-a_{2} \frac{\partial u}{\partial x}(y(\tau)+0, \tau)\right\} d \tau .
$$

Since $\bar{t}$ is arbitrary, we also get

$$
y(t)=\frac{1}{b} \int_{0}^{t}\left\{a_{1} \frac{\partial u}{\partial x}(y(\tau)-0, \tau)-a_{2} \frac{\partial u}{\partial x}(y(\tau)+0, \tau)\right\} d \tau .
$$


As shown in Lemma $3.1, y(t)$ is continuously differentiable and satisfies (3.19). Therefore, we find that

$$
a_{1} \frac{\partial u}{\partial x}(y(t)-0, t)-a_{2} \frac{\partial u}{\partial x}(y(t)+0, t)
$$

also is continuous and (3.22) follows.

3. 5. In order to show that the obtained pair $\{y(t), u(x, t)\}$ is a classical solution, we must have continuity of not only the expression (3.25) but also $\frac{\partial u}{\partial x}(y(t) \pm 0, t)$ themselves. For the purpose, let us first give an expression for $u(y(t), t)$.

3.5.1. As well known, a function $u(x, t)$ satisfying (1.7) and (1.8) in the respective region can be expressed by using Green's functions:

$$
\begin{aligned}
u(x, t)= & \int_{0}^{t} g_{1}(x, t ; y(\tau), \tau) u(y(\tau), \tau) \dot{y}(\tau) d \tau \\
& +\frac{a_{1}}{c_{1}} \int_{0}^{t} \frac{\partial g_{1}}{\partial \xi}(x, t ; 0, \tau) f_{1}(\tau) d \tau \\
& +\frac{a_{1}}{c_{1}} \int_{0}^{t}\left\{q_{1}(x, t ; y(\tau), \tau) \frac{\partial u}{\partial x}(y(\tau)-0, \tau)\right. \\
& \left.-\frac{\partial g_{1}}{\partial \xi}(x, t ; y(\tau), \tau) u(y(\tau), \tau)\right\} d \tau
\end{aligned}
$$

$$
\left(0<x<y(t), 0<t<T_{1}\right)
$$

and

$$
\begin{aligned}
u(x, t)= & \int_{0}^{1} g_{2}(x, t ; \xi, 0) \phi(\xi) d \xi \\
& -\int_{0}^{t} g_{2}(x, t ; y(\tau), \tau) u(y(\tau), \tau) \dot{y}(\tau) d \tau \\
& -\frac{a_{2}}{c_{2}} \int_{0}^{t} \frac{\partial g_{2}}{\partial \xi}(x, t ; 1, \tau) f_{2}(\tau) d \tau \\
& -\frac{a_{2}}{c_{2}} \int_{0}^{t}\left[g_{2}(x, t ; y(\tau), \tau) \frac{\partial u}{\partial x}(y(\tau)+0, \tau)\right. \\
& \left.-\frac{\partial g_{2}}{\partial \xi}(x, t ; y(\tau), \tau) u(y(\tau), \tau)\right] d \tau \\
\quad & \quad\left(y(t)<x<1,0<t<T_{1}\right)
\end{aligned}
$$

where

$$
\begin{aligned}
& g_{1}(x, t ; \xi, \tau)=U_{1}(x-\xi, t-\tau)-U_{1}(x+\xi, t-\tau), \\
& g_{2}(x, t ; \xi, \tau)=U_{2}(x-\xi, t-\tau)-U_{2}(x+\xi-2, t-\tau)
\end{aligned}
$$

and 


$$
U_{i}(x-\xi, t-\tau)=\frac{\sqrt{c_{i}}}{2 \sqrt{\pi a_{i}(t-\tau)}} \exp \left(-\frac{c_{i}(x-\xi)^{2}}{4 a_{i}(t-\tau)}\right) \quad(i=1,2) .
$$

3.5. 2. We now make an expression for $\sqrt{a_{1} c_{1} u}(y(t)-\delta, t)+\sqrt{a a_{2} c_{2}}(y(t)$ $+\delta, t$ ) from (3.26) and (3.27) with $\delta$ is a positive constant:

$$
\sqrt{a_{1} c_{1}} u(y(t)-\delta, t)+\sqrt{a_{2} c_{2}} u(y(t)+\delta, t)=I_{1}+I_{2}+I_{3}+I_{4}+I_{5}+I_{6}
$$

where

$$
\begin{aligned}
I_{1}(\delta)= & \sqrt{a_{2} c_{2}} \int_{0}^{1} g_{2}(y(t)+\delta, t ; \xi, 0) \phi(\xi) d \xi \\
I_{2}(\delta)= & \sqrt{a_{1} c_{1}} \int_{0}^{t} g_{1}(y(t)-\delta, t ; y(\tau), \tau) u(y(\tau), \tau) \dot{y}(\tau) d \tau \\
& -\sqrt{a_{2} c_{2}} \int_{0}^{t} g_{2}(y(t)+\delta, t ; y(\tau), \tau) u(y(\tau), \tau) \dot{y}(\tau) d \tau, \\
I_{3}(\delta)= & \int_{0}^{t}\left[a_{1}\left(\frac{a_{1}}{c_{1}}\right)^{1 / 2} \frac{\partial g_{1}}{\partial \xi}(y(t)-\delta, t ; 0, \tau) f_{1}(\tau)\right. \\
& \left.-a_{2}\left(\frac{a_{2}}{c_{2}}\right)^{1 / 2} \frac{\partial g_{2}}{\partial \xi}(y(t)+\delta, t ; 1, \tau) f_{2}(\tau)\right] d \tau, \\
I_{4}(\delta)= & \int_{0}^{t}\left[a_{1}\left(\frac{a_{1}}{c_{1}}\right)^{1 / 2} g_{1}(y(t)-\delta, t ; y(\tau), \tau) \frac{\partial u}{\partial x}(y(\tau)-0, \tau)\right. \\
& \left.-a_{2}\left(\frac{a_{2}}{c_{2}}\right)^{1 / 2} g_{2}(y(t)+\delta, t ; y(\tau), \tau) \frac{\partial u}{\partial x}(y(\tau)+0, \tau)\right] d \tau, \\
I_{5}(\delta)= & -a_{1}\left(\frac{a_{1}}{c_{1}}\right)^{1 / 2} \int_{0}^{t} \frac{\partial g_{1}}{\partial \xi}(y(t)-\delta, t ; y(\tau), \tau) u(y(\tau), \tau) d \tau
\end{aligned}
$$

and

$$
I_{6}(\delta)=a_{2}\left(\frac{a_{2}}{c_{2}}\right)^{1 / 2} \int_{0}^{t} \frac{\partial g_{2}}{\partial \xi}(y(t)+\delta, t ; y(\tau), \tau) u(y(\tau), \tau) d \tau .
$$

3. 5. 3. Next, we take $\delta \rightarrow 0$. Since $I_{1}, I_{2}$ and $I_{3}$ depend continuously on $\delta$, we have

$$
\lim _{\delta \rightarrow 0}\left(I_{1}+I_{2}+I_{3}\right)=I_{1}(0)+I_{2}(0)+I_{3}(0) .
$$

Consider $I_{4}$. Its integrand can be expanded as follows:

$$
\begin{gathered}
a_{1}\left(\frac{a_{1}}{c_{1}}\right)^{1 / 2} g_{1}(y(t)-\delta, t ; y(\tau), \tau) \frac{\partial u}{\partial x}(y(\tau)-0, \tau) \\
-a_{2}\left(\frac{a_{2}}{c_{2}}\right)^{1 / 2} g_{2}(y(t)+\delta, t ; y(\tau), \tau) \frac{\partial u}{\partial x}(y(\tau)+0, \tau) \\
=\frac{1}{2 \sqrt{\pi(t-\tau)}}\left\{a _ { 1 } \left[\exp \left(-\frac{c_{1}(y(t)-\delta-y(\tau))^{2}}{4 a_{1}(t-\tau)}\right)\right.\right.
\end{gathered}
$$




$$
\begin{aligned}
& \left.-\exp \left(-\frac{c_{1}(y(t)-\delta+y(\tau))^{2}}{4 a_{1}(t-\tau)}\right)\right] \frac{\partial u}{\partial x}(y(\tau)-0, \tau) \\
& -a_{2}\left[\exp \left(-\frac{c_{2}(y(t)+\delta-y(\tau))^{2}}{4 a_{2}(t-\tau)}\right)\right. \\
& \left.\left.-\exp \left(-\frac{c_{2}(y(t)+\delta+y(\tau)-2)^{2}}{4 a_{2}(t-\tau)}\right)\right] \frac{\partial u}{\partial x}(y(\tau)+0, \tau)\right\} \\
& =\frac{1}{2 \sqrt{\pi(t-\tau)}}\left\{a_{1} \exp \left(-\frac{c_{1}\left[\delta^{2}-2 \delta(y(t)-y(\tau))\right]}{4 a_{1}(t-\tau)}\right) \frac{\partial u}{\partial x}(y(\tau)-0, \tau)\right. \\
& -a_{2} \exp \left(-\frac{c_{2}\left[\delta^{2}+2 \delta(y(t)-y(\tau))\right]}{4 a_{2}(t-\tau)}\right) \frac{\partial u}{\partial x}(y(\tau)+0, \tau) \\
& -a_{1} \exp \left(-\frac{c_{1}\left[\delta^{2}-2 \delta(y(t)-y(\tau))\right]}{4 a_{1}(t-\tau)}\right) \text {. } \\
& \left(1-\exp \left(-\frac{c_{1}(y(t)-y(\tau))^{2}}{4 a_{1}(t-\tau)}\right)\right) \frac{\partial u}{\partial x}(y(\tau)-0, \tau) \\
& +a_{2} \exp \left(-\frac{c_{2}\left[\delta^{2}+2 \delta(y(t)-y(\tau))\right]}{4 a_{2}(t-\tau)}\right) \text {. } \\
& \left(1-\exp \left(-\frac{c_{2}(y(t)-y(\tau))^{2}}{4 a_{2}(t-\tau)}\right)\right) \frac{\partial u}{\partial x}(y(\tau)+0, \tau) \\
& -a_{1} \exp \left(-\frac{c_{1}(y(t)-\delta+y(\tau))^{2}}{4 a_{1}(t-\tau)}\right) \frac{\partial u}{\partial x}(y(\tau)-0, \tau) \\
& \left.+a_{2} \exp \left(-\frac{c_{2}(y(t)+\delta+y(\tau)-2)^{2}}{4 a_{2}(t-\tau)}\right) \frac{\partial u}{\partial x}(y(\tau)+0, \tau)\right\} \text {. }
\end{aligned}
$$

Notice that, since $y(t)$ is a Lipshitz continuous function (see (3.12)),

$$
0<1-\exp \left(-\frac{c_{i}(y(t)-y(\tau))^{2}}{4 a_{i}(t-\tau)}\right)<K(t-\tau) \quad\left(0<\tau<t<T_{1}, i=1,2\right)
$$

holds, and further

$$
\begin{gathered}
\int_{0}^{t}\left|\frac{1}{\sqrt{t-\tau}}\left[1-\exp \left(-\frac{c_{i}(y(t)-y(\tau))^{2}}{4 a_{i}(t-\tau)}\right)\right] \frac{\partial u}{\partial x}(y(\tau) \pm 0, \tau)\right| d \tau \\
<K\left[\int_{0}^{t}(t-\tau) d \tau\right]^{1 / 2}\left[\int_{0}^{t}\left|\frac{\partial u}{\partial x}(y(\tau) \pm 0, \tau)\right|^{2} d \tau\right]^{1 / 2}<+\infty
\end{gathered}
$$

by Lemma 3.2. Also, by the same Lemma,

$$
\begin{aligned}
& \int_{0}^{t}\left|\frac{1}{\sqrt{t-\tau}} \exp \left(-\frac{c_{1}(y(t)+y(\tau))^{2}}{4 a_{1}(t-\tau)}\right) \frac{\partial u}{\partial x}(y(\tau)-0, \tau)\right| d \tau \\
& \quad<\left[\int_{0}^{t} \frac{1}{t-\tau} \exp \left(-\frac{K t^{2}}{t-\tau}\right) d \tau\right]^{1 / 2}\left[\int_{0}^{t}\left|\frac{\partial u}{\partial x}(y(\tau)-0, \tau)\right|^{2} d \tau\right]^{1 / 2}<+\infty \\
& \int_{0}^{t}\left|\frac{1}{\sqrt{t-\tau}} \exp \left(-\frac{c_{2}(y(t)+y(\tau)-2)^{2}}{4 a_{2}(t-\tau)}\right) \frac{\partial u}{\partial x}(y(\tau)+0, \tau)\right| d \tau<
\end{aligned}
$$




$$
\begin{aligned}
<\left[\int_{0}^{t} \frac{1}{t-\tau} \exp \left(-\frac{K\left(1-y\left(T_{1}\right)\right)^{2}}{t-\tau}\right) d \tau\right]^{1 / 2} \\
\cdot\left[\int_{0}^{t}\left|\frac{\partial u}{\partial x}(y(\tau)+0, \tau)\right|_{\mid}^{2} d \tau\right]^{1 / 2}<+\infty .
\end{aligned}
$$

It is already known in 3.4 that $a_{1} \frac{\partial u}{\partial x}(y(\tau)-0, \tau)-a_{2} \frac{\partial u}{\partial x}(y(\tau)+0, \tau)$ is continuous in $\tau$. Therefore, we can take $\delta \rightarrow 0$ inside of the integral sign of $I_{4}(\delta)$, and then have

$$
\text { (3.30) } \begin{aligned}
& \lim _{\delta \rightarrow 0} I_{4}(\delta)=\int_{0}^{t} \frac{1}{2 \sqrt{\pi(t-\tau)}}\left[a_{1} \frac{\partial u}{\partial x}(y(\tau)-0, \tau)-a_{2} \frac{\partial u}{\partial x}(y(\tau)+0, \tau)\right] d \tau \\
& \quad-a_{1} \int_{0}^{t} \frac{1}{2 \sqrt{\pi(t-\tau)}}\left[1-\exp \left(-\frac{c_{1}(y(t)-y(\tau))^{2}}{4 a_{1}(t-\tau)}\right)\right] \frac{\partial u}{\partial x}(y(\tau)-0, \tau) d \tau \\
&+a_{2} \int_{0}^{t} \frac{1}{2 \sqrt{\pi(t-\tau)}}\left[1-\exp \left(-\frac{c_{2}(y(t)-y(\tau))^{2}}{4 a_{2}(t-\tau)}\right)\right] \frac{\partial u}{\partial x}(y(\tau)+0, \tau) d \tau \\
&-a_{1} \int_{0}^{t} \frac{1}{2 \sqrt{\pi(t-\tau)}} \exp \left(-\frac{c_{1}(y(t)+y(\tau))^{2}}{4 a_{1}(t-\tau)}\right) \frac{\partial u}{\partial x}(y(\tau)-0, \tau) d \tau \\
&+a_{2} \int_{0}^{t} \frac{1}{2 \sqrt{\pi(t-\tau)}} \exp \left(-\frac{c_{2}(y(t)+y(\tau)-2)^{2}}{4 a_{2}(t-\tau)}\right) \frac{\partial u}{\partial x}(y(\tau)+0, \tau) d \tau,
\end{aligned}
$$

or

$$
\begin{gathered}
(3.31) \lim _{\delta \rightarrow 0} I_{4}(\delta)=a_{1}\left(\frac{a_{1}}{c_{1}}\right)^{1 / 2} \int_{0}^{t} g_{1}(y(t), t ; y(\tau), \tau) \frac{\partial u}{\partial x}(y(\tau)-0, \tau) d \tau \\
-a_{2}\left(\frac{a_{2}}{c_{2}}\right)^{1 / 2} \int_{0}^{t} g_{2}(y(t), t ; y(\tau), \tau) \frac{\partial u}{\partial x}(y(\tau)+0, \tau) d \tau .
\end{gathered}
$$

3. 5. 4. Next, we consider $I_{5}$, and start from estimation of the following difference with a fixed parameter $s(0<s<t)$ :

$$
\begin{aligned}
D= & \int_{s}^{t} \frac{\partial g_{1}}{\partial \xi}(y(t)-\delta, t ; y(\tau), \tau) u(y(\tau), \tau) d \tau \\
& -\int_{s}^{t} \frac{\partial g_{1}}{\partial \xi}(y(t), t ; y(\tau), \tau) u(y(\tau), \tau) d \tau .
\end{aligned}
$$

Put

$$
D=D_{1}+D_{2}+D_{3}
$$

where

$$
\begin{aligned}
& D_{1}=u(y(t), t) \int_{s}^{t}\left[\frac{\partial g_{1}}{\partial \xi}(y(t)-\delta, t ; y(\tau), \tau)-\frac{\partial g_{1}}{\partial \xi}(y(t), t ; y(\tau), \tau)\right] d \tau, \\
& D_{2}=-\int_{s}^{t} \frac{\partial g_{1}}{\partial \xi}(y(t)-\delta, t ; y(\tau), \tau)[u(y(t), t)-u(y(\tau), \tau)] d \tau
\end{aligned}
$$


and

$$
D_{3}=\int_{s}^{t} \frac{\partial g_{1}}{\partial \xi}(y(t), t ; y(\tau), \tau)[u(y(t), t)-u(y(\tau), \tau)] d \tau .
$$

Let's expand $D_{1}$ as follows:

$$
\begin{aligned}
& D_{1}=\frac{u(y(t), t)}{4 \sqrt{\pi}}\left(\frac{c_{1}}{a_{1}}\right)^{3 / 2}\left\{-\delta \int_{s}^{t} \frac{1}{(t-\tau)^{3 / 2}}\left[\exp \left(-\frac{c_{1}(y(t)-\delta-y(\tau))^{2}}{4 a_{1}(t-\tau)}\right)\right.\right. \\
&\left.+\exp \left(-\frac{c_{1}(y(t)-\delta+y(\tau))^{2}}{4 a_{1}(t-\tau)}\right)\right] d \tau \\
&+\int_{s}^{t} \frac{y(t)-y(\tau)}{(t-\tau)^{3 / 2}}\left[\exp \left(-\frac{c_{1}(y(t)-\delta-y(\tau))^{2}}{4 a_{1}(t-\tau)}\right)\right. \\
&\left.-\exp \left(-\frac{c_{1}(y(t)-y(\tau))^{2}}{4 a_{1}(t-\tau)}\right)\right] d \tau \\
&+\int_{s}^{t} \frac{y(t)+y(\tau)}{(t-\tau)^{3 / 2}} {\left[\exp \left(-\frac{c_{1}(y(t)-\delta+y(\tau))^{2}}{4 a_{1}(t-\tau)}\right)\right.} \\
&\left.\left.-\exp \left(-\frac{c_{1}(y(t)+y(\tau))^{2}}{4 a_{1}(t-\tau)}\right)\right] d \tau\right\} .
\end{aligned}
$$

Since $y(t)$ is Lipshitz continuous and $y(t)+y(\tau)-\delta>y(t)$ for sufficiently small $\delta(<y(s))$, all the integrals except the first integral

$$
J_{1}=-\delta \int_{s}^{t} \frac{1}{(t-\tau)^{3 / 2}} \exp \left(-\frac{c_{1}(y(t)-\delta-y(\tau))^{2}}{4 a_{1}(t-\tau)}\right) d \tau
$$

are absolutely integrable and have an upper bound independent of $\delta$, and allow to take $\delta \rightarrow 0$ under the integral signs.

In order to consider $J_{1}$, let's compare it with an auxiliary integral

$$
\begin{aligned}
J_{1}^{\prime} & =-\delta \int_{s}^{t} \frac{1}{(t-\tau)^{3 / 2}} \exp \left(-\frac{c_{1} \delta^{2}}{4 a_{1}(t-\tau)}\right) d \tau \\
& =-4\left(\frac{a_{1}}{c_{1}}\right)^{1 / 2} \int_{\frac{\delta}{2}\left(\frac{c_{1}}{a_{1}(t-s)}\right)^{1 / 2}}^{\infty} \exp \left(-\omega^{2}\right) d \omega
\end{aligned}
$$

which itself tends to $-2\left(\frac{a_{1} \pi}{c_{1}}\right)^{1 / 2}$ as $\delta \rightarrow 0$. Now,

$$
\begin{aligned}
J_{1}^{\prime}-J_{1}=-\delta \int_{s}^{t} & \frac{1}{(t-\tau)^{3 / 2}} \exp \left(-\frac{c_{1} \delta^{2}}{4 a_{1}(t-\tau)}\right) \\
& \cdot\left[1-\exp \left(-\frac{c_{1}(y(t)-\delta-y(\tau))^{2}-c_{1} \delta^{2}}{4 a_{1}(t-\tau)}\right)\right] d \tau
\end{aligned}
$$

Since $y(t)>y(\tau)$ for $t>\tau>0$ and $y(t)$ is Lipshitz continuous, we have

$$
0<1-\exp \left(-\frac{c_{1}(y(t)-\delta-y(\tau))^{2}-c_{1} \delta^{2}}{4 a_{1}(t-\tau)}\right)
$$




$$
\begin{aligned}
& <\frac{c_{1}(y(t)-y(\tau))}{4 a_{1}(t-\tau)}|y(t)-y(\tau)-2 \delta| \\
& <K(y(t)-y(\tau)+2 \delta) .
\end{aligned}
$$

Hence,

$$
\begin{aligned}
& \left|J_{1}^{\prime}-J_{1}\right|<K\left[\delta^{2} \int_{s}^{t} \frac{1}{(t-\tau)^{3 / 2}} \exp \left(-\frac{c_{1} \delta^{2}}{4 a_{1}(t-\tau)}\right) d \tau\right. \\
& \left.\quad+\delta \int_{s}^{t} \frac{y(t)-y(\tau)}{(t-\tau)^{3 / 2}} d \tau\right]<K(1+\delta) \int_{s}^{t} \frac{1}{\sqrt{t-\tau}} d \tau<K \sqrt{t-s}
\end{aligned}
$$

for sufficiently small $\delta$. Therefore,

$$
\varlimsup_{\delta \rightarrow 0}\left|D_{1}-\frac{u(y(t), t)}{4 \sqrt{\pi}}\left(\frac{c_{1}}{a_{1}}\right)^{3 / 2} J_{1}^{\prime}\right|<K \sqrt{t-s}
$$

or

$$
\varlimsup_{\delta \rightarrow 0}\left|D_{1}+\frac{1}{2}\left(\frac{c_{1}}{a_{1}}\right) u(y(t), t)\right|<K \sqrt{t-s} .
$$

For $D_{2}$, we use the following inequalities:

$$
\begin{aligned}
& \left|\frac{\partial g_{1}}{\partial \xi}(y(t)-\delta, t ; y(\tau), \tau)\right| \\
& \quad<\frac{1}{4 \sqrt{\pi}}\left(\frac{c_{1}}{a_{1}}\right)^{3 / 2} \frac{1}{(t-\tau)^{3 / 2}}\left[\delta \exp \left(-\frac{c_{1}(y(t)-\delta-y(\tau))^{2}}{4 a_{1}(t-\tau)}\right)\right. \\
& \quad+|y(t)-y(\tau)| \exp \left(-\frac{c_{1}(y(t)-\delta-y(\tau))^{2}}{4 a_{1}(t-\tau)}\right) \\
& \left.\quad+|y(t)-\delta+y(\tau)| \exp \left(-\frac{c_{1}(y(t)-\delta+y(\tau))^{2}}{4 a_{1}(t-\tau)}\right)\right]
\end{aligned}
$$

and

$$
\begin{aligned}
& \frac{1}{4 \sqrt{\pi}}\left(\frac{c_{1}}{a_{1}}\right)^{3 / 2} \frac{|y(t)-\delta+y(\tau)|}{(t-\tau)^{3 / 2}} \exp \left(-\frac{c_{1}(y(t)-\delta+y(\tau))^{2}}{4 a_{1}(t-\tau)}\right) \\
& \quad<\frac{1}{2 \sqrt{\pi}}\left(\frac{c_{1}}{a_{1}}\right)^{3 / 2} \frac{y(t)-\delta}{(t-\tau)^{3 / 2}} \exp \left(-\frac{c_{1}(y(t)-\delta)^{2}}{4 a_{1}(t-\tau)}\right) \\
& =\frac{2 c_{1}}{\sqrt{\pi} a_{1}} \frac{d}{d \tau}\left(\frac{c_{1}(y(t)-\delta)}{2 \sqrt{a_{1}(t-\tau)}}\right) \exp \left(-\frac{c_{1}(y(t)-\delta)^{2}}{4 a_{1}(t-\tau)}\right),
\end{aligned}
$$

for sufficiently small $\delta$. Therefore,

$$
\begin{array}{r}
\left|D_{2}\right|<K\left[\left|J_{1}\right|+\int_{0}^{t} \frac{1}{\sqrt{t-\tau}} d \tau+\int_{0}^{\infty} \exp \left(-z^{2}\right) d z\right] \\
\cdot \sup _{s<\tau<t}|u(y(t), t)-u(y(\tau), \tau)|
\end{array}
$$

Since $\left|J_{1}\right|$ is bounded due to (3.34) and (3.35) and both integrals 
on the right hand side also are bounded, the inequality

$$
\left|D_{2}\right|<K \sup _{s<\tau<t}|u(y(t), t)-u(y(\tau), \tau)|
$$

holds. Similarly, we can get

$$
\left|D_{3}\right|<K \sup _{s<\tau<t}|u(y(t), t)-u(y(\tau), \tau)|
$$

By using (3.36)-(3.38), we obtain

$$
\varlimsup_{\delta \rightarrow 0}\left|D+\frac{c_{1}}{2 a_{1}} u(y(t), t)\right|<K\left[\sqrt{t-s}+\sup _{s<\tau<t}|u(y(t), t)-u(y(\tau), \tau)|\right]
$$

On the other hand, it is easily seen that

(3. 40) $\varlimsup_{\delta \rightarrow 0}\left|\int_{0}^{s}\left[\frac{\partial g_{1}}{\partial \xi}(y(t)-\delta, t ; y(\tau), \tau)-\frac{\partial g_{1}}{\partial \xi}(y(t), t ; y(\tau), \tau)\right] u(y(\tau), \tau) d \tau\right|$ $=0$

for a fixed $s(0<s<t)$. From (3.39) and (3.40),

$$
\begin{gathered}
\varlimsup_{\delta \rightarrow 0} \mid \int_{0}^{t}\left[\frac{\partial g_{1}}{\partial \xi}(y(t)-\delta, t ; y(\tau), \tau)-\frac{\partial g_{1}}{\partial \xi}(y(t), t ; y(\tau), \tau)\right] u(y(\tau), \tau) d \tau \\
\quad+\frac{c_{1}}{2 a_{1}} u(y(t), t) \mid<K\left[\sqrt{t-s}+\sup _{s<\tau<t}|u(y(t), t)-u(y(\tau), \tau)|\right] .
\end{gathered}
$$

Here, we can take $s$ arbitrarily near to $t$, so that the right hand side becomes arbitrarily small because of continuity of $u(y(\tau), \tau)$. Thus, we find

$$
\begin{gathered}
\varlimsup_{\delta \rightarrow 0} \mid \int_{0}^{t}\left[\frac{\partial g_{1}}{\partial \xi}(y(t)-\delta, t ; y(\tau), \tau)-\frac{\partial g_{1}}{\partial \xi}(y(t), t ; y(\tau), \tau)\right] u(y(\tau), \tau) d \tau \\
+\frac{c_{1}}{2 a_{1}} u(y(t), t) \mid=0
\end{gathered}
$$

and hence

$$
\text { (3.41) } \begin{aligned}
& \lim _{\delta \rightarrow 0} I_{5}(\delta)=\lim _{\delta \rightarrow 0}-a_{1}\left(\frac{a_{1}}{c_{1}}\right)^{1 / 2} \int_{0}^{t} \frac{\partial g_{1}}{\partial \xi}(y(t)-\delta, t ; y(\tau), \tau) u(y(\tau), \tau) d \tau \\
& =\frac{1}{2} \sqrt{a_{1} c_{1}} u(y(t), t)-a_{1}\left(\frac{a_{1}}{c_{1}}\right)^{1 / 2} \int_{0}^{t} \frac{\partial g_{1}}{\partial \xi}(y(t), t ; y(\tau), \tau) u(y(\tau), \tau) d \tau .
\end{aligned}
$$

Similarly, we get

$$
\text { (3.42) } \begin{aligned}
& \lim _{\delta \rightarrow 0} I_{6}(\delta) \\
& =\frac{1}{2} \sqrt{a_{2} c_{2}} u(y(t), t)+a_{2}\left(\frac{a_{2}}{c_{2}}\right)^{1 / 2} \int_{0}^{t} \frac{\partial g_{2}}{\partial \xi}(y(t), t ; y(\tau), \tau) u(y(\tau), \tau) d \tau .
\end{aligned}
$$

3.5.5. Consequently, we obtain, from (3.28)-(3.30), (3.41)-(3.42), 
(3.43) $\frac{1}{2}\left(\sqrt{a_{1} c_{1}}+\sqrt{a_{2} c_{2}}\right) u(y(t), t)$

$$
\begin{aligned}
& =\sqrt{a_{2} c_{2}} \int_{0}^{1} g_{2}(y(t), t ; \xi, 0) \phi(\xi) d \xi \\
& +\sqrt{a_{1} c_{1}} \int_{0}^{t} g_{1}(y(t), t ; y(\tau), \tau) u(y(\tau), \tau) \dot{y}(\tau) d \tau \\
& \quad-\sqrt{a_{2} c_{2}} \int_{0}^{t} g_{2}(y(t), t ; y(\tau), \tau) u(y(\tau), \tau) \dot{y}(\tau) d \tau \\
& +\int_{0}^{t}\left[a_{1}\left(\frac{a_{1}}{c_{1}}\right)^{1 / 2} \frac{\partial g_{1}}{\partial \xi}(y(t), t ; 0, \tau) f_{1}(\tau)\right. \\
& \left.\quad-a_{2}\left(\frac{a_{2}}{c_{2}}\right)^{1 / 2} \frac{\partial g_{2}}{\partial \xi}(y(t), t ; 1, \tau) f_{2}(\tau)\right] d \tau \\
& +a_{1}\left(\frac{a_{1}}{c_{1}}\right)^{1 / 2} \int_{0}^{t} g_{1}(y(t), t ; y(\tau), \tau) \frac{\partial u}{\partial x}(y(\tau)-0, \tau) d \tau \\
& \quad-a_{2}\left(\frac{a_{2}}{c_{2}}\right)^{1 / 2} \int_{0}^{t} g_{2}(y(t), t ; y(\tau), \tau) \frac{\partial u}{\partial x}(y(\tau)+0, \tau) d \tau \\
& -a_{1}\left(\frac{a_{1}}{c_{1}}\right)^{1 / 2} \int_{0}^{t} \frac{\partial g_{1}}{\partial \xi}(y(t), t ; y(\tau), \tau) u(y(\tau), \tau) d \tau \\
& \quad+a_{2}\left(\frac{a_{2}}{c_{2}}\right)^{1 / 2} \int_{0}^{t} \frac{\partial g_{2}}{\partial \xi}(y(t), t ; y(\tau), \tau) u(y(\tau), \tau) d \tau .
\end{aligned}
$$

For a latter purpose, we further put (3.43) in the following form:

(3. 44) $\frac{1}{2}\left(\sqrt{a_{1} c_{1}}+\sqrt{a_{2} c_{2}}\right) u(y(t), t)$

$$
\begin{aligned}
= & V_{1}(t)+V_{21}(t)+V_{22}(t)+V_{31}(t)+V_{32}(t)+V_{33}(t)+V_{34}(t) \\
& +V_{41}(t)+V_{42}(t)+V_{51}(t)+V_{52}(t),
\end{aligned}
$$

where

(3. 45) $\quad V_{1}(t)=\sqrt{a_{2} c_{2}} \int_{0}^{1} g_{2}(y(t), t ; \xi, 0) \phi(\xi) d \xi$

$$
\begin{aligned}
& V_{21}(t)=\sqrt{a_{1} c_{1}} \int_{0}^{t} g_{1}(y(t), t ; y(\tau), \tau) u(y(\tau), \tau) \dot{y}(\tau) d \tau, \\
& V_{22}(t)=-\sqrt{a_{2} c_{2}} \int_{0}^{t} g_{2}(y(t), t ; y(\tau), \tau) u(y(\tau), \tau) \dot{y}(\tau) d \tau, \\
& V_{31}(t)=\frac{1}{2 \sqrt{\pi}} \int_{0}^{t} \frac{1}{\sqrt{t-\tau}}\left[a_{1} \frac{\partial u}{\partial x}(y(\tau)-0, \tau)-a_{2} \frac{\partial u}{\partial x}(y(\tau)+0, \tau)\right] d \tau, \\
& V_{32}(t)=-\frac{a_{1}}{2 \sqrt{\pi}} \int_{0}^{t} \frac{1}{\sqrt{t-\tau}} \\
& \cdot\left[1-\exp \left(-\frac{c_{1}(y(t)-y(\tau))^{2}}{4 a_{1}(t-\tau)}\right)\right] \frac{\partial u}{\partial x}(y(\tau)-0, \tau) d \tau,
\end{aligned}
$$




$$
\begin{aligned}
& V_{33}(t)=\frac{a_{2}}{2 \sqrt{\pi}} \int_{0}^{t} \frac{1}{\sqrt{t-\tau}} \cdot \\
& \left.\qquad 1-\exp \left(-\frac{c_{2}(y(t)-y(\tau))^{2}}{4 a_{2}(t-\tau)}\right)\right] \frac{\partial u}{\partial x}(y(\tau)+0, \tau) d \tau \\
& V_{34}(t)=-\frac{a_{1}}{2 \sqrt{\pi}} \int_{0}^{t} \frac{1}{\sqrt{t-\tau}} \exp \left(-\frac{c_{1}(y(t)+y(\tau))^{2}}{4 a_{1}(t-\tau)}\right) \frac{\partial u}{\partial x}(y(\tau)-0, \tau) d \tau \\
& +\frac{a_{2}}{2 \sqrt{\pi}} \int_{0}^{t} \frac{1}{\sqrt{t-\tau}} \exp \left(-\frac{c_{2}(y(t)+y(\tau)-2)^{2}}{4 a_{2}(t-\tau)}\right) \frac{\partial u}{\partial x}(y(\tau)+0, \tau) d \tau \\
& V_{41}(t)=-a_{1}\left(\frac{a_{1}}{c_{1}}\right)^{1 / 2} \int_{0}^{t} \frac{\partial g_{1}}{\partial \xi}(y(t), t ; y(\tau), \tau) u(y(\tau), \tau) d \tau \\
& V_{42}(t)=a_{2}\left(\frac{a_{2}}{c_{2}}\right)^{1 / 2} \int_{0}^{t} \frac{\partial g_{2}}{\partial \xi}(y(t), t ; y(\tau), \tau) u(y(\tau), \tau) d \tau \\
& V_{51}(t)=a_{1}\left(\frac{a_{1}}{c_{1}}\right)^{1 / 2} \int_{0}^{t} \frac{\partial g_{1}}{\partial \xi}(y(t), t ; 0, \tau) f_{1}(\tau) d \tau
\end{aligned}
$$

and

$$
V_{52}(t)=-a_{2}\left(\frac{a_{2}}{c_{2}}\right)^{1 / 2} \int_{0}^{t} \frac{\partial g_{2}}{\partial \xi}(y(t), t ; 1, \tau) f_{2}(\tau) d \tau
$$

3. 6. The last expression of $u(y(t), t)$ is used for proof of the following lemma.

Lemma 3.4. The function $u$ satisfies the inequality

$$
\begin{aligned}
& \left|u\left(y\left(t_{2}\right), t_{2}\right)-u\left(y\left(t_{1}\right), t_{1}\right)\right|<K\left[\left|\log \left(t_{2}-t_{1}\right)\right|^{1 / 2}\left(t_{2}-t_{1}\right)^{3 / 4}\right. \\
& \left.+|\log \bar{t}|^{1 / 2}\left(t_{2}-t_{1}\right)^{3 / 4}+\frac{1}{\bar{t}}\left(t_{2}-t_{1}\right)\left|\log \left(t_{2}-t_{1}\right)\right|+\frac{1}{\bar{t}^{3}}\left(t_{2}-t_{1}\right)\right]
\end{aligned}
$$

for any $t_{1}$ and $t_{2}\left(0<\bar{t}<t_{1}<t_{2}<T_{1}\right)$, where $K$ is a constant depending only upon $\bar{t}$ and data.

Proof. We will investigate every term of the right hand side of (3.44) successively.

3. 6. 1. i) $V_{1}(t)$ is continuously differentiable. In fact

$$
\begin{aligned}
& \dot{V}_{1}(t)=-\frac{c_{2}}{4 \sqrt{\pi}} \int_{0}^{1}\left\{\left[\frac{1}{t^{3 / 2}}+\frac{c_{2}(y(t)-\xi) \dot{y}(t)}{a_{2} t^{3 / 2}}\right.\right. \\
& \left.-\frac{c_{2}(y(t)-\xi)^{2}}{2 a_{2} t^{5 / 2}}\right] \exp \left(-\frac{c_{2}(y(t)-\xi)^{2}}{4 a_{2} t}\right)-\left[\frac{1}{t^{3 / 2}}+\frac{c_{2}(y(t)+\xi-2) \dot{y}(t)}{a_{2} t^{3 / 2}}\right. \\
& \left.\left.-\frac{c_{2}(y(t)+\xi-2)^{2}}{2 a_{2} t^{5 / 2}}\right] \exp \left(-\frac{c_{2}(y(t)+\xi-2)^{2}}{4 a_{2} t}\right)\right\} \phi(\xi) d \xi \text {. }
\end{aligned}
$$


By using

$$
\int_{0}^{\infty} \zeta^{n} \exp \left(-\zeta^{2}\right) d \zeta<+\infty \text { for every integer } n,
$$

we find that

$$
\left|\dot{V}_{1}(t)\right|<\frac{K}{t} \quad(t>0)
$$

and hence

$$
\left|V_{1}\left(t_{2}\right)-V_{1}\left(t_{1}\right)\right|<\frac{K}{\bar{t}}\left(t_{2}-t_{1}\right)
$$

for any $t_{1}$ and $t_{2}\left(0<\bar{t}<t_{1}<t_{2}<T_{1}\right)$.

3. 6. 2. ii) Both $V_{21}$ and $V_{22}$ can be treated in a similar way. So, we consider $V_{21}$ only. It contains the integrals with the form

$$
\bar{V}(t)=\int_{0}^{t} \frac{1}{\sqrt{t-\tau}} \phi(t, \tau) d \tau
$$

where

$$
\phi(t, \tau)=\exp \left(-\frac{c_{1}(y(t) \pm y(\tau))^{2}}{4 a_{1}(t-\tau)}\right) u(y(\tau), \tau) \dot{y}(\tau)
$$

It is easily seen that both $\psi$ and $\frac{\partial \psi}{\partial t}$ are bounded and continuous in $t$ and $\tau$. Now, we have

$$
\begin{aligned}
& \left|\bar{V}\left(t_{2}\right)-\bar{V}\left(t_{1}\right)\right|<\left|\int_{0}^{t_{1}}\left[\frac{\phi\left(t_{2}, \tau\right)}{\sqrt{t_{2}-\tau}}-\frac{\psi\left(t_{1}, \tau\right)}{\sqrt{t_{1}-\tau}}\right] d \tau\right|+\left|\int_{0}^{t_{2}} \frac{\phi\left(t_{2}, \tau\right)}{\sqrt{t_{2}-\tau}} d \tau\right| \\
& \leqq \\
& \quad\left|\int_{0}^{t_{1}} \phi\left(t_{2}, \tau\right)\left(\frac{1}{\sqrt{t_{2}-\tau}}-\frac{1}{\sqrt{t_{1}-\tau}}\right) d \tau\right|+\left|\int_{0}^{t_{1}} \frac{1}{\sqrt{t_{1}-\tau}}\left(\phi\left(t_{2}, \tau\right)-\psi\left(t_{1}, \tau\right)\right) d \tau\right| \\
& \quad+\left|\int_{t_{1}}^{t_{2}} \frac{\phi\left(t_{2}, \tau\right)}{\sqrt{t_{2}-\tau}} d \tau\right| \\
& \leqq 2 \sup _{0<\tau<t_{1}}\left|\psi\left(t_{2}, \tau\right)\right|\left[\sqrt{t_{1}}+\left(\sqrt{t_{2}-t_{1}}-\sqrt{t_{2}}\right)\right] \\
& \quad+2\left(t_{2}-t_{1}\right) \sqrt{t_{1}} \sup _{\operatorname{suc}_{0<\tau<t_{1}}\left|\frac{\partial \psi}{\partial t}(t, \tau)\right|+2 \sqrt{t_{2}-t_{1}} \sup _{t_{1}<\tau<t_{2}}\left|\psi\left(t_{2}, \tau\right)\right|,}
\end{aligned}
$$

and hence

(3. 49) $\left|\bar{V}\left(t_{2}\right)-\bar{V}\left(t_{1}\right)\right|<K \sqrt{t_{2}-t_{1}} \quad\left(0<t_{1}<t_{2}\right)$.

It follows directly from the last inequality that

(3.50) $\left|V_{21}\left(t_{2}\right)-V_{21}\left(t_{1}\right)\right|<K \sqrt{t_{2}-t_{1}} \quad\left(0<t_{1}<t_{2}\right)$. 
Similarly, we get

$$
\left|V_{22}\left(t_{2}\right)-V_{22}\left(t_{1}\right)\right|<K \sqrt{t_{2}-t_{1}} \quad\left(0<t_{1}<t_{2}\right) .
$$

3. 6. 3. iii) Consider $V_{31}(t)$. Put

$$
\phi(\tau)=a_{1} \frac{\partial u}{\partial x}(y(\tau)-0, \tau)-a_{2} \frac{\partial u}{\partial x}(y(\tau)+0, \tau) .
$$

Since $\phi(\tau)$ is bounded and continuous in $0<\tau<T_{1}$ as shown in 3.4, we can again apply the estimation method used for $\bar{V}$ in ii), with $\phi(t, \tau)=\phi(\tau)$. Therefore,

(3.52) $\left|V_{31}\left(t_{2}\right)-V_{31}\left(t_{1}\right)\right|<K \sqrt{t_{2}-t_{1}} \quad\left(0<t_{1}<t_{2}\right)$.

3. 6. 4. iv) $V_{32}(t)$ and $V_{33}(t)$ can be dealt with similarly. So, we will consider $V_{32}(t)$ only. Put

$$
\begin{aligned}
& 1-\exp \left(-\frac{c_{1}(y(t)-y(\tau))^{2}}{4 a_{1}(t-\tau)}\right)=1-e^{-X} \\
& =X\left[1-X \int_{0}^{1} \exp (-\theta X)(1-\theta) d \theta\right]=X Z
\end{aligned}
$$

and

$$
y(t)-y(\tau)=(t-\tau) Y, \quad \frac{\partial u}{\partial x}(y(\tau)-0, \tau)=w(\tau)
$$

where

$$
\begin{aligned}
& X=X(t, \tau)=\frac{c_{1}(y(t)-y(\tau))^{2}}{4 a_{1}(t-\tau)} \\
& Z=Z(t, \tau)=1-X(t, \tau) \int_{0}^{1} \exp (-\theta X(t, \tau))(1-\theta) d \theta
\end{aligned}
$$

and

$$
Y=Y(t, \tau)=\int_{0}^{1} \dot{y}(\tau+\theta(t-\tau)) d \theta
$$

Then, $V_{32}(t)$ can be written as

$$
V_{32}(t)=-\frac{c_{1}}{8 \sqrt{\pi}} \int_{0}^{t} \sqrt{t-\tau} Y(t, \tau)^{2} Z(t, \tau) w(\tau) d \tau
$$

Hence, we have

$$
V_{32}\left(t_{2}\right)-V_{32}\left(t_{1}\right)=-\frac{c_{1}}{8 \sqrt{\pi}}\left[\int_{0}^{t_{2}} \sqrt{t_{2}-\tau} Y\left(t_{2}, \tau\right)^{2} Z\left(t_{2}, \tau\right) w(\tau) d \tau\right.
$$




$$
\begin{aligned}
& \left.\quad-\int_{0}^{t_{1}} \sqrt{t_{1}-\tau} Y\left(t_{1}, \tau\right)^{2} Z\left(t_{1}, \tau\right) w(\tau) d \tau\right] \\
& =-\frac{c_{1}}{8 \sqrt{\pi}}\left[\int_{t_{1}}^{t_{2}} \sqrt{t_{2}-\tau} Y\left(t_{2}, \tau\right)^{2} Z\left(t_{2}, \tau\right) w(\tau) d \tau\right. \\
& +\int_{0}^{t_{1}}\left(\sqrt{t_{2}-\tau}-\sqrt{t_{1}-\tau}\right) Y\left(t_{2}, \tau\right)^{2} Z\left(t_{2}, \tau\right) w(\tau) d \tau \\
& \quad+\int_{0}^{t_{1}} \sqrt{t_{1}-\tau}\left(Y\left(t_{2}, \tau\right)-Y\left(t_{1}, \tau\right)\right)\left(Y\left(t_{2}, \tau\right)\right. \\
& \left.+Y\left(t_{1}, \tau\right)\right) Z\left(t_{2}, \tau\right) w(\tau) d \tau+\int_{0}^{t_{1}} \sqrt{t_{1}-\tau} Y\left(t_{1}, \tau\right)^{2}\left(Z\left(t_{2}, \tau\right)\right. \\
& \\
& \left.\left.-Z\left(t_{1}, \tau\right)\right) w(\tau) d \tau\right] .
\end{aligned}
$$

Note that $Y$ and $Z$ are bounded, and $w(\tau)$ is square-integrable in $\left(0, T_{1}\right)$ as shown in Lemma 3.2. Therefore,

$$
\begin{aligned}
& \int_{t_{1}}^{t_{2}} \sqrt{t_{2}-\tau} Y\left(t_{2}, \tau\right)^{2} Z\left(t_{2}, \tau\right) w(\tau) d \tau\left|<K \int_{t_{1}}^{t_{2}} \sqrt{t_{2}-\tau}\right| w(\tau) \mid d \tau \\
& <K\left[\int_{t_{1}}^{t_{2}}\left(t_{2}-\tau\right) d \tau\right]^{1 / 2}\left[\int_{t_{1}}^{t_{2}} w(\tau)^{2} d \tau\right]^{1 / 2}<K\left(t_{2}-t_{1}\right) .
\end{aligned}
$$

Secondly,

$$
\begin{aligned}
& \int_{0}^{t_{1}}\left(\sqrt{t_{2}-\tau}-\sqrt{t_{1}-\tau}\right) Y\left(t_{2}, \tau\right)^{2} Z\left(t_{2}, \tau\right) w(\tau) d \tau \mid \\
& <K\left(t_{2}-t_{1}\right) \int_{0}^{t_{1}} \frac{w(\tau)}{\sqrt{t_{2}-\tau}+\sqrt{t_{1}-\tau}} d \tau \\
& <K\left(t_{2}-t_{1}\right)\left[\int_{0}^{t_{1}} \frac{d \tau}{t_{2}+t_{1}-2 \tau}\right]^{1 / 2}\left[\int_{0}^{t_{1}} w(\tau)^{2} d \tau\right]^{1 / 2} \\
& <K\left(t_{2}-t_{1}\right)\left[\log \frac{t_{2}+t_{1}}{t_{2}-t_{1}}\right]<K\left(t_{2}-t_{1}\right)^{3 / 4}
\end{aligned}
$$

Thirdly,

$$
\begin{aligned}
& \left|\int_{0}^{t_{1}} \sqrt{t_{1}-\tau}\left(Y\left(t_{2}, \tau\right)-Y\left(t_{1}, \tau\right)\right)\left(Y\left(t_{2}, \tau\right)+Y\left(t_{1}, \tau\right)\right) Z\left(t_{2}, \tau\right) w(\tau) d \tau\right| \\
& \quad<K \int_{0}^{t_{1}}\left|Y\left(t_{2}, \tau\right)-Y\left(t_{1}, \tau\right)\right||w(\tau)| d \tau \\
& \quad<K \int_{0}^{t_{1}}\left[\int_{0}^{1}\left|\dot{y}\left(\tau+\theta\left(t_{2}-\tau\right)-\dot{y}\left(\tau+\theta\left(t_{1}-\tau\right)\right) \mid d \theta\right]\right| w(\tau) \mid d \tau\right. \\
& \quad<K \int_{0}^{1} d \theta \int_{0}^{t_{1}}\left|\dot{y}\left(\tau+\theta\left(t_{2}-\tau\right)\right)-\dot{y}\left(\tau+\theta\left(t_{1}-\tau\right)\right)\right||w(\tau)| d \tau \\
& <K \int_{0}^{1} d \theta\left[\int_{0}^{t_{1}}\left|\dot{y}\left(\tau+\theta\left(t_{2}-\tau\right)\right)-\dot{y}\left(\tau+\theta\left(t_{1}-\tau\right)\right)\right|^{2} d \tau\right]^{1 / 2} \\
& \quad=K \int_{0}^{1} \frac{d \theta}{\sqrt{1-\theta}}\left[\int_{0}^{(1-\theta) t_{1}}\left\{\dot{y}\left(\beta+\theta t_{2}\right)-\dot{y}\left(\beta+\theta t_{1}\right)\right\}^{2} d \beta\right]^{1 / 2} .
\end{aligned}
$$


Notice the relation (3.19) and the inequality

$$
\left\{\int_{0}^{T_{1}}\left[u(y(t+h, t+h)-u(y(t), t)]^{2} d t\right\}^{1 / 2}<K h^{3 / 4},\right.
$$

which follows the fact that $u(x, t) \in W_{2}^{2,1}\left(\Omega_{0}\right)$ (see Appendix B). Then, the concerned expression on the right hand side can be estimated as follows :

$$
<K\left(t_{2}-t_{1}\right)^{3 / 4} \int_{0}^{1} \frac{\theta^{3 / 4}}{\sqrt{1-\theta}} d \theta<K\left(t_{2}-t_{1}\right)^{3 / 4}
$$

Finally, we consider the fourth integral on the right hand side of (3.53). Now,

$$
\begin{aligned}
& \left|Z\left(t_{2}, \tau\right)-Z\left(t_{1}, \tau\right)\right| \\
& \quad=\mid-\left(X\left(t_{2}, \tau\right)-X\left(t_{1}, \tau\right)\right) \int_{0}^{1} \exp \left(-\theta X\left(t_{2}, \tau\right)\right)(1-\theta) d \theta \\
& \left.\quad-X\left(t_{1}, \tau\right)\left\{\int_{0}^{1}\left[\exp \left(-\theta X\left(t_{2}, \tau\right)\right)-\exp \left(-\theta X\left(t_{1}, \tau\right)\right)\right](1-\theta) d \theta\right]\right\} \mid .
\end{aligned}
$$

Since $\frac{\partial X}{\partial t}$ is bounded, we get, by the mean value theorem,

$$
\left|Z\left(t_{2}, \tau\right)-Z\left(t_{1}, \tau\right)\right|<K\left(t_{2}-t_{1}\right)
$$

Hence

$$
\begin{gathered}
\left|\int_{0}^{t_{1}} \sqrt{t_{1}-\tau} Y\left(t_{1}, \tau\right)^{2}\left(Z\left(t_{2}, \tau\right)-Z\left(t_{1}, \tau\right)\right) w(\tau) d \tau\right| \\
\quad<K\left(t_{2}-t_{1}\right) \int_{0}^{t_{1}}|w(\tau)| d \tau<K\left(t_{2}-t_{1}\right) .
\end{gathered}
$$

Consequently, we have from (3.54)-(3.57)

$$
\left|V_{32}\left(t_{2}\right)-V_{32}\left(t_{1}\right)\right|<K\left(t_{2}-t_{1}\right)^{3 / 4} \quad\left(0<t_{1}<t_{2}<T_{1}\right) .
$$

Similarly,

$$
\left|V_{33}\left(t_{2}\right)-V_{33}\left(t_{1}\right)\right|<K\left(t_{2}-t_{1}\right)^{3 / 4} \quad\left(0<t_{1}<t_{2}<T_{1}\right) .
$$

3. 6.5. v) The next step is to consider $V_{34}(t)$. By the fact that there is a constant $\gamma(>0)$ such that for $0<\tau<t<T_{1}$

$$
y(t)+y(\tau)>\gamma t \quad \text { and } \quad 2-y(t)-y(\tau)>\gamma
$$

we find

$$
\left|\frac{d V_{34}(t)}{d t}\right|<K \quad\left(0<t<T_{1}\right)
$$


and hence

$$
\left|V_{34}\left(t_{2}\right)-V_{34}\left(t_{1}\right)\right|<K\left(t_{2}-t_{1}\right) \quad\left(0<t_{1}<t_{2}<T_{1}\right) .
$$

vi) For $V_{41}(t)$, put

$$
V_{41}(t)=V_{411}(t)+V_{412}(t),
$$

where

$$
\begin{gathered}
V_{411}(t)=-\frac{c_{1}}{4 \sqrt{\pi}} \int_{0}^{t} \frac{y(t)-y(\tau)}{(t-\tau)^{3 / 2}} \exp \left(-\frac{c_{1}(y(t)-y(\tau))^{2}}{4 a_{1}(t-\tau)}\right) \\
\cdot u(y(\tau), \tau) d \tau \\
V_{412}(t)=-\frac{c_{1}}{4 \sqrt{\pi}} \int_{0}^{t} \frac{y(t)+y(\tau)}{(t-\tau)^{3 / 2}} \exp \left(-\frac{c_{1}(y(t)+y(\tau))^{2}}{4 a_{1}(t-\tau)}\right) \\
\cdot u(y(\tau), \tau) d \tau
\end{gathered}
$$

$V_{411}(t)$ can be expressed as follows:

$$
V_{411}(t)=\int_{0}^{t} \frac{\phi(t, \tau)}{\sqrt{t-\tau}} d \tau
$$

where

$$
\text { (3. 64) } \begin{aligned}
\phi(t, \tau)=-\frac{c_{1}}{4 \sqrt{\pi}} \exp \left(-\frac{c_{1}(y(t)-y(\tau))^{2}}{4 a_{1}(t-\tau)}\right) u(y(\tau), \tau) \\
\cdot \int_{0}^{1} \dot{y}(\tau+\theta(t-\tau)) d \tau .
\end{aligned}
$$

It has the same form of $\bar{V}(t)$ given in ii). But $\frac{\partial \psi}{\partial t}$ may here not exist, so that we must be content with having the following estimate in the first place:

$$
\begin{aligned}
& \left|V_{411}\left(t_{2}\right)-V_{411}\left(t_{1}\right)\right|<\left|\int_{0}^{t_{1}} \phi\left(t_{1}, \tau\right)\left(\frac{1}{\sqrt{t_{1}-\tau}}-\frac{1}{\sqrt{t_{2}-\tau}}\right) d \tau\right| \\
& \quad+\left|\int_{0}^{t_{1}} \frac{1}{\sqrt{t_{2}-\tau}}\left[\phi\left(t_{2}, \tau\right)-\phi\left(t_{1}, \tau\right)\right] d \tau\right|+\left|\int_{t_{1}}^{t_{2}} \frac{\phi\left(t_{2}, \tau\right)}{\sqrt{t_{2}-\tau}} d \tau\right|
\end{aligned}
$$

The first and third term on the right hand side are bounded from upper by

$$
2 \sup _{0<\tau<t_{1}}\left|\psi\left(t_{1}, \tau\right)\right|\left(\sqrt{\overline{t_{2}-t_{1}}}-\left(\sqrt{t_{2}}-\sqrt{\overline{t_{1}}}\right)\right)
$$

and

$$
2 \sup _{t_{1}<\tau<t_{2}}\left|\psi\left(t_{2}, \tau\right)\right| \sqrt{t_{2}-t_{1}}
$$

respectively. We next examine the second term. 


$$
\begin{gathered}
\psi\left(t_{2}, \tau\right)-\phi\left(t_{1}, \tau\right)=-\frac{c_{1}}{4 \sqrt{\pi}}\left\{\left[\exp \left(-\frac{c_{1}\left(y\left(t_{2}\right)-y(\tau)\right)^{2}}{4 a_{1}\left(t_{2}-\tau\right)}\right)\right.\right. \\
\left.-\exp \left(-\frac{c_{1}\left(y\left(t_{1}\right)-y(\tau)\right)^{2}}{4 a_{1}\left(t_{1}-\tau\right)}\right)\right] \circ \int_{0}^{1} \dot{y}\left(\tau+\theta\left(t_{2}-\tau\right)\right) d \theta \\
+\exp \left(-\frac{c_{1}\left(y\left(t_{1}\right)-y(\tau)\right)^{2}}{4 a_{1}\left(t_{1}-\tau\right)}\right)\left[\int _ { 0 } ^ { 1 } \left(\dot{y}\left(\tau+\theta\left(t_{2}-\tau\right)\right)\right.\right. \\
\left.\left.\left.-\dot{y}\left(\tau+\theta\left(t_{1}-\tau\right)\right)\right) d \theta\right]\right\}
\end{gathered}
$$

and

$$
\begin{aligned}
\mid \exp (- & \left.\frac{c_{1}\left(y\left(t_{2}\right)-y(\tau)\right)^{2}}{4 a_{1}\left(t_{2}-\tau\right)}\right)-\exp \left(-\frac{c_{1}\left(y\left(t_{1}\right)-y(\tau)\right)^{2}}{4 a_{1}\left(t_{1}-\tau\right)}\right) \mid \\
& <\left(t_{2}-t_{1}\right) \sup _{t_{1}<t<t_{2}} \mid\left[-\frac{c_{1}(y(t)-y(\tau))}{2 a_{1}(t-\tau)} \dot{y}(t)\right. \\
& \left.+\frac{c_{1}(y(t)-y(\tau))^{2}}{4 a_{1}(t-\tau)^{2}}\right] \exp \left(-\frac{c_{1}(y(t)-y(\tau))^{2}}{4 a_{1}(t-\tau)^{2}}\right) \\
& <K\left(t_{2}-t_{1}\right) .
\end{aligned}
$$

Hence

$$
\begin{aligned}
& \int_{0}^{t_{1}} \frac{1}{\sqrt{t_{2}-\tau}}\left[\phi\left(t_{2}, \tau\right)-\phi\left(t_{1}, \tau\right)\right] d \tau \mid \\
& <K \sqrt{t_{1}}\left(t_{2}-t_{1}\right)+K \mid \int_{0}^{t_{1}} \frac{d \tau}{\sqrt{t_{2}-\tau}} \int_{0}^{1}\left[\dot{y}\left(\tau+\theta\left(t_{2}-\tau\right)\right)\right. \\
& \left.\quad-\dot{y}\left(\tau+\theta\left(t_{1}-\tau\right)\right)\right] d \theta \mid .
\end{aligned}
$$

Furthermore, the last double integral can be estimated as follows:

$$
\begin{aligned}
& \left|\int_{0}^{t_{1}} \frac{d \tau}{\sqrt{t_{2}-\tau}} \int_{0}^{1}\left[\dot{y}\left(\tau+\theta\left(t_{2}-\tau\right)\right)-\dot{y}\left(\tau+\theta\left(t_{1}-\tau\right)\right)\right] d \theta\right| \\
& \quad=\left|\int_{0}^{1} d \theta \int_{0}^{t_{1}} \frac{1}{\sqrt{t_{2}-\tau}}\left[\dot{y}\left(\tau+\theta\left(t_{2}-\tau\right)\right)-\dot{y}\left(\tau+\theta\left(t_{1}-\tau\right)\right)\right] d \tau\right| \\
& \quad \leqq \int_{0}^{1} d \theta\left[\int_{0}^{t_{1}} \frac{d \tau}{t_{2}-\tau}\right]^{1 / 2}\left[\int_{0}^{t_{1}}\left(\dot{y}\left(\tau+\theta\left(t_{2}-\tau\right)\right)-\dot{y}\left(\tau+\theta\left(t_{1}-\tau\right)\right)\right)^{2} d \tau\right]^{1 / 2} \\
& \quad=\left(\log \frac{t_{2}}{t_{2}-t_{1}}\right)^{1 / 2} \int_{0}^{1} \frac{d \theta}{\sqrt{1-\theta}}\left[\int_{0}^{(1-\theta) t_{1}}\left(\dot{y}\left(\beta+\theta t_{2}\right)-\dot{y}\left(\beta+\theta t_{1}\right)\right)^{2} d \beta\right]^{1 / 2}
\end{aligned}
$$

Let's use the estimation method used at the last step to get (3.56). Then we find that the last expression is less than

$$
\begin{aligned}
& K\left(\log \frac{t_{2}}{t_{2}-t_{1}}\right)^{1 / 2}\left(t_{2}-t_{1}\right)^{3 / 4} \\
& \quad<K\left[\left|\log \left(t_{2}-t_{1}\right)\right|^{1 / 2}\left(t_{2}-t_{1}\right)^{3 / 4}+|\log \bar{t}|^{1 / 2}\left(t_{2}-t_{1}\right)^{3 / 4}\right]
\end{aligned}
$$


for $0<\bar{t}<t_{1}<t_{2}<T_{1}$. Therefore

(3.68) $\quad \int_{0}^{t_{1}} \frac{1}{\sqrt{t_{2}-\tau}}\left[\psi\left(t_{2}, \tau\right)-\psi\left(t_{1}, \tau\right)\right] d \tau \mid$ $<K\left[\left|\log \left(t_{2}-t_{1}\right)\right|^{1 / 2}\left(t_{2}-t_{1}\right)^{3 / 4}+|\log \bar{t}|^{1 / 2}\left(t_{2}-t_{1}\right)^{3 / 4}\right]$.

This and the estimates given before, (3.66), (3.67), for terms on the right hand side of (3.65) admit to get

(3. 69) $\left|V_{411}\left(t_{2}\right)-V_{411}\left(t_{1}\right)\right|<K\left[\sqrt{t_{2}-t_{1}}+|\log \bar{t}|^{1 / 2}\left(t_{2}-t_{1}\right)^{3 / 4}\right]$.

3.6. 6. vi) Consider $V_{412}(t)$, and put

$$
V_{412}(t)=\int_{0}^{t} \frac{\psi(t, \tau)}{\sqrt{t-\tau}} d \tau
$$

where

$$
\psi(t, \tau)=-\frac{c_{1}(y(t)+y(\tau))}{4 \sqrt{\pi}(t-\tau)} \exp \left(-\frac{c_{1}(y(t)+y(\tau))^{2}}{4 a_{1}(t-\tau)}\right) u(y(\tau), \tau) .
$$

Notice that both $\psi$ and $\frac{\partial \psi}{\partial t}$ are continuous and bounded in $t$ and $\tau$ $(\tilde{E}<\tau<t)$ since there is a positive constant $\gamma$ such that $y(t)+y(\tau)>\gamma t$. In fact,

$$
|\psi(t, \tau)|<\frac{K}{\bar{t}}, \quad\left|\frac{\partial \psi}{\partial t}(t, \tau)\right|<\frac{K}{\bar{t}^{3}} .
$$

Repeating the discussion done for $\bar{V}$ in (ii) produces the inequality

$$
\left|V_{412}\left(t_{2}\right)-V_{412}\left(t_{1}\right)\right|<K\left[\frac{\sqrt{t_{2}-t_{1}}}{\bar{t}}+\frac{t_{2}-t_{1}}{\bar{t}^{3}}\right] \text {. }
$$

By combining (3.69) and (3.70), we obtain

$$
\begin{aligned}
\left|V_{41}\left(t_{2}\right)-V_{41}\left(t_{1}\right)\right|<K\left[\frac{\sqrt{t_{2}-t_{1}}}{\bar{t}}\right. & +|\log \bar{t}|^{1 / 2}\left(t_{2}-t_{1}\right)^{3 / 4} \\
& \left.+\frac{t_{2}-t_{1}}{\bar{t}^{3}}\right] \quad\left(\bar{t}<t_{1}<t_{2}\right) .
\end{aligned}
$$

Similarly, we can get

$$
\begin{aligned}
\left|V_{42}\left(t_{2}\right)-V_{42}\left(t_{1}\right)\right|<K\left[\frac{\sqrt{t_{2}-t_{1}}}{\bar{t}}\right. & +|\log \bar{t}|^{1 / 2}\left(t_{2}-t_{1}\right)^{3 / 4} \\
& \left.+\frac{t_{2}-t_{1}}{\bar{t}^{3}}\right] \quad\left(\bar{t}<t_{1}<t_{2}\right) .
\end{aligned}
$$


3.6. 7. vii) For $V_{51}$ and $V_{52}$, we have only to repeat the above method for $V_{412}$. We obtain

$$
\begin{aligned}
& \left|V_{51}\left(t_{2}\right)-V_{51}\left(t_{1}\right)\right|<K\left[\frac{\sqrt{t_{2}-t_{1}}}{\bar{t}}+\frac{t_{2}-t_{1}}{\bar{t}^{3}}\right] \quad\left(\bar{t}<t_{1}<t_{2}\right), \\
& \left|V_{52}\left(t_{2}\right)-V_{52}\left(t_{1}\right)\right|<\frac{K \sqrt{t_{2}-t_{1}}}{\bar{t}} \quad\left(\bar{t}<t_{1}<t_{2}\right) .
\end{aligned}
$$

Here, we have used the fact for $V_{52}$ that the corresponding $\psi$ has the uniformly bounded derivative with respect to $t$ for all $t>0$.

3.6. 8. From (3.47), (3.50), (3.51), (3.52), (3.58), (3.59), (3.61), (3.71), (3.72), (3.73) and (3.44), we have

$$
\begin{aligned}
& \left|u\left(y\left(t_{2}\right), t_{2}\right)-u\left(y\left(t_{1}\right), t_{1}\right)\right| \\
& \quad<K\left[\frac{t_{2}-t_{1}}{\bar{t}}+\sqrt{t_{2}-t_{1}}+\left(t_{2}-t_{1}\right)^{3 / 4}+\left(t_{2}-t_{1}\right)+\right. \\
& \left.\quad+\frac{\sqrt{t_{2}-t_{1}}}{\bar{l}}+|\log \bar{t}|^{1 / 2}\left(t_{2}-t_{1}\right)^{3 / 4}+\frac{t_{2}-t_{1}}{\bar{t}^{3}}\right] \quad\left(\bar{t}<t_{1}<t_{2}\right) .
\end{aligned}
$$

Hence, we have

$$
\begin{aligned}
& \left|u\left(y\left(t_{2}\right), t_{2}\right)-u\left(y\left(t_{1}\right), t_{1}\right)\right| \\
& \quad<K\left[\frac{\sqrt{t_{2}-t_{1}}}{\bar{t}}+|\log \bar{t}|^{1 / 2}\left(t_{2}-t_{1}\right)^{3 / 4}+\frac{t_{2}-t_{1}}{\bar{t}^{3}}\right] \quad\left(\bar{t}<t_{1}<t_{2}\right) .
\end{aligned}
$$

However, this has a distance from the desired inequality (3.51). It is necessary to replace $\left(t_{2}-t_{1}\right)^{1 / 2}$ by $\left(t_{2}-t_{1}\right)^{3 / 4}$. For it, we next use the obtained estimate (3.74).

The concerned term $\left(t_{2}-t_{1}\right)^{1 / 2} / \bar{t}$ was produced from the estimations of the integral of type (3.48) in ii), as well as in iii) and iv).

3.6.9. ii') For $V_{21}$ and $V_{22}$, we again consider

$$
\bar{V}(t)=\int_{0}^{t} \frac{1}{\sqrt{t-\tau}} \psi(t, \tau) d \tau
$$

where

(3. 76) $\phi(t, \tau)=\exp \left(-\frac{c_{1}(y(t) \pm y(\tau))^{2}}{4 a_{1}(t-\tau)}\right) u(y(\tau), \tau) \dot{y}(\tau)$.

Since, by (1.9),

$$
u(y(\tau), \tau) \dot{y}(\tau)=\frac{\alpha}{b} u(y(\tau), \tau)\left(u_{E}-u(y(\tau), \tau)\right),
$$


we have

$$
\begin{aligned}
& \left|u\left(y\left(\tau_{2}\right), \tau_{2}\right) \dot{y}\left(\tau_{2}\right)-u\left(y\left(\tau_{1}\right), \tau_{1}\right) \dot{y}\left(\tau_{1}\right)\right| \\
& \quad<K\left[\frac{\sqrt{\tau_{2}-\tau_{1}}}{\bar{t}}+|\log \bar{t}|^{1 / 2}\left(\tau_{2}-\tau_{1}\right)^{3 / 4}+\frac{\tau_{2}-\tau_{1}}{\bar{t}^{3}}\right] \quad\left(\bar{t}<\tau_{1}<\tau_{2}\right),
\end{aligned}
$$

due to (3.74). So, we also have

(3. 77) $\left|\phi\left(t, \tau_{2}\right)-\phi\left(t, \tau_{1}\right)\right|$

$$
<K\left[\frac{\sqrt{\tau_{2}-\tau_{1}}}{\bar{t}}+|\log \bar{t}|^{1 / 2}\left(\tau_{2}-\tau_{1}\right)^{3 / 4}+\frac{\tau_{2}-\tau_{1}}{\bar{t}^{3}}\right] \quad\left(\bar{t}<\tau_{1}<\tau_{2}\right) .
$$

Put

$$
\bar{V}(t)=\int_{0}^{t} \frac{\psi(t, \tau)-\psi\left(t_{2}, t_{2}\right)}{\sqrt{t-\tau}} d \tau+2 \psi\left(t_{2}, t_{2}\right) \sqrt{t}
$$

Then

$$
\text { (3.78) } \begin{aligned}
\bar{V}( & \left.t_{2}\right)-\bar{V}\left(t_{1}\right)=\int_{0}^{t_{1}}\left[\psi\left(t_{2}, \tau\right)-\psi\left(t_{2}, t_{2}\right)\right]\left[\frac{1}{\sqrt{t_{2}-\tau}}-\frac{1}{\sqrt{t_{1}-\tau}}\right] d \tau \\
& +\int_{0}^{t_{1}} \frac{1}{\sqrt{t_{1}-\tau}}\left[\phi\left(t_{2}, \tau\right)-\psi\left(t_{1}, \tau\right)\right] d \tau \\
& +\int_{t_{1}}^{t_{2}} \frac{\phi\left(t_{2}, \tau\right)-\psi\left(t_{2}, t_{2}\right)}{\sqrt{t_{2}-\tau}} d \tau+2 \psi\left(t_{2}, t_{2}\right) \frac{t_{2}-t_{1}}{\sqrt{t_{2}}+\sqrt{t_{1}}} .
\end{aligned}
$$

To estimate the first integral on the right hand side, we divide the integral interval into two parts of $(0, \bar{t})$ and $\left(\bar{t}, t_{1}\right)$. For the integral on $(0, \bar{t})$, we have

$$
\begin{aligned}
& \left|\int_{0}^{\bar{t}}\left[\psi\left(t_{2}, \tau\right)-\psi\left(t_{2}, t_{2}\right)\right]\left[\frac{1}{\sqrt{t_{2}-\tau}}-\frac{1}{\sqrt{t_{1}-\tau}}\right] d \tau\right| \\
& \leqq 2 \max _{t, \tau}|\phi(t, \tau)| \int_{0}^{\bar{t}}\left[\frac{1}{\sqrt{t_{1}-\tau}}-\frac{1}{\sqrt{t_{2}-\tau}}\right] d \tau \\
& \leqq 4 \max _{t, \tau}|\phi(t, \tau)|\left[\frac{t_{2}-t_{1}}{\sqrt{t_{2}-\bar{t}}+\sqrt{t_{1}-\bar{t}}}-\frac{t_{2}-t_{1}}{\sqrt{t_{2}}+\sqrt{t_{1}}}\right] \\
& \leqq \frac{K}{\sqrt{\bar{t}}}\left(t_{2}-t_{1}\right)
\end{aligned}
$$

for $t_{2}>t_{1}>2 \bar{t}$. For the other integral on $\left(\bar{t}, t_{1}\right)$, we use (3.77). Then

$$
\begin{gathered}
\left|\int_{\bar{t}}^{t_{1}}\left[\psi\left(t_{2}, \tau\right)-\psi\left(t_{2}, t_{2}\right)\right]\left[\frac{1}{\sqrt{t_{2}-\tau}}-\frac{1}{\sqrt{t_{1}-\tau}}\right] d \tau\right| \\
<K\left\{\frac{1}{\bar{t}} \int_{\bar{t}}^{t_{1}} \sqrt{t_{2}-\tau}\left[\frac{1}{\sqrt{t_{1}-\tau}}-\frac{1}{\sqrt{t_{2}-\tau}}\right] d \tau+\right.
\end{gathered}
$$




$$
\begin{aligned}
& +|\log \bar{t}|^{1 / 2} \int_{\bar{t}}^{t_{1}}\left(t_{2}-\tau\right)^{3 / 4}\left[\frac{1}{\sqrt{t_{1}-\tau}}-\frac{1}{\sqrt{t_{2}-\tau}}\right] d \tau \\
& \left.+\frac{1}{\bar{t}^{3}} \int_{t}^{t_{1}}\left(t_{2}-\tau\right)\left[\frac{1}{\sqrt{t_{1}-\tau}}-\frac{1}{\sqrt{t_{2}-\tau}}\right] d \tau\right\} .
\end{aligned}
$$

Here, we consider the integral

$$
\int_{\bar{t}}^{t_{1}}\left(t_{2}-\tau\right)^{\frac{1}{2}+\alpha}\left[\frac{1}{\sqrt{t_{1}-\tau}}-\frac{1}{\sqrt{t_{2}-\tau}}\right] d \tau \quad\left(0 \leqq \alpha \leqq \frac{1}{2}\right) .
$$

Put

$$
\left(\frac{t_{2}-\tau}{t_{1}-\tau}\right)^{1 / 2}=\sigma 。
$$

Then, the above integral is reduced to

$$
2\left(t_{2}-t_{1}\right)^{1+\alpha} \int_{0_{1}}^{\infty} \frac{\sigma^{2 \alpha+1}}{(\sigma+1)^{2+\alpha}} \cdot \frac{1}{(\sigma-1)^{1+\alpha}} d \sigma \quad\left(\sigma_{1}=\left(\frac{t_{2}-\bar{t}}{t_{1}-\bar{t}}\right)^{1 / 2}\right) .
$$

When $\alpha=0$, it is less than

$$
\begin{gathered}
2\left(t_{2}-t_{1}\right) \int_{\sigma_{1}}^{\infty}\left[\frac{1}{\sigma-1}-\frac{1}{\sigma}\right] d \sigma=2\left(t_{2}-t_{1}\right) \log \frac{\sigma_{1}}{\sigma_{1}-1} \\
=\left(t_{2}-t_{1}\right) \log \frac{t_{2}-\bar{t}}{t_{2}-t_{1}} .
\end{gathered}
$$

When $0<\alpha \leqq \frac{1}{2}$, the concerning integral is less than

$$
\begin{gathered}
2\left(t_{2}-t_{1}\right)^{1+\alpha} \int_{\sigma_{1}}^{\infty} \frac{1}{(\sigma-1)^{1+\alpha}} d \sigma=\frac{2}{\alpha}\left(t_{2}-t_{1}\right)^{1+\alpha} \circ \frac{1}{\left(\sigma_{1}-1\right)^{\alpha}} \\
=\frac{2}{\alpha}\left(t_{2}-t_{1}\right)\left[\sqrt{t_{1}-\bar{t}}\left(\overline{\sqrt{t_{2}-}-\bar{t}}+\sqrt{t_{1}-\bar{t}}\right)\right]^{\alpha} .
\end{gathered}
$$

By applying the obtained estimations of those integrals on the right hand side of (3.80), we get

$$
\begin{aligned}
& \left|\int_{\bar{t}}^{t_{1}}\left[\psi\left(t_{2}, \tau\right)-\psi\left(t_{2}, t_{2}\right)\right]\left[\frac{1}{\sqrt{t_{2}-\tau}}-\frac{1}{\sqrt{t_{1}-\tau}}\right] d \tau\right| \\
& <K\left[\frac{1}{\bar{t}}\left(t_{2}-t_{1}\right)\left|\log \left(t_{2}-t_{1}\right)\right|+\frac{1}{\bar{t}^{3}}\left(t_{2}-t_{1}\right)\right] .
\end{aligned}
$$

The second integral on the right hand side of (3.78) is estimated just as before:

$$
\begin{aligned}
& \left|\int_{0}^{t_{1}} \frac{1}{\sqrt{t_{1}-\tau}}\left[\psi\left(t_{2}, \tau\right)-\psi\left(t_{1}, \tau\right)\right] d \tau\right|<2\left(t_{2}-t_{1}\right) \sqrt{t_{1}} \max _{\substack{0 \leqq \tau \leqq t_{1} \\
t_{1} \leqq t \leqq t_{2}}}\left|\frac{\partial \psi}{\partial t}(t, \tau)\right| \\
& \quad<K\left(t_{2}-t_{1}\right) .
\end{aligned}
$$


For the third integral of (3.78), we again use (3.77). So, we have (3. 83)

$$
\begin{aligned}
& \left|\int_{t_{1}}^{t_{2}} \frac{\psi\left(t_{2}, \tau\right)-\psi\left(t_{2}, t_{2}\right)}{\sqrt{t_{2}-\tau}} d \tau\right| \\
& \quad<K \int_{t_{1}}^{t_{2}}\left[\frac{1}{\bar{t}}+|\log \bar{t}|^{1 / 2}\left(t_{2}-\tau\right)^{1 / 4}+\frac{1}{\bar{t}^{3}} \sqrt{t_{2}-\tau}\right] d \tau \\
& \quad=K\left[\frac{1}{\bar{t}}\left(t_{2}-t_{1}\right)+\frac{4}{5}|\log \bar{t}|^{1 / 2}\left(t_{2}-t_{1}\right)^{5 / 4}+\frac{2}{3 \bar{t}^{3}}\left(t_{2}-t_{1}\right)^{3 / 2}\right]
\end{aligned}
$$

for $t_{2}>t_{1}>\bar{t}$.

For the last term of (3.78), we have trivially

$$
2\left|\psi\left(t_{2}, t_{2}\right) \frac{t_{2}-t_{1}}{\sqrt{\bar{t}_{2}}+\sqrt{t_{1}}}\right|<\frac{K}{\sqrt{\bar{t}}}\left(t_{2}-t_{1}\right) \quad \text { for } \bar{t}<t_{1}<t_{2} .
$$

By applying (3.79) - (3.84) on the right hand side of (3.78), we obtain

$$
\left|\bar{V}\left(t_{2}\right)-\bar{V}\left(t_{1}\right)\right|<K\left[\frac{1}{\bar{t}^{3}}\left(t_{2}-t_{1}\right)+\frac{1}{\bar{t}}\left(t_{2}-t_{1}\right)\left|\log \left(t_{2}-t_{1}\right)\right|\right]
$$

for $t_{2}>t_{1}>2 \bar{t}$.

Using (3.85) instead of (3.49) for $V_{21}$ and $V_{22}$, we obtain the revised estimation

$$
\begin{array}{r}
\left|V_{21}\left(t_{2}\right)-V_{21}\left(t_{1}\right)\right|<K\left[\frac{1}{\bar{t}^{3}}\left(t_{2}-t_{1}\right)+\frac{1}{\bar{t}}\left(t_{2}-t_{1}\right)\left|\log \left(t_{2}-t_{1}\right)\right|\right] \\
\left(t_{2}>t_{1}>2 \bar{t}\right)
\end{array}
$$

and

$$
\text { (3.87) }\left|V_{22}\left(t_{2}\right)-V_{22}\left(t_{1}\right)\right|<K\left[\frac{1}{\bar{t}^{3}}\left(t_{2}-t_{1}\right)+\frac{1}{\bar{t}}\left(t_{2}-t_{1}\right)\left|\log \left(t_{2}-t_{1}\right)\right|\right] .
$$

3.6. 10. iii') For $V_{31}$, we must reconsider the integral of type

$$
\bar{V}(t)=\int_{t}^{t} \frac{1}{\sqrt{t-\tau}} \psi(\tau) d \tau
$$

where

$$
\begin{aligned}
\psi(\tau) & =a_{1} \frac{\partial u}{\partial x}(y(\tau)-0, \tau)-a_{2} \frac{\partial u}{\partial x}(y(\tau)+0, \tau) \\
& =\alpha\left(u_{E}-u(y(\tau), \tau)\right) .
\end{aligned}
$$

By using (3.74), we have the estimation of same type as in (3.77): 
(3. 88) $\left|\phi\left(\tau_{2}\right)-\phi\left(\tau_{1}\right)\right|<K\left[\frac{\sqrt{\tau_{2}-\tau_{1}}}{\bar{t}}+|\log \bar{t}|^{1 / 2}\left(\tau_{2}-\tau_{1}\right)^{3 / 4}+\frac{\tau_{2}-\tau_{1}}{\bar{t}^{3}}\right]$.

Hence, we again have (3.85) for the present $\bar{V}(t)$, and further

$$
\left|V_{31}\left(t_{2}\right)-V_{31}\left(t_{1}\right)\right|<K\left[\frac{1}{\bar{t}^{3}}\left(t_{2}-t_{1}\right)+\frac{1}{\bar{t}}\left(t_{2}-t_{1}\right)\left|\log \left(t_{2}-t_{1}\right)\right|\right] \text {. }
$$

3. 6. 11. vi') Also for $V_{411}(t)$, we use the following expression:

$$
\begin{aligned}
& V_{411}\left(t_{2}\right)-V_{411}\left(t_{1}\right)=\int_{0}^{t_{1}}\left[\psi\left(t_{2}, \tau\right)-\psi\left(t_{2}, t_{2}\right)\right]\left[\frac{1}{\sqrt{t_{2}-\tau}}-\frac{1}{\sqrt{t_{1}-\tau}}\right] d \tau \\
& \quad+\int_{0}^{t_{1}}\left[\psi\left(t_{2}, \tau\right)-\psi\left(t_{1}, \tau\right)\right] \frac{1}{\sqrt{t_{2}-\tau}} d \tau \\
& \quad+\int_{0}^{t_{1}}\left[\psi\left(t_{2}, \tau\right)-\psi\left(t_{1}, \tau\right)\right]\left[\frac{1}{\sqrt{t_{1}-\tau}}-\frac{1}{\sqrt{t_{2}-\tau}}\right] d \tau \\
& \quad+\phi\left(t_{2}, t_{2}\right)\left[\int_{0}^{t_{2}} \frac{d \tau}{\sqrt{t_{2}-\tau}}-\int_{0}^{t_{1}} \frac{d \tau}{\sqrt{t_{1}-\tau}}\right]+\int_{t_{1}}^{t_{2}} \frac{\phi\left(t_{2}, \tau\right)-\psi\left(t_{2}, t_{2}\right)}{\sqrt{t_{2}-\tau}} d \tau,
\end{aligned}
$$

where

$$
\phi(t, \tau)=-\frac{c_{1}}{4 \sqrt{\pi}} \exp \left(-\frac{c_{1}(y(t)-y(\tau))^{2}}{4 a_{1}(t-\tau)}\right) u(y(\tau), \tau) \int_{0}^{1} \dot{y}(\tau+\theta(t-\tau)) d \theta .
$$

To estimate the first integral on the right hand side of (3.90), we again devide the interval $\left(0, t_{1}\right)$ into the two parts of $(0, \bar{t})$ and $\left(\vec{t}, t_{1}\right)$. On the first part, we have the same eatimation as in (3.79) with another constant $K$ :

$$
\left|\int_{0}^{\bar{t}}\left[\phi\left(t_{2}, \tau\right)-\psi\left(t_{2}, t_{2}\right)\right]\left[\frac{1}{\sqrt{t_{2}-\tau}}-\frac{1}{\sqrt{t_{1}-\tau}}\right] d \tau\right|<\frac{K}{\sqrt{\bar{t}}}\left(t_{2}-t_{1}\right)
$$

for $t_{2}>t_{1}>2 \bar{t}$. For the second part, we use (3.74). In fact,

$$
\begin{aligned}
& \phi\left(t_{2}, t_{2}\right)-\phi\left(t_{2}, \tau\right)=-\frac{c_{1}}{4 \sqrt{\pi}}\left[u\left(y\left(t_{2}\right), t_{2}\right) \dot{y}\left(t_{2}\right)\right. \\
& \left.\quad-\exp \left(-\frac{c_{1}\left(y\left(t_{2}\right)-y(\tau)\right)^{2}}{4 a_{1}\left(t_{2}-\tau\right)}\right) u(y(\tau), \tau) \int_{0}^{1} \dot{y}\left(\tau+\theta\left(t_{2}-\tau\right)\right) d \theta\right] \\
& =-\frac{c_{1}}{4 \sqrt{\pi}}\left\{\left[1-\exp \left(-\frac{c_{1}\left(y\left(t_{2}\right)-y(\tau)\right)^{2}}{4 a_{1}\left(t_{2}-\tau\right)}\right)\right] u\left(y\left(t_{2}\right), t_{2}\right) \dot{y}\left(t_{2}\right)\right. \\
& \quad+\exp \left(-\frac{c_{1}\left(y\left(t_{2}\right)-y(\tau)\right)^{2}}{4 a_{1}\left(t_{2}-\tau\right)}\right)\left[u\left(y\left(t_{2}\right), t_{2}\right)-u(y(\tau), \tau)\right] \dot{y}\left(t_{2}\right) \\
& \left.\quad+\exp \left(-\frac{c_{1}\left(y\left(t_{2}\right)-y(\tau)\right)^{2}}{4 a_{1}\left(t_{2}-\tau\right)}\right) \int_{0}^{1}\left[\dot{y}\left(t_{2}\right)-\dot{y}\left(\tau+\theta\left(t_{2}-\tau\right)\right)\right] d \theta\right\} .
\end{aligned}
$$

Now, we can use (3.74) since $t_{2}>\tau>\bar{t}$, and then have 


$$
\left|u\left(y\left(t_{2}\right), t_{2}\right)-u(y(\tau), \tau)\right|<K\left[\frac{\sqrt{t_{2}-\tau}}{\bar{t}}+|\log \bar{t}|^{1 / 2}\left(t_{2}-\tau\right)^{3 / 4}+\frac{t_{2}-\tau}{\bar{t}^{3}}\right]
$$

and also by (3.19)

$$
\begin{aligned}
& \left|\dot{y}\left(t_{2}\right)-\dot{y}\left(\tau+\theta\left(t_{2}-\tau\right)\right)\right|=\frac{\alpha}{b} \mid u\left(y\left(t_{2}\right), t_{2}\right) \\
& \quad-u\left(y\left(\tau+\theta\left(t_{2}-\tau\right)\right), \tau+\theta\left(t_{2}-\tau\right)\right) \mid<K\left[\frac{\sqrt{(1-\theta)\left(t_{2}-\tau\right)}}{\bar{t}}\right. \\
& \left.\quad+|\log \bar{t}|^{1 / 2}\left[(1-\theta)\left(t_{2}-\tau\right)\right]^{3 / 4}+\frac{(1-\theta)\left(t_{2}-\tau\right)}{\bar{t}^{3}}\right] .
\end{aligned}
$$

Therefore, we again have

$$
\text { (3.92) }\left|\psi\left(t_{2}, t_{2}\right)-\phi\left(t_{2}, \tau\right)\right|<K\left[\frac{\sqrt{t_{2}-\tau}}{\bar{t}}+|\log \bar{t}|^{1 / 2}\left(t_{2}-\tau\right)^{3 / 4}+\frac{t_{2}-\tau}{\bar{t}^{3}}\right] \text {. }
$$

This is an estimation of the same form as of (3.77). Hence, just as for (3.80), we obtain

$$
\begin{aligned}
& \left|\int_{\bar{t}}^{t_{1}}\left[\psi\left(t_{2}, \tau\right)-\phi\left(t_{2}, t_{2}\right)\right]\left[\frac{1}{\sqrt{t_{2}-\tau}}-\frac{1}{\sqrt{t_{1}-\tau}}\right] d \tau\right| \\
& \quad<K\left[\frac{1}{\bar{t}}\left(t_{2}-t_{1}\right)\left|\log \left(t_{2}-t_{1}\right)\right|+\frac{1}{\bar{t}^{3}}\left(t_{2}-t_{1}\right)\right] .
\end{aligned}
$$

For the second integral on the right hand side of (3.90), we can use (3.68) without change. For the third integral, we consider the difference $\phi\left(t_{2}, \tau\right)-\phi\left(t_{1}, \tau\right)$ :

$$
\begin{gathered}
\phi\left(t_{2}, \tau\right)-\phi\left(t_{1}, \tau\right)=-\frac{c_{1}}{4 \sqrt{\pi}}\left[\exp \left(-\frac{c_{1}\left(y\left(t_{2}\right)-y(\tau)\right)^{2}}{4 a_{1}\left(t_{2}-\tau\right)}\right)\right. \\
\left.-\exp \left(-\frac{c_{1}\left(y\left(t_{1}\right)-y(\tau)\right)^{2}}{4 a_{1}\left(t_{1}-\tau\right)}\right)\right] u(y(\tau), \tau) \int_{0}^{1} \dot{y}\left(\tau+\theta\left(t_{1}-\tau\right)\right) d \theta \\
-\frac{c_{1}}{4 \sqrt{\pi}} \exp \left(-\frac{c_{1}\left(y\left(t_{2}\right)-y(\tau)\right)^{2}}{4 a_{1}\left(t_{2}-\tau\right)}\right) u(y(\tau), \tau) \int_{0}^{1}\left[\dot{y}\left(\tau+\theta\left(t_{2}-\tau\right)\right)\right. \\
\left.-\dot{y}\left(\tau+\theta\left(t_{1}-\tau\right)\right)\right] d \theta .
\end{gathered}
$$

Hence

$$
\begin{aligned}
\left|\psi\left(t_{2}, \tau\right)-\phi\left(t_{1}, \tau\right)\right|<K\left[t_{2}-t_{1}\right. & +\int_{0}^{1} \mid \dot{y}\left(\tau+\theta\left(t_{2}-\tau\right)\right) \\
& \left.-\dot{y}\left(\tau+\theta\left(t_{1}-\tau\right)\right) \mid d \theta\right]
\end{aligned}
$$

and

$$
\left|\int_{0}^{t_{1}}\left[\phi\left(t_{2}, \tau\right)-\phi\left(t_{1}, \tau\right)\right]\left[\frac{1}{\sqrt{t_{1}-\tau}}-\frac{1}{\sqrt{t_{2}-\tau}}\right] d \tau\right|
$$




$$
\begin{aligned}
& <K\left\{\left(t_{2}-t_{1}\right) \int_{0}^{t_{1}}\left[\frac{1}{\sqrt{t_{1}-\tau}}-\frac{1}{\sqrt{t_{2}-\tau}}\right] d \tau\right. \\
& +\int_{0}^{t_{1}}\left[\frac{1}{\sqrt{t_{1}-\tau}}-\frac{1}{\sqrt{t_{2}-\tau}}\right]\left[\int_{0}^{1} \mid \dot{y}\left(\tau+\theta\left(t_{2}-\tau\right)\right)\right. \\
& \left.\left.\quad-\dot{y}\left(\tau+\theta\left(t_{1}-\tau\right)\right) \mid d \theta\right] d \tau\right\} .
\end{aligned}
$$

The integral of the first term on the right hand side can be easily evaluated. For the second term, we devide the interval $\left(0, t_{1}\right)$ into two parts, $(0, \bar{t})$ and $\left(\bar{t}, t_{1}\right)$. For the former part, we have

$$
\begin{gathered}
\int_{0}^{\bar{t}}\left[\frac{1}{\sqrt{t_{1}-\tau}}-\frac{1}{\sqrt{t_{2}-\tau}}\right]\left[\int_{0}^{1}\left|\dot{y}\left(\tau+\theta\left(t_{2}-\tau\right)\right)-\dot{y}\left(\tau+\theta\left(t_{1}-\tau\right)\right)\right| d \theta\right] d \tau \\
<\int_{0}^{1} d \theta \int\left\{\int_{0}^{t}\left(\frac{1}{\sqrt{t_{1}-\tau}}-\frac{1}{\sqrt{t_{2}-\tau}}\right)^{2} d \tau\right\}^{1 / 2}\left\{\int _ { 0 } ^ { \overline { t } } \left[\dot{y}\left(\tau+\theta\left(t_{2}-\tau\right)\right)\right.\right. \\
\left.\left.-\dot{y}\left(\tau+\theta\left(t_{1}-\tau\right)\right)\right]^{2} d \tau\right\}^{1 / 2}
\end{gathered}
$$

Here, we further have

$$
\begin{aligned}
\int_{0}^{\bar{t}}\left(\frac{1}{\sqrt{t_{1}-\tau}}-\frac{1}{\sqrt{t_{2}-\tau}}\right)^{2} d \tau & <\left(t_{2}-t_{1}\right)^{2} \int_{0}^{\bar{t}} \frac{d \tau}{\left(t_{1}-\tau\right)^{3}} \\
& <\frac{K\left(t_{2}-t_{1}\right)^{2}}{\bar{t}^{2}}
\end{aligned}
$$

for $0<2 \bar{t}<t_{1}<t_{2}<T_{1}$, and

$$
\int_{0}^{1} d \theta\left\{\int_{0}^{\bar{t}}\left[\dot{y}\left(\tau+\theta\left(t_{2}-\tau\right)\right)-\dot{y}\left(\tau+\theta\left(t_{1}-\tau\right)\right)\right]^{2} d \tau\right\}^{1 / 2}<K\left(t_{2}-t_{1}\right)^{3 / 4}
$$

by the same method used to get $(3.56)$. Therefore

$$
\begin{aligned}
\int_{0}^{\bar{t}} & {\left[\frac{1}{\sqrt{t_{1}-\tau}}-\frac{1}{\sqrt{t_{2}-\tau}}\right]\left[\int_{0}^{1}\left|\dot{y}\left(\tau+\theta\left(t_{2}-\tau\right)\right)-\dot{y}\left(\tau+\theta\left(t_{1}-\tau\right)\right)\right| d \theta\right] d \tau } \\
& <K\left[\frac{\left(t_{2}-t_{1}\right)^{2}}{\bar{t}^{2}}+\left(t_{2}-t_{1}\right)^{3 / 4}\right] \quad\left(2 \bar{t}<t_{1}<t_{2}\right) .
\end{aligned}
$$

For the latter part, $\bar{t}<\tau<t_{1}$, we again use (3.19) and (3.74):

$$
\begin{aligned}
\left|\dot{y}\left(\tau+\theta\left(t_{2}-\tau\right)\right)-\dot{y}\left(\tau+\theta\left(t_{1}-\tau\right)\right)\right| & \\
= & \frac{\alpha}{b} \mid u\left(y\left(\tau+\theta\left(t_{1}-\tau\right)\right), \tau+\theta\left(t_{1}-\tau\right)\right) \\
& -u\left(y\left(\tau+\theta\left(t_{2}-\tau\right)\right), \tau+\theta\left(t_{2}-\tau\right)\right) \mid \\
& <K\left[\frac{\sqrt{t_{2}-t_{1}}}{\bar{t}}+|\log \bar{t}|^{1 / 2}\left(t_{2}-t_{1}\right)^{3 / 4}+\frac{t_{2}-t_{1}}{\bar{t}^{3}}\right]
\end{aligned}
$$

and 


$$
\begin{aligned}
& \int_{t}^{t_{1}} {\left[\frac{1}{\sqrt{t_{1}-\tau}}-\frac{1}{\sqrt{t_{2}-\tau}}\right] d \tau=\left(t_{2}-t_{1}\right) \int_{\bar{t}}^{t_{1}} \frac{d \tau}{\sqrt{t_{1}-\tau} \sqrt{t_{2}-\tau}\left(\sqrt{t_{2}-\tau}+\sqrt{t_{1}-\tau}\right)} } \\
&<\sqrt{t_{2}-t_{1}} \int_{t}^{t_{1}} \frac{d \tau}{\sqrt{t t_{1}-\tau}\left(\sqrt{t_{2}-t_{1}}+\sqrt{t_{1}-\tau}\right)} \\
& \quad=2 \sqrt{t_{2}-t_{1}} \log \frac{\sqrt{t_{2}-t_{1}}+\sqrt{t_{1}-\bar{t}}}{\sqrt{t_{2}-t_{1}}}<K \sqrt{t_{2}-t_{1}}\left|\log \left(t_{2}-t_{1}\right)\right| .
\end{aligned}
$$

Therefore

$$
\text { (3.96) } \begin{aligned}
\int_{\dot{t}}^{t_{1}} & {\left[\frac{1}{\sqrt{t_{1}-\tau}}-\frac{1}{\sqrt{t_{2}-\tau}}\right]\left[\int_{0}^{1}\left|\dot{y}\left(\tau+\theta\left(t_{2}-\tau\right)\right)-\dot{y}\left(\tau+\theta\left(t_{1}-\tau\right)\right)\right| d \theta\right] d \tau } \\
& <K \sqrt{t_{2}-t_{1}}\left|\log \left(t_{2}-t_{1}\right)\right|\left[\frac{\sqrt{t_{2}-t_{1}}}{\bar{t}}+|\log \bar{t}|^{1 / 2}\left(t_{2}-t_{1}\right)^{3 / 4}+\frac{t_{2}-t_{1}}{\bar{t}^{3}}\right] .
\end{aligned}
$$

By applying (3.95) and (3.96) on (3.94), we obtain

$$
\begin{aligned}
& \left|\int_{0}^{t_{1}}\left[\psi\left(t_{2}, \tau\right)-\psi\left(t_{1}, \tau\right)\right]\left[\frac{1}{\sqrt{t_{1}-\tau}}-\frac{1}{\sqrt{t_{2}-\tau}}\right] d \tau\right| \\
& <K\left\{( t _ { 2 } - t _ { 1 } ) | \operatorname { l o g } ( t _ { 2 } - t _ { 1 } ) | \left[\frac{1}{\bar{t}}+|\log \bar{t}|^{1 / 2}\left(t_{2}-t_{1}\right)^{1 / 4}\right.\right. \\
& \left.\left.\quad+\frac{\sqrt{t_{2}-t_{1}}}{\bar{t}^{3}}\right]+\left(t_{2}-t_{1}\right)^{3 / 4}\right\}
\end{aligned}
$$

for $2 \bar{t}<t_{1}<t_{2}$.

We next consider the fourth term on the right hand side of (3. 90) :

$$
\begin{aligned}
& \left|\psi\left(t_{2}, t_{2}\right)\left[\int_{0}^{t_{2}} \frac{d \tau}{\sqrt{t_{2}-\tau}}-\int_{0}^{t_{1}} \frac{d \tau}{\sqrt{t_{1}-\tau}}\right]\right|<K \sqrt{t_{2}}-\sqrt{t_{1}} \\
& \quad<\frac{K\left(t_{2}-t_{1}\right)}{\sqrt{\bar{t}}} \quad\left(\text { for } \bar{t}<t_{1}<t_{2}\right) .
\end{aligned}
$$

Finally, we again use (3.92) for the last term of (3.90):

$$
\begin{aligned}
& \int_{t_{1}}^{t_{2}} \frac{\psi\left(t_{2}, \tau\right)-\psi\left(t_{2}, t_{2}\right)}{\sqrt{t_{2}-\tau}} d \tau \mid<K\left[\int _ { t _ { 1 } } ^ { t _ { 2 } } \left\{\frac{1}{\bar{t}}+|\log \bar{t}|^{1 / 2}\left(t_{2}-\tau\right)^{1 / 4}\right.\right. \\
& \left.\left.\quad+\frac{1}{\bar{t}^{3}} \sqrt{t_{2}-\tau}\right\} d t\right] \\
& \left.<K\left(t_{2}-t_{1}\right)\left[\frac{1}{\bar{t}}+|\log \bar{t}|^{1 / 2}\left(t_{2}-t_{1}\right)^{1 / 4}+\frac{1}{\bar{t}^{3}} \sqrt{t_{2}-t_{1}}\right] \text { (for } \bar{t}<t_{1}<t_{2}\right) .
\end{aligned}
$$

In conclusion, by applying (3.91), (3.68), (3.93), (3.97), (3.98) and (3.99) upon (3.90), we obtain

$$
\text { (3. 100) } \begin{aligned}
\mid & V_{411}\left(t_{2}\right)-V_{411}\left(t_{1}\right) \mid<\mathrm{K}\left[\left|\log \left(t_{2}-t_{1}\right)\right|^{1 / 2}\left(t_{2}-t_{1}\right)^{3 / 4}\right. \\
& \left.+|\log \bar{t}|^{1 / 2}\left(t_{2}-t_{1}\right)^{3 / 4}+\frac{1}{\bar{t}}\left(t_{2}-t_{1}\right)\left|\log \left(t_{2}-t_{1}\right)\right|+\frac{1}{\bar{t}^{3}}\left(t_{2}-t_{1}\right)\right]
\end{aligned}
$$


for $t_{2}>t_{1}>2 \bar{t}$.

For $V_{412}(t)$, we can transfer the estimation for $\bar{V}(t)$ in $\left.\mathrm{ii}^{\prime}\right)$, as before. So, we have

(3. 101) $\left|V_{412}\left(t_{2}\right)-V_{412}\left(t_{1}\right)\right|<K\left[\frac{1}{\bar{t}^{3}}\left(t_{2}-t_{1}\right)+\frac{1}{\bar{t}}\left(t_{2}-t_{1}\right)\left|\log \left(t_{2}-t_{1}\right)\right|\right]$

$$
\left(t_{2}>t_{1}>2 \bar{t}\right) \text {. }
$$

By combining (3.100) and (3.101) we obtain

(3. 102) $\left|V_{41}\left(t_{2}\right)-V_{41}\left(t_{1}\right)\right|<K\left[\left|\log \left(t_{2}-t_{1}\right)\right|^{1 / 2}\left(t_{2}-t_{1}\right)^{3 / 4}\right.$

$$
\left.+|\log \bar{t}|^{1 / 2}\left(t_{2}-t_{1}\right)^{3 / 4}+\frac{1}{\bar{t}}\left(t_{2}-t_{1}\right)\left|\log \left(t_{2}-t_{1}\right)\right|+\frac{1}{\bar{t}^{3}}\left(t_{2}-t_{1}\right)\right] \text {, }
$$

for $t_{2}>t_{1}>2 \bar{t}$.

Similarly, we also have

(3. 103) $\left|V_{42}\left(t_{2}\right)-V_{42}\left(t_{1}\right)\right|<K\left[\left|\log \left(t_{2}-t_{1}\right)\right|^{1 / 2}\left(t_{2}-t_{1}\right)^{3 / 4}\right.$

$$
\left.+|\log \bar{t}|^{1 / 2}\left(t_{2}-t_{1}\right)^{3 / 4}+\frac{1}{\bar{t}}\left(t_{2}-t_{1}\right)\left|\log \left(t_{2}-t_{1}\right)\right|+\frac{1}{\bar{t}^{3}}\left(t_{2}-t_{1}\right)\right] \text {, }
$$

for $t_{2}>t_{1}>2 \bar{t}$.

3.6. 12. vii') For $V_{51}$ and $V_{52}$, we again repeat the discussion for $V_{412}$, as before. So, we have

(3. 104) $\left|V_{51}\left(t_{2}\right)-V_{51}\left(t_{1}\right)\right|<K\left[\frac{1}{\bar{t}^{3}}\left(t_{2}-t_{1}\right)+\frac{1}{\bar{t}}\left(t_{2}-t_{1}\right)\left|\log \left(t_{2}-t_{1}\right)\right|\right]$

$$
\left|V_{52}\left(t_{2}\right)-V_{52}\left(t_{1}\right)\right|<K\left[\frac{1}{\bar{t}}\left(t_{2}-t_{1}\right)\left|\log \left(t_{2}-t_{1}\right)\right|\right] \quad \begin{aligned}
& \left(t_{2}>t_{1}>2 \bar{t}\right), \\
& \left(t_{2}>t_{1}>2 \bar{t}\right) .
\end{aligned}
$$

Consequently, we obtain the revised estimation, from (3.47), $(3.86),(3.87),(3.89),(3.58),(3.59),(3.61),(3.102),(3.103)$, (3.104) and (3.44),

$$
\begin{aligned}
& \left|u\left(y\left(t_{2}\right), t_{2}\right)-u\left(y\left(t_{1}\right), t_{1}\right)\right| \\
& \quad<K\left[\left|\log \left(t_{2}-t_{1}\right)\right|^{1 / 2}\left(t_{2}-t_{1}\right)^{3 / 4}+|\log \bar{t}|^{1 / 2}\left(t_{2}-t_{1}\right)^{3 / 4}\right. \\
& \left.\quad+\frac{1}{\bar{t}}\left(t_{2}-t_{1}\right)\left|\log \left(t_{2}-t_{1}\right)\right|+\frac{1}{\bar{t}^{3}}\left(t_{2}-t_{1}\right)\right] \quad\left(t_{2}>t_{1}>2 \bar{t}\right) .
\end{aligned}
$$

Only by replacing $2 \bar{t}$ by a new $\bar{t}$, we have proved Lemma 3.4.

3. 7. We are now in a position to prove that there exist both

$$
\lim _{x \rightarrow y(t)-0} \frac{\partial u}{\partial x}(x, t)=\frac{\partial u}{\partial x}(y(t)-0, t)
$$


and

$$
\lim _{x \rightarrow y(t)+0} \frac{\partial u}{\partial x}(x, t)=\frac{\partial u}{\partial x}(y(t)+0, t)
$$

for every $t>0$, and they are continuous in $t(t>0)$.

3. 7.1. For the purpose, we will give an expression of the solution $u(x, t)$ using the so-called double layer potential. Put

$$
\text { (3. 107) } \begin{aligned}
u(x, t) & =\int_{0}^{y(\bar{t})} U_{1}(x-\xi, t-\bar{t}) u(\xi, \bar{t}) d \xi+\int_{\bar{t}}^{t} \frac{\partial U_{1}}{\partial \xi}(x, t-\tau) v(\tau) d \tau \\
+ & \int_{\bar{t}}^{t} \frac{\partial U_{1}}{\partial \xi}(x-y(\tau), t-\tau) w(\tau) d \tau
\end{aligned}
$$

in the region $\{0<x<y(t), t>\bar{t}\}$ with any fixed $\bar{t}$, where $v(t)$ and $w(t)$ are unknown functions. We can introduce a system of integral equations to find $v(t)$ and $w(t)$ as follows: we first note relations similar to (3.41) and (3.42), i. e.

$$
\lim _{x \rightarrow 0} \int_{t}^{t} \frac{\partial U_{1}}{\partial \xi}(x, t-\tau) v(\tau) d \tau=\frac{c_{1}}{2 a_{1}} v(t)+\int_{t}^{t} \frac{\partial U_{1}}{\partial \xi}(0, t-\tau) v(\tau) d \tau=\frac{c_{1}}{2 a_{1}} v(t)
$$

(since the last integral term vanishes) and

$$
\text { (3. 108) } \begin{gathered}
\lim _{x \rightarrow y(t)-0} \int_{\bar{t}}^{t} \frac{\partial U_{1}}{\partial \xi}(x-y(\tau), t-\tau) w(\tau) d \tau=-\frac{c_{1}}{2 a_{1}} w(t) \\
+\int_{\bar{t}}^{t} \frac{\partial U_{1}}{\partial \xi}(y(t)-y(\tau), t-\tau) w(\tau) d \tau .
\end{gathered}
$$

From (3. 107), we then have by taking $x \rightarrow 0$ and $x \rightarrow-0(y t)-0$

(3. 109) $\frac{c_{1}}{2 a_{1}} v(t)+\int_{\bar{t}}^{t} \frac{\partial U_{1}}{\partial \xi}(-y(\tau), t-\tau) w(\tau) d \tau$

$$
\begin{aligned}
&= f_{1}(t)-\int_{0}^{y(\bar{t})} U_{1}(-\xi, t-\bar{t}) u(\xi, \bar{t}) d \xi \\
&-\frac{c_{1}}{2 a_{1}} w(t)+\int_{\bar{t}}^{t} \frac{\partial U_{1}}{\partial \xi}(y(t), t-\tau) v(\tau) d \tau \\
& \quad+\int_{\bar{t}}^{t} \frac{\partial U_{1}}{\partial \xi}(y(t)-y(\tau), t-\tau) w(\tau) d \tau \\
&=u(y(t), t)-\int_{0}^{y(\bar{t})} U_{1}(y(t)-\xi, t-\bar{t}) u(\xi, \bar{t}) d \xi .
\end{aligned}
$$

This is a system of integral equations of the Volterra type with kernels of the type

$$
\frac{Q(t, \tau)}{\sqrt{t-\tau}}(Q(t, \tau) \text { is bounded and continuous), }
$$


and with continuous right hand side. As well known, it then have a unique continuous solution $(v, w)$. Further, we can derive estimations for the difference $v\left(t_{2}\right)-v\left(t_{1}\right)$ and $w\left(t_{2}\right)-w\left(t_{1}\right)$ for $t_{2}>t_{1}>3 \bar{t}$ from (3.109). In fact, the integral

$$
\begin{aligned}
& \int_{\bar{t}}^{t} \frac{\partial U_{1}}{\partial \xi}(-y(\tau), t-\tau) w(\tau) d \tau, \int_{\bar{t}}^{t} \frac{\partial U_{1}}{\partial \xi}(y(t), t-\tau) v(\tau) d \tau, \\
& \int_{0}^{y(t)} U_{1}(-\xi, t-\bar{t}) u(\xi, \bar{t}) d \xi \text { and } \int_{0}^{y(\bar{t})} U_{1}(y(t)-\xi, t-\bar{t}) u(\xi, \bar{t}) d \xi
\end{aligned}
$$

have uniformly bounded derivatives with respect to time $t$ for $t>2 \bar{t}$, $f_{1}(t)$ is Lipshitz continuous, and $u(y(t), t)$ satisfies (3.46) (of Lemma 3.4). In addition, the integral

$$
\int_{\bar{t}}^{t} \frac{\partial U_{1}}{\partial \xi}(y(t)-y(\tau), t-\tau) w(\tau) d \tau
$$

goes along the same line of discussion as for $V_{411}(t)$ in 3.6. Therefore, we first get

$$
\left|w\left(t_{2}\right)-w\left(t_{1}\right)\right|<K\left[\frac{\sqrt{t_{2}-t_{1}}}{\bar{t}}+|\log \bar{t}|^{1 / 2}\left(t_{2}-t_{1}\right)^{3 / 4}+\frac{t_{2}-t_{1}}{\bar{t}^{3}}\right]
$$

$$
\left(t_{2}>t_{1}>2 \bar{t}\right)
$$

(see $(3.46)$ and $(3.69)$ ) and

$$
\left|v\left(t_{2}\right)-v\left(t_{1}\right)\right|<K\left(t_{2}-t_{1}\right) \quad\left(t_{2}>t_{1}>2 \bar{t}\right) .
$$

Again by using the obtained (3.110), we get a revised estimation:

(3.112) $\left|w\left(t_{2}\right)-w\left(t_{1}\right)\right|<K\left[\left|\log \left(t_{2}-t_{1}\right)\right|^{1 / 2}\left(t_{2}-t_{1}\right)^{3 / 4}+|\log \bar{t}|^{1 / 2}\left(t_{2}-t_{1}\right)^{3 / 4}\right.$

$$
\left.+\frac{1}{\bar{t}}\left(t_{2}-t_{1}\right)\left|\log \left(t_{2}-t_{1}\right)\right|+\frac{1}{\bar{t}^{3}}\left(t_{2}-t_{1}\right)\right] \quad\left(t_{2}>t_{1}>3 \bar{t}\right) .
$$

3.7.2. Now, we consider $\frac{\partial u}{\partial x}$ from (3.107):

$$
\begin{aligned}
& \frac{\partial u}{\partial x}(x, t)=\int_{0}^{y(\bar{t})} \frac{\partial U_{1}}{\partial x}(x-\xi, t-\bar{t}) u(\xi, \bar{t}) d \xi \\
& +\int_{\bar{t}}^{t} \frac{\partial^{2} U_{1}}{\partial x \partial \xi}(x, t-\tau) v(\tau) d \tau+\int_{\bar{t}}^{t} \frac{\partial^{2} U_{1}}{\partial x \partial \xi}(x-y(\tau), t-\tau) w(\tau) d \tau
\end{aligned}
$$

Clearly, the first and second term on the right hand side of (3.113) are continuous at $(y(t), t)$. To be discussed is the third term. Put 


$$
\begin{aligned}
& \text { (3.114) } \begin{aligned}
\int_{\bar{t}}^{t} & \frac{\partial^{2} U_{1}}{\partial x \partial \xi}(x-y(\tau), t-\tau) w(\tau) d \tau \\
= & -\int_{\bar{t}}^{t} \frac{\partial^{2} U_{1}}{\partial x \partial \xi}(x-y(\tau), t-\tau)[w(t)-w(\tau)] d \tau \\
& +w(t) \int_{\bar{t}}^{t} \frac{\partial^{2} U_{1}}{\partial x \partial \xi}(x-y(\tau), t-\tau) d \tau .
\end{aligned}
\end{aligned}
$$

Here,

$$
\text { (3. 115) } \begin{aligned}
\frac{\partial^{2} U_{1}}{\partial x \partial \xi}(x-\xi, t-\tau)=\frac{c_{1}}{a_{1}} \frac{\partial U_{1}}{\partial \tau}(x-\xi, t-\tau) \\
\quad=\frac{1}{4 \sqrt{\pi}} \frac{\left(\frac{c_{1}}{a_{1}}\right)^{3 / 2}}{(t-\tau)^{3 / 2}}\left[1-\frac{c_{1}(x-\xi)^{2}}{2 a_{1}(t-\tau)}\right] \exp \left(-\frac{c_{1}(x-\xi)^{2}}{4 a_{1}(t-\tau)}\right)
\end{aligned}
$$

So, it is necessary for the first integral on the right hand side of (3.114) to consider

$$
H_{1}=\int_{t}^{t} \frac{w(t)-w(\tau)}{(t-\tau)^{3 / 2}} \exp \left(-\frac{c_{1}(x-y(\tau))^{2}}{4 a_{1}(t-\tau)}\right) d \tau
$$

and

$$
H_{2}=\int_{\bar{t}}^{t} \frac{(w(t)-w(\tau))(x-y(\tau))^{2}}{(t-\tau)^{5 / 2}} \exp \left(-\frac{c_{1}(x-y(\tau))^{2}}{4 a_{1}(t-\tau)}\right) d \tau .
$$

Divide the integral interval into two parts: $(\bar{t}, 3 \bar{t})$ and $(3 \bar{t}, t)$. Then,

$$
\left|H_{1}\right|<K\left[\int_{\bar{t}}^{3 \bar{t}} \frac{d \tau}{(t-\tau)^{3 / 2}}+\int_{3 \bar{t}}^{t} \frac{|w(t)-w(\tau)|}{(t-\tau)^{3 / 2}} d \tau\right]
$$

Applying (3.112) on the second integral, we find that $H_{1}$ converges uniformly in $x$. For $H_{2}$, we first notice that

$$
(x-y(\tau))^{2}<2(x-y(t))^{2}+2(y(t)-y(\tau))^{2}
$$

and

$$
\begin{aligned}
& \exp \left(-\frac{c_{1}(x-y(\tau))^{2}}{4 a_{1}(t-\tau)}\right)=\exp \left(-\frac{c_{1}(x-y(t))^{2}}{4 a_{1}(t-\tau)}\right) \\
& \quad-\exp \left(-\frac{c_{1}(x-y(t))^{2}}{4 a_{1}(t-\tau)}\right)\left\{1-\exp \left(-\frac{c_{1}}{4 a_{1}(t-\tau)}\left[(x-y(\tau))^{2}\right.\right.\right. \\
& \left.\left.\left.-(x-y(t))^{2}\right]\right)\right\}<\exp \left(-\frac{c_{1}(x-y(t))^{2}}{4 a_{1}(t-\tau)}\right)[1+K(|x-y(t)| \\
& \quad+|x-y(\tau)|)]<K \exp \left(-\frac{c_{1}(x-y(t))^{2}}{4 a_{1}(t-\tau)}\right) .
\end{aligned}
$$

Therefore, 


$$
\begin{array}{r}
\left|H_{2}\right|<K\left\{\int_{\bar{t}}^{3 \bar{t}} \frac{d \tau}{(t-\tau)^{5 / 2}}+\int_{3 \bar{t}}^{t} \frac{|w(t)-w(\tau)|(y(t)-y(\tau))^{2}}{(t-\tau)^{5 / 2}} d \tau\right. \\
\left.\quad+\int_{3 \bar{t}}^{t} \frac{|w(t)-w(\tau)|(x-y(t))^{2}}{(t-\tau)^{5 / 2}} \exp \left(-\frac{c_{1}(x-y(t))^{2}}{4 a_{1}(t-\tau)}\right) d \tau\right\} .
\end{array}
$$

The first integral on the right hand side takes a finite value depending only upon $\bar{t}$. Clearly, the second integral converges uniformly in $x$, since $|y(t)-y(\tau)|<K(t-\tau)$. In order to see that the last integral also converges uniformly in $x$, we put

$$
\exp \left(-\frac{c_{1}(x-y(t))^{2}}{4 a_{1}(t-\tau)}\right)=\sigma 。
$$

It is then bounded from above by

$$
\frac{4 a_{1}}{c_{1}} \sup _{t>\tau>3 \bar{t}} \frac{|w(t)-w(\tau)|}{\sqrt{t-\tau}} \int_{0}^{\infty} \exp (-\sigma) d \sigma,
$$

which turns out to be uniformly bounded from (3.112) and the fact that the last integral is equal to 1 . Thus, we have proved that $H_{2}$ also converges uniformly in $x$. Consequently, we have

$$
\begin{gathered}
\lim _{x \rightarrow y(t)-0} \int_{\bar{t}}^{t} \frac{\partial^{2} U_{1}}{\partial x \partial \xi}(x-y(\tau), t-\tau)(w(t)-w(\tau)) d \tau \\
=\int_{t}^{t} \frac{\partial^{2} U_{1}}{\partial x \partial \xi}(y(t)-y(\tau), t-\tau)(w(t)-w(\tau)) d \tau
\end{gathered}
$$

and the limit function is continuous in $t$.

Next, we consider the second integral on the right hand side of (3. 114). Applying (3.115), we have

$$
\begin{aligned}
& \int_{\bar{t}}^{t} \frac{\partial^{2} U_{1}}{\partial x \partial \xi}(x-y(\tau), t-\tau) d \tau=\frac{c_{1}}{a_{1}} \int_{\bar{t}}^{t} \frac{\partial U_{1}}{\partial \tau}(x-y(\tau), t-\tau) d \tau \\
& \quad=\int_{\bar{t}}^{t} \frac{d U_{1}}{d \tau}(x-y(\tau), t-\tau) d \tau-\int_{\bar{t}}^{t} \frac{\partial U_{1}}{\partial \xi}(x-y(\tau), t-\tau) \dot{y}(\tau) d \tau \\
& \quad=-U_{1}(x-y(\bar{t}), t-\bar{t})-\int_{\bar{t}}^{t} \frac{\partial U_{1}}{\partial \xi}(x-y(\tau), t-\tau) \dot{y}(\tau) d \tau
\end{aligned}
$$

The double layer potential here appears. Taking $x \rightarrow y(t)-0$, we get

(3. 117) $\lim _{x \rightarrow y(t)-0} \int_{\bar{t}}^{t} \frac{\partial^{2} U_{1}}{\partial x \partial \xi}(x-y(\tau), t-\tau) d \tau$

$$
\begin{aligned}
=-U_{1}(y(t)-y(\bar{t}), t-\bar{t})+\frac{c_{1}}{2 a_{1}} \dot{y}(t) \\
\quad-\int_{\bar{t}}^{t} \frac{\partial U_{1}}{\partial \xi}(y(t)-y(\tau), t-\tau) \dot{y}(\tau) d \tau
\end{aligned}
$$


(see (3.108)), and the last expression is continuous in $t$. Further, we have found that (3.114) also has a limit function as $x \rightarrow y(t)-0$, and hence that (3.113) and (3.105) has a continuous limit.

The existence of the limit (3.106) and its continuity also are found by the same way.

Thus, we have completed the proof that the constructed solution $(y(t), u(x, t))$ is certainly a local solution of the problem (1.7)(1.13) in the classical sense.

\section{§4. Existence of a Global Solution}

4. 1. The time interval $\left(0, T_{1}\right)$, in which we found a solution, was given so that the constructed solution of difference scheme satisfies the condition (2.29) and the energy inequality (2.28). In the present section, we will show that a solution of difference scheme is always found and hence that a solution of the original problem exists in global in the sense that it does as far as $y(t)$ does not touch the right boundary, $y(t)<1$.

We will start from the fact that, for $t_{n}<T_{1}$

$$
u_{j}^{n}<(1-\mu) u_{E} \quad(0 \leqq j \leqq M)
$$

and

$$
\sum_{j=0}^{M-1} h\left(u_{j x}^{n}\right)^{2}<K
$$

hold. Suppose that $t_{N}$ denotes the maximum discrete time among such $t_{n}$ 's for any fixed $h: t_{N} \leqq T_{1}<t_{N}+k_{N+1}$.

Repeat now the estimation of the initial part in $\S 2$ for $t<t_{N}$, taking $t_{N}$ as a starting point. Just in the same way, we can arrive at the equality of the type (2.36). Adding the estimations of the type (2.37)-(2.39), (2.45)-(2.46) and (2.15), we have

$$
\begin{gathered}
\frac{1}{2} \sum_{n=N+1}^{N_{1}} k_{n}\left(c_{1} \sum_{j=1}^{J_{n}-1}+c_{2} \sum_{j=J_{n}+1}^{M-1}\right) h\left(u_{j \ddagger}^{n}\right)^{2}+\frac{1}{2}\left(a_{1} \sum_{j=0}^{J_{N_{1}}-1}+a_{2} \sum_{j=J_{N_{1}}}^{M-1}\right) h\left(u_{j x}^{N_{1}}\right)^{2} \\
<\frac{\varepsilon_{1}}{2} \sum_{n=N+1}^{N_{1}} k_{n}\left(a_{1} \sum_{j=1}^{J_{n}-1}+a_{2} \sum_{j=J_{n}}^{M-1}\right) h\left(u_{j x}^{n}\right)^{2} \\
\quad+\frac{1}{2}\left[\varepsilon_{2}+\frac{\alpha u_{E}\left(a_{1}-a_{2}\right)}{b}\right] \sum_{n=N+1}^{N_{1}-1} k_{n}\left(u_{J_{n}}^{n}\right)^{2}+
\end{gathered}
$$




$$
\begin{aligned}
& \left.+\frac{1}{2}\left(a_{1} \sum_{j=0}^{J_{N}}+a_{2} \sum_{j=J_{N}+1}^{M-1}\right) h\left(u_{j x}^{N}\right)^{2}\right)+\frac{1}{2}\left(c_{1}+c_{2}\right)|\gamma|^{2}\left(t_{N_{1}}-t_{N}\right) \\
& +\frac{1}{2 \varepsilon_{1}}\left(a_{1}+a_{2}\right)\left|\gamma_{x}\right|^{2}\left(t_{N_{1}}-t_{N}\right)+\frac{\left(\alpha u_{E}\right)^{4}}{2 \varepsilon_{2} b^{2}}\left(t_{N_{1}-1}-t_{N}\right)+\frac{5}{2} \alpha u_{E^{\circ}}^{2}
\end{aligned}
$$

The present problem is to estimate

$$
\sum_{n=N+1}^{N_{1}-1} k_{n}\left(u_{J_{n}}^{n}\right)^{2}
$$

By (2.20), we have $y_{N}>\frac{\alpha}{b} \mu u_{E} T_{1}$. For some positive constant $\delta<$ $\frac{\alpha}{2 b} \mu u_{E} T_{1}$ and sufficiently small $h$, we can then take a value $\bar{x}$ such that

$$
y_{N}-\delta<\bar{x}=\bar{J} h<y_{N}-\frac{\delta}{2} .
$$

Suppose again that, for $N<n \leqq N_{1}-1$,

$$
u_{J_{n}}^{n}<(1-\mu) u_{E}
$$

with some positive constant $\mu<1$. Due to (4.5), (2.30) and Lemma A. 6 , we have a positive constant $A(\delta)$, depending on $\delta$, such that

$$
\left|u_{\bar{J} x}^{n}\right| \text { and }\left|u_{\bar{J} \bar{t}}^{n}\right|<A(\delta) \quad \text { for } N<n \leqq N_{1}-1 \text {. }
$$

Expand $u_{J_{n}^{\bar{x}}}^{n}$ into

$$
u_{J_{n} \bar{x}}^{n}=\frac{c_{1}}{a_{1}} \sum_{j=\bar{J}+1}^{J_{n}-1} h u_{j \bar{t}}^{n}+u_{\bar{J} x}^{n}
$$

instead of (2.41). Hence

$$
\left(u_{J_{n} \tilde{x}}^{n}\right)^{2}<2\left[\left(\frac{c_{1}}{a_{1}}\right)^{2}\left(J_{N_{1}}-J_{N}\right) h \sum_{j=\bar{J}+1}^{J_{n}-1} h\left(u_{j \bar{t}}^{n}\right)^{2}+\left(u_{\bar{J} x}^{n}\right)^{2}\right] \quad\left(n<N_{1}\right)
$$

and, by (2.9) and (2.16),

$$
\left(u_{J_{n} x}^{n}\right)^{2}<4\left[\left(\frac{c_{1}}{a_{2}}\right)^{2}\left(\delta+\frac{\alpha u_{E}}{b}\left(t_{N_{1}}-t_{N}\right) \sum_{j=\bar{J}+1}^{J_{n}-1} h\left(u_{j \bar{t}}^{n}\right)^{2}+\left(\frac{a_{1}}{a_{2}}\right)^{2}\left(u_{\bar{J} J}^{n}\right)^{2}\right]+2\left(\frac{\alpha u_{E}}{a_{2}}\right)^{2} .\right.
$$

Therefore, we get

$$
\begin{aligned}
\sum_{n=N+1}^{N_{1}-1} k_{n}\left(u_{J_{n} x}^{n}\right)^{2} & <4\left(\frac{c_{1}}{a_{2}}\right)^{2}\left[\delta+\frac{\alpha u_{E}}{b}\left(t_{N_{1}}-t_{N}\right)\right] \sum_{n=N+1}^{N_{1}-1} k_{n} \sum_{j=\bar{J}+1}^{J_{n}-1} h\left(u_{j \bar{t}}^{n}\right)^{2} \\
& +4\left(t_{N_{1}}-t_{N}\right)\left[\left(\frac{a_{1}}{a_{2}}\right)^{2} A(\delta)^{2}+\frac{1}{2}\left(\frac{\alpha u_{E}}{a_{2}}\right)^{2}\right] .
\end{aligned}
$$

Applying (4.7) on (4.3), we have 


$$
\begin{gathered}
\sum_{n=N+1}^{N_{1}} k_{n}\left(d_{1}^{\prime} \sum_{j=1}^{J_{n}-1}+c_{2} \sum_{j=J_{n}+1}^{M-1}\right) h\left(u_{j t}^{n}\right)^{2}+\left(a_{1} \sum_{j=0}^{J_{N_{1}}-1}+a_{2} \sum_{j=J_{N_{1}}}^{M-1}\right) h\left(u_{j x}^{N_{1}}\right)^{2} \\
\quad<\varepsilon_{1} \sum_{n=N+1}^{N_{1}} k_{n}\left(a_{1} \sum_{j=1}^{J_{n}-1}+a_{2} \sum_{j=J_{n}}^{M-1}\right) h\left(u_{j x}^{n}\right)^{2}+K
\end{gathered}
$$

where

$$
d_{1}^{\prime}=c_{1}\left\{1-\frac{4 c_{1}}{a_{2}^{2}}\left[\delta+\frac{\alpha u_{E}}{b}\left(t_{N_{1}}-t_{N}\right)\right]\left[\varepsilon_{2}+\frac{\alpha u_{E}}{b}\left(a_{1}-a_{2}\right)\right]\right\}
$$

and

$$
\begin{aligned}
K & =\left(a_{1} \sum_{j=0}^{J_{N}}+a_{2} \sum_{j=J_{N}+1}^{M-1}\right) h\left(u_{j x}^{N}\right)^{2}+5 \alpha u_{E}^{2} \\
& +\left(t_{N_{1}}-t_{N}\right)\left\{4\left[\left(\frac{a_{1}}{a_{2}}\right)^{2} A(\delta)^{2}+\frac{1}{2}\left(\frac{\alpha u_{E}}{a_{2}}\right)^{2}\right]\left[\varepsilon_{2}+\frac{\alpha u_{E}\left(a_{1}-a_{2}\right)}{b}\right]\right. \\
& \left.+\left(c_{1}+c_{2}\right)|\gamma|^{2}+\frac{1}{\varepsilon_{1}}\left(a_{1}+a_{2}\right)\left|\gamma_{x}\right|^{2}+\frac{\left(\alpha u_{E}\right)^{4}}{\varepsilon_{2} b^{2}}\right\} .
\end{aligned}
$$

We will fix $\varepsilon_{2}, \delta$ and $\varepsilon_{1}$ as follows: put

$$
\max \left\{1, \frac{\alpha u_{E}}{b}\left(a_{1}-a_{2}\right)\right\}=\kappa,
$$

and take $\varepsilon_{2}$ so small that $\varepsilon_{2}<\kappa$. Further, take $\delta$ so small that

$$
\delta<\min \left\{\frac{a_{2}^{2} \kappa}{16 c_{1}}, \frac{\alpha}{2 b} \mu u_{E} \Delta T\right\}
$$

where

$$
\Delta T=\frac{a_{2}^{2} b}{16 c_{1} \alpha u_{E}} .
$$

We then have $d_{1}^{\prime}>\frac{c_{1}}{2}$, if

$$
t_{N_{1}}-t_{N}<\Delta T \text {. }
$$

Then, it follows from (4.8) that

$$
\begin{aligned}
& \sum_{n=N+1}^{N_{1}} k_{n}\left(\frac{c_{1}}{2} \sum_{j=1}^{J_{n}-1}+c_{2} \sum_{j=J_{n}+1}^{M-1}\right) h\left(u_{j i}^{n}\right)^{2}+\left(a_{1} \sum_{j=0}^{J_{N_{1}}-1}+a_{2} \sum_{j=J_{N_{1}}}^{M-1}\right) h\left(u_{j x}^{N_{1}}\right)^{2} \\
& \quad<\varepsilon_{1} \sum_{n=N+1}^{N_{1}} k_{n}\left(a_{1} \sum_{j=1}^{J_{n}-1}+a_{2} \sum_{j=J_{n}}^{M-1}\right) h\left(u_{j x}^{n}\right)^{2}+K .
\end{aligned}
$$

Take the procedure used to get (2.53) from (2.51). Then, by putting $\varepsilon_{1}=\frac{1}{2 \Delta T}$, we obtain 


$$
\sum_{n=N+1}^{N_{1}} k_{n}\left(\frac{c_{1}}{2} \sum_{j=1}^{J_{n}-1}+c_{2} \sum_{j=J_{n}+1}^{M-1}\right) h\left(u_{j i}^{n}\right)^{2}+\left(a_{1} \sum_{j=0}^{J_{N_{1}}-1}+a_{2} \sum_{j=J_{N_{1}}}^{M-1}\right) h\left(u_{j x}^{N_{1}}\right)^{2}<K
$$

for $T_{1}<t_{N_{1}}<T_{2}^{\prime}=T_{1}+\Delta T$, under the assumption (4.5). If this assumption were to be satisfied for $T_{1} \leqq t_{N_{1}-1}<T_{2}^{\prime}$ for all $h<h_{0}$, we could again construct a solution of the original problem for $T_{1} \leqq t<T_{2}^{\prime}$, too. Further, if the assumption were to hold for some interval $\left(T_{2}^{\prime}, T_{1}+p \Delta T\right)$ ( $p$ :integer) for all $h<h_{0}$, we could repeat the procedure to get a solution for $\left(T_{1}+(q-1) \Delta T, T_{1}+q \Delta T\right), q=2,3, \ldots, p$, successively. Suppose, in addition to such situation, that we see a time $t_{\bar{n}}$ in $\left(T_{1}+p \Delta T, T_{1}+(p+1) \Delta T\right)$, at which (4.5) is first violated with some $h\left(<h_{0}\right)$, Consider the lower limit of such $t_{\bar{n}}$ as $h \rightarrow 0$ :

$$
\lim _{\overline{h \rightarrow 0}} t_{\bar{n}}=T_{2}^{\prime \prime}=T_{0}+(p+\eta) \Delta T \quad(0 \leqq \eta<1) 。
$$

It then follows that we can take an $h_{1}\left(<h_{0}\right)$ such that $(4.5)$ is satisfied for all $t_{n}<T_{1}+\left(p+\frac{\eta}{2}\right) \Delta T$ and all $h<h_{1}$. So we have a solution for the time interval $\left(T_{1}+p \Delta T, T_{1}+\left(p+\frac{\eta}{2}\right) \Delta T\right)$. Thus, we could find the solution for

$$
0<t<T_{2}=T_{1}+\left(p+\frac{\eta}{2}\right) \Delta T
$$

4. 2. The next problem is to continue the solution beyond $T_{2}$. For it, we replace the constant $\mu$ by $\mu / 2$, so as to have a next time interval $\left(T_{2}, T_{3}\right)$, on which the condition $u_{J_{n}}^{n}<\left(1-\frac{\mu}{2}\right) u_{E}$ is assured for all $h<h_{2}, h_{2}$ being another constant $\left(<h_{1}\right)$, and hence to find a solution on the interval. Further, taking a sequence $\mu / 2^{i}, i=2,3,4$, $\ldots$, we have a sequence of constant, $h_{i}, i=2,3,4, \ldots$, and that of time interval $\left(T_{i}, T_{i+1}\right), i=2,3,4, \ldots$, in each of which the condition

$$
u_{J_{n}}^{n}<\left(1-\frac{\mu}{2^{i}}\right) u_{E}
$$

is satisfied for all $h<h_{i}$, and hence a solution can be constructed by the method already mentioned. It is clear that the obtained sequence of solutions constitutes a solution for any time interval $\left(0, T_{n}\right)$, as a whole. It must be here noticed that the internal boundary value $u(y(t), t)$ never attain $u_{E}$ at a finite time. In fact, suppose that it attains at $\bar{T}$. It then follows from Friedman's Lemma (see, for 
example [6]) that

$$
\frac{\partial u}{\partial x}(y(\bar{T})-0, \bar{T})>0 \text { and } \frac{\partial u}{\partial x}(y(\bar{T})+0, \bar{T})<0
$$

(under the assumption of Lemma 2.1.) But, this is a contradiction to the internal boundary condition (1.9).

Thus, we have found a solution of the original problem for all $t>0$.

Existence Theorem. Assume that $\phi(x), f_{1}(t)$ and $f_{2}(t)$ are Lipshitz continuous and satisfy the inequality

$$
0<\phi(x), f_{1}(t) \text { and } f_{2}(t)<u_{E}
$$

for all $t>0$. Then, there exists a classical solution of the problem (1.7)(1.13) while $y(t)$ is far from the right boundary $(x=1)$.

\section{§5. Uniquness of Solution}

In the present section, we will show a uniqueness theorem for the problem (1.7)-(1.13) by adding one more condition

$$
\left(c_{2}-c_{1}\right) u_{E}<b
$$

which means that the given initial degree of supercooling is not so much.

Note first that we have some equalities from (1.7)-(1.11): immediately from (1.9)-(1.10),

$$
\begin{aligned}
y(t) & =\frac{1}{b} \int_{0}^{t}\left[a_{1} \frac{\partial u}{\partial x}(y(\tau)-0, \tau)-a_{2} \frac{\partial u}{\partial x}(y(\tau)+0, \tau)\right] d \tau \\
& =\frac{\alpha}{b} \int_{0}^{t}\left[u_{E}-u(y(\tau), \tau)\right] d \tau
\end{aligned}
$$

and by integrating both (1.7) and (1.8) over the region $\{0<x<y(\tau)$, $0<\tau<t\}$ and $\{y(\tau)<x<1,0<\tau<t\}$, respectively,

$$
\begin{aligned}
& c_{1}\left[\int_{0}^{y(t)} u(x, t) d x-\int_{0}^{t} u(y(\tau), \tau) \dot{y}(\tau) d \tau\right] \\
& =a_{1}\left[\int_{0}^{t} \frac{\partial u}{\partial x}(y(\tau)-0, \tau) d \tau-\int_{0}^{t} \frac{\partial u}{\partial x}(0, \tau) d \tau\right], \\
& c_{2}\left[\int_{y(t)}^{1} u(x, t) d x+\int_{0}^{t} u(y(\tau), \tau) \dot{y}(\tau) d \tau-\int_{0}^{1} \phi(x) d x\right] \\
& \left.=a_{2}\left[\int_{0}^{t} \frac{\partial u}{\partial x}(1, \tau) d \tau-\int_{0}^{t} \frac{\partial u}{\partial x}(y(\tau)+0, \tau) d \tau\right)\right],
\end{aligned}
$$


and further by adding the last two relations and using (1.9),

$$
\begin{aligned}
& c_{1} \int_{0}^{y(t)} u(x, t) d x+c_{2} \int_{y(t)}^{1} u(x, t) d x-c_{2} \int_{0}^{1} \phi(x) d x \\
& \quad=a_{2} \int_{0}^{t} \frac{\partial u}{\partial x}(1, \tau) d \tau-a_{1} \int_{0}^{t} \frac{\partial u}{\partial x}(0, \tau) d \tau+\left[b-\left(c_{2}-c_{1}\right) u_{E}\right] y(t) \\
& \quad+\frac{b}{\alpha}\left(c_{2}-c_{1}\right) \int_{0}^{t} \dot{y}(\tau)^{2} d \tau .
\end{aligned}
$$

Suppose now that we have two solutions, $\left(y_{i}(t), u_{i}(x, t)\right)(i=1,2)$ with same data, which are different each other in a time interval $(0, \varepsilon]$ and satisfy

$$
\dot{y}_{1}(t)<\dot{y}_{2}(t) \quad(0<t \leqq \varepsilon) .
$$

This also means that

$$
y_{1}(t)<y_{2}(t) \quad(0<t \leqq \varepsilon)
$$

(due to $y_{1}(0)=y_{2}(0)=0$ ) and

$$
u_{1}\left(y_{1}(t), t\right)>u_{2}\left(y_{2}(t), t\right) \quad(0<t \leqq \varepsilon)
$$

(due to $(1.9))$.

Let us show that the above assumption leads to contradiction. We first find that the condition (5.4)-(5.6) do not allow such a time interval $\left(0, \varepsilon^{\prime}\right] \quad\left(\varepsilon^{\prime}<\varepsilon\right)$, in which

$$
\begin{gathered}
u_{1}(x, t)>u_{2}(x, t) \text { for } 0<x<y_{1}(t) \text { and } y_{2}(t)<x<1, \\
u_{1}(x, t) \geqq u_{2}(x, t) \text { for } y_{1}(t) \leqq x \leqq y_{2}(t)
\end{gathered}
$$

always hold. In fact, by subtracting the relation (5.3) applied for $\left(y_{2}(t), u_{2}(x, t)\right)$ from that for $\left(y_{1}(t), u_{1}(x, t)\right)$, we get

$$
\begin{gathered}
c_{1} \int_{0}^{y_{1}\left(\varepsilon^{\prime}\right)}\left[u_{1}\left(x, \varepsilon^{\prime}\right)-u_{2}\left(x, \varepsilon^{\prime}\right)\right] d x+\int_{y_{1}\left(\varepsilon^{\prime}\right)}^{y_{2}^{\left(\varepsilon^{\prime}\right)}}\left[c_{2} u_{1}\left(x, \varepsilon^{\prime}\right)-c_{1} u_{2}\left(x, \varepsilon^{\prime}\right)\right] d x \\
+c_{2} \int_{y_{2}\left(\varepsilon^{\prime}\right)}^{1}\left[u_{1}\left(x, \varepsilon^{\prime}\right)-u_{2}\left(x, \varepsilon^{\prime}\right)\right] d x \\
=a_{2} \int_{0}^{\varepsilon^{\prime}}\left[\frac{\partial u_{1}}{\partial x}(1, t)-\frac{\partial u_{2}}{\partial x}(1, t)\right] d t-a_{1} \int_{0}^{\varepsilon^{\prime}}\left[\frac{\partial u_{1}}{\partial x}(0, t)\right. \\
\left.\quad-\frac{\partial u_{2}}{\partial x}(0, t)\right] d t \\
+\left[b-\left(c_{2}-c_{1}\right) u_{E}\right]\left[y_{1}\left(\varepsilon^{\prime}\right)-y_{2}\left(\varepsilon^{\prime}\right)\right] \\
\quad+\frac{b}{\alpha}\left(c_{2}-c_{1}\right) \cdot \int_{0}^{\varepsilon^{\prime}}\left[\dot{y}_{1}^{2}(t)-\dot{y}_{2}^{2}(t)\right] d t .
\end{gathered}
$$


If (5.7) were to hold, the left hand side of (5.8) should be positive due to (1.13), while its right hand side be negative by (5.1), (5.4), (5.6) and the inequalities,

$$
\begin{array}{ll}
\frac{\partial u_{1}}{\partial x}(1, t)-\frac{\partial u_{2}}{\partial x}(1, t) \leqq 0 & \left(0<t \leqq \varepsilon^{\prime}\right), \\
\frac{\partial u_{1}}{\partial x}(0, t)-\frac{\partial u_{2}}{\partial x}(0, t) \geqq 0 & \left(0<t \leqq \varepsilon^{\prime}\right),
\end{array}
$$

which themselves follow from the assumption (5.7).

The next matters which may happen under our assumption (5.4)(5.6) are that, for a sufficiently small time interval $\left(0, \varepsilon^{\prime}\right]$,

$$
u_{1}\left(y_{2}(t), t\right)>u_{2}\left(y_{2}(t), t\right)
$$

always holds. We may have, in fact, more stringent matters that there are not any time interval $\left(0, \varepsilon^{\prime}\right]$ in which, for some function $Z(t), y_{1}(t) \leqq Z(t) \leqq y_{2}(t) \quad\left(0 \leqq t \leqq \varepsilon^{\prime}\right)$,

$$
u_{1}(Z(t), t) \leqq u_{2}\left(y_{2}(t), t\right)
$$

always holds. In fact, suppose that we have such an interval $\left[0, \varepsilon^{\prime}\right]$, and we then find from (5.2) that, for $0<t \leqq \varepsilon^{\prime}$,

$$
\begin{aligned}
0<y_{2}(t)-y_{1}(t) & =-\frac{\alpha}{b} \int_{0}^{t}\left[u_{2}\left(y_{2}(\tau), \tau\right)-u_{1}\left(y_{1}(\tau), \tau\right)\right] d \tau \\
& \leqq-\frac{\alpha}{b} \int_{0}^{t}\left[u_{1}(Z(\tau), \tau)-u_{1}\left(y_{1}(\tau), \tau\right)\right] d \tau .
\end{aligned}
$$

Here

$$
\begin{aligned}
& \int_{0}^{t}\left[u_{1}(Z(\tau), \tau)-u_{1}\left(y_{1}(\tau), \tau\right)\right] d \tau=\int_{0}^{t}\left[\int_{y_{1}(\tau)}^{Z(\tau)} \frac{\partial u_{1}}{\partial x}(x, \tau) d x\right] d \tau \\
= & \int_{0}^{t}\left\{\left[Z(\tau)-y_{1}(\tau)\right] \frac{\partial u_{1}}{\partial x}(Z(\tau), \tau)-\int_{y_{1}(\tau)}^{Z(\tau)}\left[x-y_{1}(\tau)\right] \frac{\partial^{2} u_{1}}{\partial x^{2}}(x, \tau) d x\right\} d \tau
\end{aligned}
$$

and hence

$$
\begin{aligned}
& \left|\int_{0}^{t} u_{1}(Z(\tau), \tau)-u_{1}\left(y_{1}(\tau), \tau\right) d \tau\right| \\
& \quad<\max _{0 \leq \tau \leq t}\left(Z(\tau)-y_{1}(\tau)\right) \int_{0}^{t}\left|\frac{\partial u_{1}}{\partial x}(Z(\tau), \tau)\right| d \tau \\
& \quad+\left\{\int_{0}^{t} \int_{y_{1}(\tau)}^{Z(\tau)}\left(x-y_{1}(\tau)\right)^{2} d x d \tau\right\}^{1 / 2}\left\{\int_{0}^{t} \int_{y_{1}(\tau)}^{Z(\tau)}\left|\frac{\partial^{2} u_{1}}{\partial x^{2}}(x, \tau)\right|^{2} d x d \tau\right\}^{1 / 2} \\
& \quad<\max _{0 \leq \tau \leq t}\left(y_{2}(\tau)-y_{1}(\tau)\right) \sqrt{t}\left(\int_{0}^{t}\left|\frac{\partial u_{1}}{\partial x}(Z(\tau), \tau)\right|^{2} d \tau\right)^{1 / 2} \\
& \quad+\frac{1}{\sqrt{3}} \max _{0 \leqq \tau \leqq t}\left(y_{2}(\tau)-y_{1}(\tau)\right)^{3 / 2} \sqrt{t}\left\{\int_{0}^{t} \int_{y_{1}(\tau)}^{y_{2}(\tau)}\left|\frac{\partial^{2} u_{1}}{\partial x^{2}}(x, \tau)\right|^{2} d x d \tau\right\}^{1 / 2},
\end{aligned}
$$


since both quadratic integrals on the right hand side have finite values for our solution concerned. Now, we put

$$
\max _{0 \leq t \leq \varepsilon^{\prime}}\left(y_{2}(t)-y_{1}(t)\right)=\delta\left(\varepsilon^{\prime}\right) .
$$

Then, we get, from (5.11) and (5.12),

$$
\delta\left(\varepsilon^{\prime}\right)<K \sqrt{\varepsilon^{\prime}}\left(\delta\left(\varepsilon^{\prime}\right)+\delta\left(\varepsilon^{\prime}\right)^{3 / 2}\right) \text {, or } 1<K \sqrt{\varepsilon^{\prime}}\left(1+\sqrt{\delta\left(\varepsilon^{\prime}\right)}\right) .
$$

But the last inequality never holds for sufficiently small $\varepsilon^{\prime}$. This is a contradiction. Therefore, we have

$$
u_{1}(Z(t), t)>u_{2}\left(y_{2}(t), t\right)
$$

and especially (5.10) for a sufficiently small interval, $0<t<\varepsilon^{\prime}$.

On the other hand, it is easily found from the maximum principle that the profiles of $u_{i}(x, t) \quad(i=1,2)$ never meet each other both in $0<x<y_{1}(t)$ and $y_{2}(t)<x<1$ at every time $t, 0<t<\varepsilon_{0}$. Therefore, remained is only the case that such profiles may cross in $y_{1}(t)<x<y_{2}(t)$ for some interval $0<t<\varepsilon^{\prime \prime}$. Then, we can find such smooth function $Y(t)$ that $y_{1}(t)<Y(t)<y_{2}(t) \quad\left(0<t<\varepsilon^{\prime \prime}\right)$ and the followings hold:

$$
\begin{aligned}
& u_{1}(Y(t), t)=u_{2}(Y(t), t) \\
& \frac{\partial u_{1}}{\partial x}(Y(t), t) \geqq \frac{\partial u_{2}}{\partial x}(Y(t), t)
\end{aligned}
$$

and

$$
u_{1}(x, t)>u_{2}(x, t) \text { for } Y(t)<x<y_{2}(t) .
$$

In fact, it follows from the implicit function theorem, since

$$
\frac{\partial^{p} u_{1}}{\partial x^{p}}(Y(t), t)=\frac{\partial^{p} u_{2}}{\partial x^{p}}(Y(t), t) \quad(p=0,1,2, \cdots, i)
$$

and

$$
\frac{\partial^{i+1} u_{1}}{\partial x^{i+1}}(Y(t), t)>\frac{\partial^{i+1} u_{2}}{\partial x^{i+1}}(Y(t), t)
$$

should hold for some $i$. Since $\dot{Y}(0)=\dot{y}_{1}(0)=\dot{y}_{2}(0)=\frac{\alpha u_{E}}{b}>0$, we may consider from continuity of $\dot{Y}(t)$ that

$$
\dot{Y}(t)>0 \quad\left(0<t<\varepsilon^{\prime \prime}\right) \text {. }
$$

By integrating (1.7) and (1.8) in the region $\left\{Y(t)<x<1,0<t<\varepsilon^{\prime \prime}\right\}$ as done to get (5.3), applying the condition (1.9)-(1.10) and practicing integration of $\dot{y}_{2}(t)$, we obtain 
(5. 18)

$$
\begin{aligned}
& \int_{Y\left(\varepsilon^{\prime \prime}\right)}^{y_{2}\left(\varepsilon^{\prime \prime}\right)}\left[c_{2} u_{1}\left(x, \varepsilon^{\prime \prime}\right)-c_{1} u_{2}\left(x, \varepsilon^{\prime \prime}\right)\right] d x+c_{2} \int_{y_{2}\left(\varepsilon^{\prime \prime}\right)}^{1}\left[u_{1}\left(x, \varepsilon^{\prime \prime}\right)-u_{2}\left(x, \varepsilon^{\prime \prime}\right)\right] d x \\
+ & \int_{0}^{\varepsilon^{\prime \prime}}\left[c_{2} u_{1}(Y(t), t)-c_{1} u_{2}(Y(t), t)\right] \dot{Y}(t) d t+\frac{b}{\alpha}\left(c_{2}-c_{1}\right) \int_{0}^{\varepsilon^{\prime \prime}} \dot{y}_{2}(t)^{2} d t \\
= & a_{2} \int_{0}^{\varepsilon^{\prime \prime}}\left[\frac{\partial u_{1}}{\partial x}(1, t)-\frac{\partial u_{2}}{\partial x}(1, t)\right] d t-a_{2} \int_{0}^{\varepsilon^{\prime \prime}} \frac{\partial u_{1}}{\partial x}(Y(t), t) d t \\
+ & a_{1} \int_{0}^{\varepsilon^{\prime \prime}} \frac{\partial u_{2}}{\partial x}(Y(t), t) d t-\left[b-\left(c_{2}-c_{1}\right) u_{E}\right] y_{2}\left(\varepsilon^{\prime \prime}\right) .
\end{aligned}
$$

It follows immediately from (1.13), (5.14), (5.16) and (5.17) that the left hand side of (5.18) is positive. Now, if

$$
\frac{\partial u_{2}}{\partial x}(Y(t), t) \leqq 0 \quad\left(0<t<\varepsilon^{\prime \prime}\right)
$$

were to hold, the right hand side would be negative due to (5.9), (1.13), (5.15) and (5.1). This is a contradiction. Therefore, we should have

$$
\frac{\partial u_{2}}{\partial x}(Y(t), t)>0 \quad\left(0<t<\varepsilon^{\prime \prime}\right) .
$$

On the other hand, by applying (5.13) with $Z(t)=Y(t)$ and (5.14), we have

$$
u_{2}(Y(t), t)>u_{2}\left(y_{2}(t), t\right) \quad\left(0<t<\varepsilon^{\prime \prime}\right) .
$$

But, (5.19) and (5.20) are not compatible with the maximum principle to be satisfied by $u_{2}(x, t)$ in the region $\left\{Y(t)<x<y_{2}(t)\right.$, $\left.0<t<\varepsilon^{\prime \prime}\right\}$.

Thus, we have proved that the assumption (5.4) is not valid, and hence that

$$
y_{1}(t)=y_{2}(t) \text { for some interval, } 0 \leqq t \leqq \varepsilon .
$$

It is then clear that

$$
u_{1}(x, t)=u_{2}(x, t) \text { for } 0<x<1,0<t \leqq \varepsilon_{0}
$$

These mean that the solution of our problem is uniquely determined for $0<t<\varepsilon$, at least.

Clearly, we can repeat the same discussion for a series of time intervals $\left(\varepsilon_{n}, \varepsilon_{n+1}\right) \quad\left(n=1,2 \ldots ; \varepsilon_{1}=\varepsilon\right)$. In conclusion, we arrive at the following theorem:

Uniqueness Theorem. A classical solution of the problem (1.7)-(1.13) 
is uniquely determined under the condition (5.1).

\section{Appendix $\mathbb{A}$}

In this Appendix, it will be shown that the problem of difference scheme

(A. 1)

$$
\left(u_{j}^{n}\right)_{\bar{t}}=\left(u_{j}^{n}\right)_{x \bar{x}} \quad(1 \leqq j \leqq J-1, J h=X=\mathrm{constan} \mathrm{t})
$$

$$
\begin{array}{ll}
u_{j}^{0}=\phi_{j} & (j=1,2, \ldots, J-1) \\
u_{0}^{n}=f^{n}, u_{J}^{n}=g^{n} & (n=1,2,3, \ldots)
\end{array}
$$

with uniformly bounded data $\left\{\phi_{j}\right\},\left\{f^{n}\right\}$ and $\left\{g^{n}\right\}$ has a family of solutions for a sequence of space mesh size $h$ 's, whose difference quotients of any times both in $x$ and $t$ are uniformly bounded in any compact set contained in

$$
\Omega=\{0<x<X, 0<t<T\} .
$$

Here, we have taken the coefficients of heat difference equation to be all one for simplicity. But the following discussion does not give any essential change even for the general case.

To see the above fact, it is essential to have an estimate for $u_{x}$. Such an estimate is well known not only for a heat equation but also for general partial differential equations of parabolic and elliptic type, and it is usally called an estimation of Bernstein type. Similar estimation may be, of course, expected for corresponding difference schemes. In fact, for a pure implicit difference analogue for heat equation with uniform mesh width $h$ and time step $k$, such estimation is known (see [5]). Here, we want to get such estimation for a solution of (A. 1) with variable time steps $\left\{k_{n}\right\}$ 。

\section{A. 1. Green's Function}

For the case of homogeneous boundary condition

$$
f^{n}=g^{n}=0 \quad(n=1,2,3, \ldots),
$$

a solution of (A. 1) is expressed in the form of eigenfunction expansion by using eigenfunctions of a corresponding eigenvalue problem

$$
\text { (A. 3) } \quad \frac{\lambda-1}{k_{n}} u_{j}=\lambda\left(u_{j}\right)_{x \bar{x}} \quad(1 \leqq j \leqq J-1) \text {, and } u_{0}=u_{J}=0 \text {. }
$$

In fact, it is easily seen that its eigenvalues are 
(A. 4) $\lambda=\lambda_{s}=\left(1+4 \kappa_{n} \sin ^{2} \frac{s \pi}{2 J}\right)^{-1}$, where $\kappa_{n}=\frac{k_{n}}{h^{2}}(s=1,2, \ldots, J-1)$ and corresponding eigenfunctions are

$$
u_{j}=u_{j}^{(s)}=\sin \frac{s j \pi}{J}(s=1,2, \ldots, J-1) .
$$

As easily shown, these eigenfunctions are orthogonal one another:

$$
\sum_{j=1}^{J-1} \sin \frac{r j \pi}{J} \sin \frac{s j \pi}{J}=\left\{\begin{array}{l}
0(r \neq s) \\
\frac{J}{2}(r=s)
\end{array} .\right.
$$

It is then sure that any given function $\phi=\left\{\phi_{j}\right\}$ can be expanded as (A. 7)

$$
\phi_{j}=\sum_{s=1}^{J-1} b_{s} \sin \frac{s j \pi}{J}(j=1,2, \ldots, J-1),
$$

where its Fourie coefficients are given by the formula

$$
b_{s}=\frac{2}{J} \sum_{j=1}^{J-1} \phi_{j} \sin \frac{s j \pi}{J}(s=1,2, \ldots, J-1) .
$$

Such expansion of initial data allows us to get an expression of a solution of homogeneous boundary value problem (A.1), (A.2):

$$
\begin{aligned}
u_{r}^{n} & =\sum_{s=1}^{J-1} b_{s} \prod_{q=1}^{n}\left(1+4 \kappa_{q} \sin ^{2} \frac{s \pi}{2 J}\right)^{-1} \sin \frac{r s \pi}{J} \\
& =\frac{2}{J} \sum_{j=1}^{J-1}\left[\sum_{s=1}^{J-1} \prod_{q=1}^{n}\left(1+4 \kappa_{q} \sin ^{2} \frac{s \pi}{2 J}\right)^{-1} \sin \frac{r s \pi}{J} \sin \frac{s j \pi}{J}\right] \phi_{j} .
\end{aligned}
$$

We write it in the form

$$
u_{r}^{n}=\sum_{j=1}^{J-1} h G\left(x_{r}, \xi_{j} ; t_{n}, 0\right) \phi_{j}
$$

where

$$
G\left(x_{r}, \xi_{j} ; t_{n}, 0\right)=\frac{2}{J h} \sum_{s=1}^{J-1} \prod_{q=1}^{n}\left(1+4 \kappa_{q} \sin ^{2} \frac{s \pi}{2 J}\right)^{-1} \sin \frac{r s \pi}{J} \sin \frac{s j \pi}{J} .
$$

In general, we call the function

$$
\text { (A. 10) } G\left(x_{r}, \xi_{j} ; t_{n}, \tau_{p-1}\right)=\frac{2}{J h} \sum_{s=1}^{J-1} \prod_{q=p}^{n}\left(1+4 \kappa_{q} \sin ^{2} \frac{s \pi}{2 J}\right)^{-1} \sin \frac{r s \pi}{J} \sin \frac{s j \pi}{J}
$$

Green's function of the present homogeneous problem. It is verified by direct substitution that the Green's function satisfies the following equations and homogeneous boundary conditions:

$$
\begin{aligned}
& G\left(x_{r}, \xi_{j} ; t_{n}, \tau_{p-1}\right)_{\bar{t}}=G\left(x_{r}, \xi_{j} ; t_{n}, \tau_{p-1}\right)_{x \bar{x}}, \\
& G\left(0, \xi_{j} ; t_{n}, \tau_{p-1}\right)=G\left(x_{J}, \xi_{j} ; t_{n}, \tau_{p-1}\right)=0
\end{aligned}
$$


and

(A. 12)

$$
\begin{aligned}
& G\left(x_{r}, \xi_{j} ; t_{n}, \tau_{p-1}\right)_{\tau}+G\left(x_{r}, \xi_{j} ; t_{n}, \tau_{p-1}\right)_{\xi \xi}=0, \\
& G\left(x_{r}, 0 ; t_{n}, \tau_{p-1}\right)=G\left(x_{r}, \xi_{J} ; t_{n}, \tau_{p-1}\right)=0
\end{aligned}
$$

for $n>p-1$. To put, for $n=p-1$,

$$
\begin{aligned}
& \text { (A.13) } G\left(x_{r}, \xi_{j} ; t_{p-1}, \tau_{p-1}\right)=\frac{1}{h} \delta_{r, j}=\left\{\begin{array}{lll}
\frac{1}{h} & (r=j) & \left(\delta_{r, j}\right. \text { is } \\
0 & (r \neq j)
\end{array} \quad\right. \text { Kronecker's delta) } \\
& (p=1,2,3, \ldots)
\end{aligned}
$$

allows us to say that (A.9) is valid also for $n=0$, i. e.,

$$
u_{r}^{0}=\phi_{r}(r=1,2, \ldots, J-1) \text {. }
$$

\section{A. 2. Expression for $\boldsymbol{u}_{\boldsymbol{x}}$}

Suppose now that two functions, $v$ and $w$, satisfy the equation

$$
(\text { A. } 14)_{1} \quad\left(v_{j}^{p}\right)_{\xi \bar{\xi}}-\left(v_{j}^{p}\right)_{\bar{\tau}}=0 \quad(p=1,2,3, \ldots, n)
$$

and

$$
(\mathrm{A} .14)_{2} \quad\left(w_{j}^{p-1}\right)_{\xi \bar{\xi}}+\left(w_{j}^{p-1}\right)_{\tau}=0 \quad(p=1,2,3, \ldots, n),
$$

respectively. Multiplying (A. 14) ${ }_{1}$ by $k_{p} h w_{j}^{p-1}$ and (A. 14) ${ }_{2}$ by $k_{p} h v_{j}^{p}$ and summing up all the products, we obtain

$$
\begin{aligned}
\sum_{p=1}^{n} k_{p} \sum_{j=1}^{J-1} h\left[w_{j}^{p-1} v_{j \xi \xi}^{p}-v_{j}^{p} w_{j \xi \xi}^{p-1}\right] & \\
- & \sum_{p=1}^{n} k_{p} \sum_{j=1}^{J-1} h\left[v_{j \tau}^{p-1} w_{j}^{p-1}+v_{j}^{p} w_{j \tau}^{p}\right]=0 .
\end{aligned}
$$

Summation by part here yields

$$
\text { (A.15) } \quad \begin{gathered}
\sum_{j=1}^{J-1} h w w_{j}^{n} v_{j}^{n}=\sum_{j=1}^{J-1} h w_{j}^{0} v_{j}^{0}+\sum_{p=1}^{n} k_{p}\left[w_{J-1}^{p-1}\left(v_{J-1}^{p}\right)_{\xi}-v_{J-1}^{p}\left(w_{J-1}^{p-1}\right)_{\xi}\right] \\
-\sum_{p=1}^{n} k_{p}\left[w_{0}^{p-1} v_{0 \xi}^{p}-v_{0}^{p} w_{0 \xi}^{p-1}\right] \\
=\sum_{j=1}^{J-1} h w_{j}^{0} v_{j}^{0}+\sum_{p=1}^{n} k_{p}\left[w_{J}^{p-1} v_{J \xi}^{p}-w_{J \xi}^{p-1} v_{J}^{p}\right] \\
-\sum_{p=1}^{n} k_{p}\left[w_{0}^{p-1} v_{0 \xi}^{p}-v_{0}^{p} w_{0 \xi}^{p-1}\right] .
\end{gathered}
$$

Especially, we can take

$$
w_{j}^{p-1}=G\left(x_{r}, \xi_{j} ; t_{n}, \tau_{p-1}\right) \text { and } v_{j}^{p}=u_{j}^{p} .
$$

Then, (A. 15) becomes 


$$
\begin{aligned}
& \text { (A. 16) } u_{r}^{n}=\sum_{j=1}^{J-1} h G\left(x_{r}, \xi_{j} ; t_{n}, 0\right) \phi_{j}+\sum_{p=1}^{n} k_{p} G\left(x_{r}, 0 ; t_{n}, \tau_{p-1}\right)_{\xi} f^{p} \\
& -\sum_{p=1}^{n} k_{p} G\left(x_{r}, \xi_{j} ; t_{n}, \tau_{p-1}\right)_{\xi} g^{p} .
\end{aligned}
$$

This is an expression of a solution of the problem (A. 1) using Green's function. It also allows us to write down an expression of $u_{x}$ :

$$
\text { (A. 17) } \begin{gathered}
\left(u_{r}^{n}\right)_{x}=\sum_{j=1}^{J-1} h G\left(x_{r}, \xi_{j} ; t_{n}, 0\right)_{x} \phi_{j}+\sum_{p=1}^{n} k_{p} G\left(x_{r}, 0 ; t_{n}, \tau_{p-1}\right)_{\xi x} f^{p} \\
-\sum_{p=1}^{n} k_{p} G\left(x_{r}, \xi_{J} ; t_{n}, \tau_{p-1}\right)_{\bar{\xi} x} g^{p} .
\end{gathered}
$$

\section{A. 3. Difference Quotients of Green's Function}

We here prepare some integral expressions for difference quotients of Green's function, in order to estimate the right hand side of (A. 17). Just from definition of Green's function (A. 10), we have

$$
\text { (A. 18) } \begin{aligned}
G\left(x_{r}, \xi_{j} ; t_{n}, 0\right)_{x} & =\frac{4}{\pi h^{2}} \sum_{s=1}^{J-1} \prod_{q=1}^{n}\left(1+4 \kappa_{q} \sin ^{2} \frac{s \pi}{2 J}\right)^{-1} \sin \frac{s \pi}{2 J} \cos \left(r+\frac{1}{2}\right) \frac{s \pi}{J} \\
& \sim \frac{4}{\pi h^{2}} \int_{0}^{\pi} \prod_{q=1}^{n} \Lambda_{q}^{-1} \sin \frac{\omega}{2} \sin j \omega \cos \left(r+\frac{1}{2}\right) \omega d \omega,
\end{aligned}
$$

where $\sim$ means that its left hand side may be replaced by its right hand side for estimation for sufficiently small $h$, and

$$
\Lambda_{q}=1+4 \kappa_{q} \sin ^{2} \frac{\omega}{2} \text {. }
$$

Further, we expand the right hand side of (A. 18) as follows:

$$
\begin{gathered}
\text { (A. 20) } G\left(x_{r}, \xi_{j} ; t_{n}, 0\right)_{x} \sim \frac{4}{\pi h^{2}} \int_{0}^{\pi} \prod_{q=1}^{n} \Lambda_{q}^{-1} \sin \frac{\omega}{2} \sin j \omega\left(\cos r \omega \cos \frac{\omega}{2}\right. \\
\left.\quad-\sin r \omega \sin \frac{\omega}{2}\right) d \omega \\
=\frac{2}{\pi h^{2}} \int_{0}^{\pi} \prod_{q=1}^{n} \Lambda_{q}^{-1} \sin \omega[\sin (r+j) \omega-\sin (r-j) \omega] d \omega \\
+\frac{2}{\pi h^{2}} \int_{0}^{\pi} \prod_{q=1}^{n} \Lambda_{q}^{-1} \sin ^{2} \frac{\omega}{2}[\cos (r+j) \omega-\cos (r-j) \omega] d \omega .
\end{gathered}
$$

Similarly, we get

(A. 21) $G\left(x_{r}, 0 ; t_{n}, \tau_{p-1}\right)_{x \xi} \sim \frac{4}{\pi h^{3}} \int_{0}^{\pi} \prod_{q=p}^{n} \Lambda_{q}^{-1} \sin ^{2} \frac{\omega}{2}[\cos (\mathrm{r}+1) \omega+\cos r \omega] d \omega$ and 
(A. 22)

$$
\begin{gathered}
G\left(x_{r}, \xi_{J} ; t_{n}, \tau_{p-1}\right)_{x \bar{\xi}} \sim \frac{4}{\pi h^{3}} \int_{0}^{\pi} \prod_{q=p}^{n} \Lambda_{q}^{-1} \sin ^{2} \frac{\omega}{2}[\cos (J+r) \omega \\
+\cos (J-r-1) \omega] d \omega .
\end{gathered}
$$

Put

$$
I\left(K ; t_{n}, \tau_{p-1}\right)=\frac{2}{\pi h^{2}} \int_{0}^{\pi} \prod_{q=p}^{n} \Lambda_{q}^{-1} \sin \omega \sin K \omega d \omega
$$

and

$$
J\left(K ; t_{n}, \tau_{p-1}\right)=\frac{2}{\pi h^{3}} \int_{0}^{\pi} \prod_{q=p}^{n} \Lambda_{q}^{-1} \sin ^{2} \frac{\omega}{2} \cos K \omega d \omega .
$$

By using the symbol $I$ and $J$, we can rewrite (A.20)-(A.22) as follows:

$$
\begin{aligned}
G\left(x_{r}, \xi_{j} ; t_{n}, 0\right)_{x} & \sim I\left(r+j ; t_{n}, 0\right)-I\left(r-j ; t_{n}, 0\right) \\
& +h\left[J\left(r+j ; t_{n}, 0\right)-J\left(r-j ; t_{n}, 0\right],\right.
\end{aligned}
$$

(A. 26) $G\left(x_{r}, 0 ; t_{n}, \tau_{p-1}\right)_{x \xi} \sim 2\left[J\left(r+1 ; t_{n}, \tau_{p-1}\right)+J\left(r ; t_{n}, \tau_{p-1}\right)\right]$

and

(A. 27) $G\left(x_{r}, \xi_{J} ; t_{n}, \tau_{p-1}\right)_{x \bar{\xi}} \sim 2\left[J\left(J+r ; t_{n}, \tau_{p-1}\right)+J\left(J-r-1 ; t_{n}, \tau_{p-1}\right)\right]$.

Our next problem is to estimate the function $I$ and $J$. For it, we will prepare some lemma in the following sections.

\section{A. 4. Product of $\mathbb{1}_{q}$.}

A simple estimation from under for a product of $\Lambda_{q}$ is given as follows:

$$
\text { (A. 28) } \quad \prod_{q=p}^{n} \Lambda_{q} \geqq 1+4 \sum_{q=p}^{n} \Lambda_{q} \sin ^{2} \frac{\omega}{2}=1+\frac{4}{h^{2}}\left(t_{n}-\tau_{p-1}\right) \sin ^{2} \frac{\omega}{2} \text {. }
$$

It is, however, not sufficient for later purpose. We must prepare more sharp estimations.

Lemma A. 1 . Let $k_{q}(q=1,2, \ldots, n)$ be a sequence of positive numbers satisfying the condition

$$
\max _{q} k_{q}=k_{1}, \max _{q \neq 1} k_{q}=k_{2} \text { and } \sum_{q=1}^{n} k_{q}=t \text {. }
$$

Then, there are two kinds of partition of the number set $N=\{1,2, \ldots, n\}$, $\left\{N_{a}, N_{b}\right\}$ and $\left\{N_{\alpha}, N_{\beta}, N_{\gamma}\right\}$, such that 


$$
t_{a}=\sum_{q \in N_{a}} k_{q} \geqq \frac{1}{2}\left(t-k_{1}\right), t_{b}=\sum_{q \in N_{b}} k_{q} \geqq \frac{1}{2}\left(t-k_{1}\right)
$$

and

$$
t_{\alpha}=\sum_{q \in N_{\alpha}} k_{q} \geqq \frac{1}{3}\left(t-k_{1}-k_{2}\right), t_{\beta}=\sum_{q \in N_{\beta}} k_{q} \geqq \frac{1}{3}\left(t-k_{1}-k_{2}\right)
$$

and

$$
t_{r}=\sum_{q \in N_{a}} k_{q}<\frac{1}{3}\left(t-k_{1}-k_{2}\right) .
$$

Proof. We will first construct a partition $\left\{N_{a}, N_{b}\right\}$. Clearly, we can take a partition $\left\{1, N_{a}^{\prime}, N_{b}^{\prime}\right\}$ of $N$ such that

$$
\sum_{q \in N_{a}^{\prime}} k_{q} \leqq \sum_{q \in N_{b}^{\prime}} k_{q} \leqq \frac{t}{2}
$$

Then,

$$
\sum_{q \in N_{b}^{\prime}} k_{q} \geqq \frac{1}{2}\left(t-k_{1}\right) \text { and } \sum_{q \in N_{a}^{\prime}} k_{q}+k_{1} \geqq \frac{t}{2} .
$$

Put $N_{a}=N_{a}^{\prime}+\{1\}, N_{b}=N_{b}^{\prime}$. Such $\left\{N_{a}, N_{b}\right\}$ is a partition desired. We will next find a second partition $\left\{N_{\alpha}, N_{\beta}, N_{\gamma}\right\}$.

a) We can find a partition $\left\{N_{\alpha}^{\prime}, N_{\beta}^{\prime}, N_{\gamma}^{\prime}\right\}$ of $N^{\prime}=N-\{1,2\}$ such that

$$
t_{\alpha}^{\prime} \leqq t_{\beta}^{\prime} \leqq t_{\gamma}^{\prime} \text { and } t_{\gamma}^{\prime}-t_{\alpha}^{\prime} \leqq k_{2}
$$

where $t_{\alpha}^{\prime}=\sum_{q \in N^{\prime}} k_{q}$, etc.

In fact, let $\left\{N_{\alpha}^{0}, N_{\beta}^{0}, N_{\gamma}^{0}\right\}$ be a partition of $N^{\prime}$ such that

$$
t_{\alpha}^{0} \leqq t_{\beta}^{0} \leqq t_{r}^{0}
$$

Here and later, we put $t^{s}=\sum_{q \in N^{s}} k_{q}$, etc. $(s=0,1,2, \ldots)$. If $t_{\gamma}^{0}-t_{\alpha}^{0} \leqq k_{2}$, it is sufficient only to put $\left\{N_{\alpha}^{\prime}, N_{\beta}^{\prime}, N_{\gamma}^{\prime}\right\}=\left\{N_{\alpha}^{0}, N_{\beta}^{0}, N_{\gamma}^{0}\right\}$. Otherwise, we take off an element from $N_{\gamma}^{0}$, and call the remained $N_{\gamma}^{1}$. Adding the element to $N_{\alpha}^{0}$, we call thè result $N_{\alpha}^{1}$. Next, exchange the name of suffix, $\alpha, \beta, \gamma$ in order that $t_{\alpha}^{1} \leqq t_{\beta}^{1} \leqq t_{\gamma}^{1}$. We repeat such procedure to get a sequence of partition, $\left\{N_{\alpha}^{s}, N_{\beta}^{s}, N_{\gamma}^{s}\right\} \quad(s=1,2, \ldots)$ such that $t_{\gamma}^{s}-t_{\alpha}^{s}<t_{\gamma}^{s-1}-t_{\alpha}^{s-1}$, and finally to get $\left\{N_{\alpha}^{\prime}, N_{\beta}^{\prime}, N_{\gamma}^{\prime}\right\}$ desired after a finite number of steps.

b) Put

$$
N_{\alpha}=1+N_{\alpha}^{\prime}, N_{\beta}=2+N_{\beta}^{\prime} \text { and } N_{r}=N_{\gamma}^{\prime} .
$$

This is a partition desired. In fact, since $t_{\alpha}^{\prime}+t_{\beta}^{\prime}+t_{\gamma}^{\prime}=t-k_{1}-k_{2}$ and 
$t_{\gamma}^{\prime}=t_{r}$, we have

$$
t_{r} \geqq \frac{1}{3}\left(t-k_{1}-k_{2}\right), t_{\alpha}=t_{\alpha}^{\prime}+k_{1} \geqq t_{\gamma} \text { and } t_{\beta}=t_{\beta}^{\prime}+k_{2} \geqq t_{\gamma}
$$

due to (A. 29). Thus, we have proved Lemma A. 1 .

Lemma A.2. Assume that there is a constant $\mu(0<\mu<1)$ such that (A. 30)

$$
\min _{p \leqq q \leqq n} k_{q}>\mu \max _{p \leqq q \leqq n} k_{q}
$$

Then, the iequality

(A. 31) $\prod_{q=p}^{n} \Lambda_{q}>\left[1+\frac{\mu_{1}}{h^{2}}\left(t_{n}-\tau_{p-1}\right) \sin ^{2} \frac{\omega}{2}\right]^{2} \quad($ for $p \leqq n-1)$

and

(A. 32) $\quad \prod_{q=p}^{n} \Lambda_{q}>\left[1+\frac{\mu_{2}}{h^{2}}\left(t_{n}-\tau_{p-1}\right) \sin ^{2} \frac{\omega}{2}\right]^{3} \quad($ for $p \leqq n-2)$

hold, where $\mu_{1}=\mu$ and $\mu_{2}=\frac{4}{9} \mu$.

Proof. In order to show (A.31), we use a partition $\left\{N_{a}, N_{b}\right\}$ of $N=\{p, p+1, \ldots, n\}$, as shown in Lemma A. 1 . Then, we have

$$
\begin{aligned}
\prod_{q=p}^{n} \Lambda_{q} & =\prod_{q \in N_{a}} \Lambda_{q} \prod_{r \in N_{b}} \Lambda_{r} \\
& \geqq\left[1+\frac{2}{h^{2}}\left(t_{n}-\tau_{p-1}-\bar{k}\right) \sin ^{2} \frac{\omega}{2}\right]^{2},
\end{aligned}
$$

where $\bar{k}=\max _{q \in N_{a}} k_{q}$. From the assumption (A. 30), it follows that

$$
\begin{gathered}
t_{n}-\tau_{p-1}-\bar{k} \geqq(n-p) \min _{p \leqq q \leqq n} k_{q} \geqq\left(1-\frac{1}{n-p+1}\right)(n-p+1) \mu \bar{k} \\
\geqq \frac{\mu}{2}\left(t_{n}-\tau_{p-1}\right) .
\end{gathered}
$$

Therefore, we get (A.31) by combining the last two relations.

In order to show (A. 32), we apply a partition $\left\{N_{\alpha}, N_{\beta}, N_{\gamma}\right\}$ of $N=\{p, p+1, \ldots, n\}$, as shown in Lemma A. 1. Then,

$$
\begin{aligned}
\prod_{q=p}^{n} \Lambda_{q} & =\prod_{q \in N_{\alpha}} \Lambda_{q} \underset{r \in N_{\beta}}{ } \Lambda_{r} \prod_{s \in N_{\gamma}} \Lambda_{s} \\
& \geqq\left[1+\frac{4}{3 h^{2}}\left(t_{n}-\tau_{p-1}-\tilde{k}\right) \sin ^{2} \frac{\omega}{2}\right]^{3}
\end{aligned}
$$

where $\tilde{k}$ is the sum of the most $(\bar{k})$ and the second taken from 
$\left\{k_{q}, q=p, p+1, \ldots, n\right\}$. The condition $(\mathrm{A}, 30)$ assures that

$$
\begin{gathered}
t_{n}-\tau_{p-1}-\tilde{k} \geqq(n-p-1) \min _{p \leqq q \leqq n} k_{q} \geqq\left(1-\frac{2}{n-p+1}\right)(n-p+1) \mu \bar{k} \\
\geqq \frac{\mu}{3}\left(t_{n}-\tau_{p-1}\right) .
\end{gathered}
$$

Therefore

$$
\prod_{q=p}^{n} \Lambda_{q} \geqq\left[1+\frac{4 \mu}{9 h^{2}}\left(t_{n}-\tau_{p-1}\right) \sin ^{2} \frac{\omega}{2}\right]^{3} .
$$

This is not but (A. 32).

\section{A. 5. Some Integration Formulae}

Lemma A. 3. Let $\alpha, \beta, \gamma$ and $\delta$ be positive constants. Then

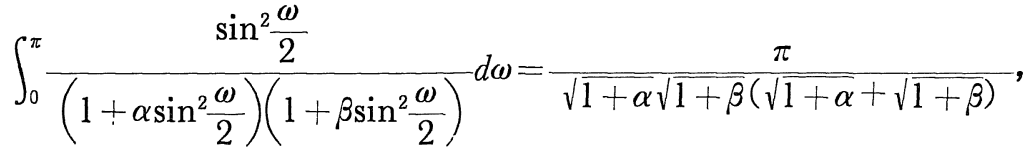

(A. 34)

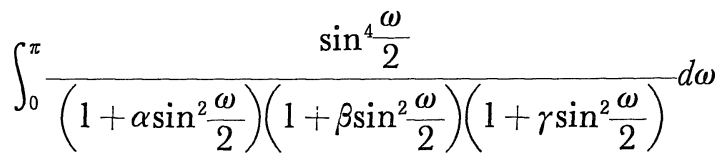

$$
=\frac{\pi(\sqrt{1+\alpha}+\sqrt{1+\beta}+\sqrt{1+\gamma})}{\sqrt{1+\alpha} \sqrt{1+\beta} \sqrt{1+\gamma}(\sqrt{1+\alpha}+\sqrt{1+\beta})(\sqrt{1+\beta}+\sqrt{1+\gamma})(\sqrt{1+\gamma}+\sqrt{1+\alpha})}
$$

and

(A. 35)

$$
\int_{0}^{\pi} \frac{\sin ^{6} \frac{\omega}{2}}{\left(1+\alpha \sin ^{2} \frac{\omega}{2}\right)\left(1+\beta \sin ^{2} \frac{\omega}{2}\right)\left(1+\gamma \sin ^{2} \frac{\omega}{2}\right)\left(1+\delta \sin ^{2} \frac{\omega}{2}\right)} d \omega=\frac{\pi A}{B},
$$

where

$$
\begin{aligned}
A= & (1+\alpha)(\sqrt{1+\beta}+\sqrt{1+\gamma}+\sqrt{1+\delta})+(1+\beta)(\sqrt{1+\gamma}+\sqrt{1+\delta}+\sqrt{1+\alpha}) \\
+ & (1+\gamma)(\sqrt{1+\delta}+\sqrt{1+\alpha}+\sqrt{1+\beta})+(1+\delta)(\sqrt{1+\alpha}+\sqrt{1+\beta}+\sqrt{1+\gamma}) \\
+ & 2(\sqrt{1+\alpha} \sqrt{1+\gamma} \sqrt{1+\delta}+\sqrt{1+\gamma} \sqrt{1+\delta} \sqrt{1+\alpha}+\sqrt{1+\delta} \sqrt{1+\alpha} \sqrt{1+\beta} \\
& +\sqrt{1+\alpha} \sqrt{1+\beta} \sqrt{1+\gamma})
\end{aligned}
$$

and 


$$
\begin{aligned}
B= & \sqrt{1+\alpha} \sqrt{1+\beta} \sqrt{1+\gamma} \sqrt{1+\delta}(\sqrt{1+\alpha}+\sqrt{1+\beta})(\sqrt{1+\alpha}+\sqrt{1+\gamma})(\sqrt{1+\alpha} \\
& +\sqrt{1+\delta})(\sqrt{1+\beta}+\sqrt{1+\gamma})(\sqrt{1+\beta}+\sqrt{1+\delta})(\sqrt{1+\gamma}+\sqrt{1+\delta}) .
\end{aligned}
$$

Its proof can be done by introducing a new argument $\eta$,

$$
\eta=\left(\frac{\sin ^{2} \frac{\omega}{2}}{1-\sin ^{2} \frac{\omega}{2}}\right)^{1 / 2},
$$

and practising residue calculations in $\eta_{0}$ Its details are omitted.

\section{A.6. Estimation of $\mathbb{I}\left(\mathbb{K} ; \tau_{n}, \tau_{p-1}\right)$}

Lemma A.4. When $p \leqq n$,

$$
\left|I\left(K ; t_{n}, \tau_{p-1}\right)\right|<\frac{C}{\sqrt{t_{n}-\tau_{p-1}}\left(\sqrt{t_{n}-\tau_{p-1}}+|K| h\right)},
$$

where $G$ is a constant independent of $t_{n}, \tau_{p-1}, K, h$ and $\left\{k_{n}\right\}$.

Proof. When $K=0$, it is trivial, since $I\left(0 ; t_{n}, \tau_{p-1}\right)=0$. In general,

$$
I\left(-K ; t_{n}, \tau_{p-1}\right)=-I\left(K ; t_{n}, \tau_{p-1}\right)
$$

and hence

$$
\left|I\left(-K ; t_{n}, \tau_{p-1}\right)\right|=\left|I\left(K ; t_{n}, \tau_{p-1}\right)\right| \text { 。 }
$$

So, it is sufficient only to consider the case that $K$ is a positive constant. Put

$$
\text { (A. 37) } \quad I\left(K ; t_{n}, \tau_{p-1}\right)=I_{1}\left(K ; t_{n}, \tau_{p-1}\right)+I_{2}\left(K ; t_{n}, \tau_{p-1}\right) \text {, }
$$

where

$(\mathrm{A} .38)_{1} \quad I_{1}\left(K ; t_{n}, \tau_{p-1}\right)=\frac{2}{\pi h^{2}} \int_{0}^{\frac{\pi}{K}} \prod_{q=p}^{n} \Lambda_{q}^{-1} \sin \omega \sin K \omega d \omega$

and

$$
(\mathrm{A} .38)_{2} \quad I_{2}\left(K ; t_{n}, \tau_{p-1}\right)=\frac{2}{\pi h^{2}} \int_{\frac{\pi}{K}}^{\pi} \prod_{q=p}^{n} \Lambda_{q}^{-1} \sin \omega \sin K \omega d \omega .
$$

We first consider $I_{1}\left(K ; t_{n}, \tau_{p-1}\right)$. Notice that

$$
|\sin K \omega|=2\left|\sin \frac{K \omega}{2}\right| \circ\left|\cos \frac{K \omega}{2}\right|
$$


and

$$
\begin{aligned}
& \left|\sin \frac{K \omega}{2}\right| \leqq \frac{K \omega}{2} \leqq \frac{K \pi}{2} \sin \frac{\omega}{2} \text { for } 0 \leqq \omega \leqq \pi \\
& \left|\cos \frac{K \omega}{2}\right|=\left|1-2 \sin ^{2} \frac{K \omega}{4}\right|<\frac{1}{1+2 \sin ^{2} \frac{K \omega}{4}} \\
& <\frac{1}{1+\frac{K^{2} \omega^{2}}{2 \pi^{2}}}<\frac{1}{1+\frac{2 K^{2}}{\pi^{2}} \sin ^{2} \frac{\omega}{2}} \quad \text { for } 0 \leqq \omega \leqq \frac{\pi}{K}
\end{aligned}
$$

So, we have

$$
\left|I_{1}\left(K ; t_{n}, \tau_{p-1}\right)\right|<\frac{4}{h^{2}} \int_{0}^{\frac{\pi}{K}} \prod_{q=p}^{n} \Lambda_{q}^{-1} \frac{K \sin ^{2} \frac{\omega}{2} \cos \frac{\omega}{2}}{1+\frac{2 K^{2}}{\pi^{2}} \sin ^{2} \frac{\omega}{2}} d \omega .
$$

Applying (A. 28) on the last integrand yields

$$
\mid I_{1}\left(K ; t_{n}, \tau_{p-1} \mid<\frac{4 K}{h^{2}} \int_{0}^{\pi} \frac{\sin ^{2} \frac{\omega}{2}}{\left(1+\frac{2 K^{2}}{\pi^{2}} \sin ^{2} \frac{\omega}{2}\right)\left[1+\frac{4}{h^{2}}\left(t_{n}-\tau_{p-1}\right) \sin ^{2} \frac{\omega}{2}\right]} d \omega .\right.
$$

Further, by using (A. 33), we obtain

$$
\begin{aligned}
& \text { (A. 39) }\left|I_{1}\left(K ; t_{n}, \tau_{p-1}\right)\right| \\
& <\frac{4 K}{h^{2}} \frac{1}{\left.\sqrt{1+\frac{4}{h^{2}}\left(t_{n}-\tau_{p-1}\right)} \sqrt{1+\frac{2 K^{2}}{\pi^{2}}\left[\sqrt{1+\frac{4}{h^{2}}\left(t_{n}-\tau_{p-1}\right)}+\sqrt{1+\frac{2 K^{2}}{\pi^{2}}}\right.}\right]} \\
& <\frac{C}{\sqrt{t_{n}-\tau_{p-1}}\left(\sqrt{t_{n}-\tau_{p-1}}+K h\right)}
\end{aligned}
$$

with an appropriate constant $C$.

We next consider $I_{2}\left(K ; t_{n}, \tau_{p-1}\right)$. Through integration by part, we get

$$
\begin{aligned}
& I_{2}\left(K ; t_{n}, \tau_{p-1}\right)=\frac{4}{\pi h^{2}} \int_{\frac{\pi}{K}}^{\pi} \prod_{q=p}^{n} \Lambda_{q}^{-1} \sin \omega \cos \frac{K \omega}{2} \sin \frac{K \omega}{2} d \omega \\
& =\frac{8}{\pi K h^{2}}\left[\int_{\frac{\pi}{K}}^{\pi} \prod_{q=p}^{n} \Lambda_{q}^{-1} \cos \omega \cos ^{2} \frac{K \omega}{2} d \omega\right. \\
& \left.\quad-\int_{\frac{\pi}{K}}^{\pi} \prod_{q=p}^{n} \Lambda_{q}^{-1} \sin \omega \cos ^{2} \frac{K \omega}{2} \sum_{q=p}^{n} \frac{4 \kappa_{q} \sin \frac{\omega}{2} \cos \frac{\omega}{2}}{1+4 \kappa_{q} \sin ^{2} \frac{\omega}{2}} d \omega\right]-I_{2}\left(K ; t_{n}, \tau_{p-1}\right) .
\end{aligned}
$$


Hence

$$
\text { (A. 40) } \quad I_{2}\left(K ; t_{n}, \tau_{p-1}\right)=I_{21}\left(K ; t_{n}, \tau_{p-1}\right)+I_{22}\left(K ; t_{n}, \tau_{p-1}\right) \text {, }
$$

where

$$
I_{21}\left(K ; t_{n}, \tau_{p-1}\right)=\frac{4}{\pi K h^{2}} \int_{\frac{\pi}{K}}^{\pi} \prod_{q=p}^{n} \Lambda_{q}^{-1} \cos \omega \cos ^{2} \frac{K \omega}{2} d \omega
$$

and

(A. 42) $\quad I_{22}\left(K ; t_{n}, \tau_{p-1}\right)$

$$
=-\frac{8}{\pi K h^{2}} \int_{\frac{\pi}{K}}^{\pi} \prod_{q=p}^{n} \Lambda_{q}^{-1} \cos ^{2} \frac{\omega}{2} \cos ^{2} \frac{K \omega}{2} \sum_{q=p}^{n} \frac{4 \kappa_{q} \sin ^{2} \frac{\omega}{2}}{1+4 \kappa_{q} \sin ^{2} \frac{\omega}{2}} d \omega .
$$

Let us consider two cases, i) $p=n$ and ii) $p<n$, separately. When $p=n$,

$$
\begin{aligned}
& I_{2}\left(K ; t_{n}, \tau_{n-1}\right) \\
& =\frac{4}{\pi K h^{2}}\left\{\int_{\frac{\pi}{K}}^{\pi}\left[\frac{\cos \omega \cos ^{2} \frac{K \omega}{2}}{1+4 \kappa_{n} \sin ^{2} \frac{\omega}{2}}-\frac{8 \kappa_{n} \cos ^{2} \frac{\omega}{2} \cos ^{2} \frac{K \omega}{2} \sin ^{2} \frac{\omega}{2}}{\left(1+4 \kappa_{n} \sin ^{2} \frac{\omega}{2}\right)^{2}}\right] d \omega\right\} \\
& =\frac{4}{\pi K h^{2}} \int_{\frac{\pi}{K}}^{\pi} \frac{\cos ^{2} \frac{K \omega}{2} \cos \omega-4 \kappa_{n} \sin ^{2} \frac{\omega}{2} \cos ^{2} \frac{K \omega}{2}}{\left(1+4 \kappa_{n} \sin ^{2} \frac{\omega}{2}\right)^{2}} d \omega,
\end{aligned}
$$

and hence

$$
\left|I_{2}\left(K ; t_{n}, \tau_{n-1}\right)\right|<\frac{4}{\pi K h^{2}} \int_{\frac{\pi}{K}}^{\pi} \frac{d \omega}{1+4 \kappa_{n} \sin ^{2} \frac{\omega}{2}} .
$$

Since $K^{2} \sin ^{2} \frac{\omega}{2}>\frac{K^{2} \omega^{2}}{\pi^{2}}>1$ for $\omega>\frac{\pi}{K}$, we have

$$
\begin{aligned}
\int_{\frac{\pi}{K}}^{\pi} \frac{d \omega}{1+4 \kappa_{n} \sin ^{2} \frac{\omega}{2}} & <2 K^{2} \int_{0}^{\pi} \frac{\sin ^{2} \frac{\omega}{2}}{\left(1+K^{2} \sin ^{2} \frac{\omega}{2}\right)\left(1+4 \kappa_{n} \sin ^{2} \frac{\omega}{2}\right)} d \omega \\
= & \frac{2 K^{2} \pi}{\sqrt{1+K^{2}} \sqrt{1+4 \kappa_{n}}\left(\sqrt{1+K^{2}}+\sqrt{1+4 \kappa_{n}}\right)} .
\end{aligned}
$$

Here, the last equality follows from (A.33). Therefore

$$
\text { (A. 43)' } \quad\left|I_{2}\left(K ; t_{n}, \tau_{n-1}\right)\right|<\frac{C K}{\sqrt{1+K^{2}}\left(K h+\sqrt{k_{n}}\right) \sqrt{k_{n}}}
$$


or

$$
\left|I_{2}\left(K ; t_{n}, \tau_{n-1}\right)\right|<\frac{C}{\left(K h+\sqrt{t_{n}-\tau_{n-1}}\right) \sqrt{t_{n}-\tau_{n-1}}} .
$$

Also here and later, $C$ is a constant selected appropriately. By applying (A.43) and (A.39) upon (A.37), we have the desired estimation (A.36) for $p=n$. When $p<n$, we consider $I_{21}$ and $I_{22}$ separately. Notice again that

$$
\text { (A. 44) } \quad K^{2} \sin ^{2} \frac{\omega}{2}>\frac{K^{2} \omega^{2}}{\pi^{2}}>1 \quad \text { for } \frac{\pi}{K}<\omega<\pi \text {. }
$$

We then have, by (A.28) and (A.33),

$$
\begin{gathered}
\left|I_{21}\left(K ; t_{n}, \tau_{p-1}\right)\right|<\frac{8 K}{\pi h^{2}} \int_{0}^{\pi} \frac{\sin ^{2} \frac{\omega}{2}}{\left(1+K^{2} \sin ^{2} \frac{\omega}{2}\right)\left[1+\frac{4}{h^{2}}\left(t_{n}-\tau_{p-1}\right) \sin ^{2} \frac{\omega}{2}\right]} d \omega \\
=\frac{8 K}{h^{2} \sqrt{1+K^{2}} \sqrt{1+\frac{4}{h^{2}}\left(t_{n}-\tau_{p-1}\right)\left[\sqrt{1+K^{2}}+\sqrt{1+\frac{4}{h^{2}}\left(t_{n}-\tau_{p-1}\right)}\right]}},
\end{gathered}
$$

and further

$$
(\text { A. } 45)^{\prime} \quad\left|I_{21}\left(K ; t_{n}, \tau_{p-1}\right)\right|<\frac{C K}{\left.\sqrt{1+K^{2} \sqrt{t_{n}-\tau_{p-1}}\left(\sqrt{t_{n}-\tau_{p-1}}\right.}+K h\right)},
$$

$$
\mid I_{21}\left(K ; t_{n}, \tau_{p-1}\right):<\frac{C}{\sqrt{\overline{t_{n}-\tau_{p-1}}\left(\sqrt{t_{n}-\tau_{p-1}}+K h\right)}} .
$$

We next consider $I_{22}$, and apply (A.31), (A.44) and successively. Then

$$
\begin{aligned}
& \left|I_{22}\left(K ; t_{n}, \tau_{p-1}\right)\right|<\frac{32}{\pi K h^{2}} \sum_{q=p}^{n} \kappa_{q} \int_{\frac{\pi}{K}}^{\pi} \prod_{q=p}^{n} \Lambda_{q}^{-1} \sin ^{2} \frac{\omega}{2} d \omega \\
& <\frac{64 K}{h^{2}} \sum_{q=p}^{n} \kappa_{q} \int_{0}^{\pi} \frac{\sin ^{4} \frac{\omega}{2}}{\left(1+K^{2} \sin ^{2} \frac{\omega}{2}\right)\left[1+\frac{\mu}{h^{2}}\left(t_{n}-\tau_{p-1}\right) \sin ^{2} \frac{\omega}{2}\right]^{2}} d \omega \\
& =\frac{32 K\left(t_{n}-\tau_{p-1}\right)\left[2 \sqrt{1+\frac{\mu}{h^{2}}\left(t_{n}-\tau_{p-1}\right)}+\sqrt{1+K^{2}}\right]}{h^{4}\left[1+\frac{\mu}{h^{2}}\left(t_{n}-\tau_{p-1}\right)\right]^{3 / 2} \sqrt{1+K^{2}}\left[\sqrt{1+\frac{\mu}{h^{2}}\left(t_{n}-\tau_{p-1}\right)}+\sqrt{1+K^{2}}\right]^{2}} .
\end{aligned}
$$

Hence 
$(\mathrm{A} .46)^{\prime} \quad\left|I_{22}\left(K ; t_{n}, \tau_{p-1}\right)\right|<\frac{C K}{\sqrt{t_{n}-\tau_{p-1}}\left(\sqrt{t_{n}-\tau_{p-1}}+K h\right) \sqrt{1+K^{2}}}$

or

(A. 46) $\left|I_{22}\left(K ; t_{n}, \tau_{p-1}\right)\right|<\frac{G}{\sqrt{t_{n}-\tau_{p-1}}\left[\sqrt{t_{n}-\tau_{p-1}}+K h\right]}$.

By applying (A. 45)' and (A. 46)', or (A.45) and (A.46) upon (A. 40), we get

$(\mathrm{A} .47)^{\prime} \quad\left|I_{2}\left(K ; t_{n}, \tau_{p-1}\right)\right|<\frac{C K}{\sqrt{t_{n}-\tau_{p-1}}\left(\sqrt{t_{n}-\tau_{p-1}}+K h\right) \sqrt{1+K^{2}}}$,

(A. 47) $\left|I_{2}\left(K ; t_{n}, \tau_{p-1}\right)\right|<\frac{C}{\sqrt{t_{n}-\tau_{p-1}}\left(\sqrt{t_{n}-\tau_{p-1}}+K h\right)}$.

Finally, by applying (A.39) and (A.47) on (A.37), we also have the desired estimation (A.36) for $p<n$. Thus, we have proved Lemma A. 4.

\section{A. 7. Estimation of $\mathscr{I}\left(\mathbb{R} ; \mathbb{t}_{n}, \tau_{p-1}\right)$}

Lemma A. 5. When $p \leqq n$,

(A. 48)

$$
\left|J\left(K ; t_{n}, \tau_{p-1}\right)\right|<\frac{C}{h \sqrt{1+K^{2}} \sqrt{t_{n}-\tau_{p-1}}\left(\sqrt{t_{n}-\tau_{p-1}}+|K| h\right)} .
$$

Proof. When $K=0$, we apply (A.28) and (A.33) successively.

$$
\begin{aligned}
J\left(0 ; t_{n}, \tau_{p-1}\right) & =\frac{2}{\pi h^{3}} \int_{0}^{\pi} \prod_{q=p}^{n} \Lambda_{q}^{-1} \sin ^{2} \frac{\omega}{2} d \omega \\
& <\frac{2}{\pi h^{3}} \int_{0}^{\pi} \frac{\sin ^{2} \frac{\omega}{2}}{1+\frac{4}{h^{2}}\left(t_{n}-\tau_{p-1}\right) \sin ^{2} \frac{\omega}{2}} d \omega \\
& =\frac{2}{h^{3} \sqrt{1+\frac{4}{h^{2}}\left(t_{n}-\tau_{p-1}\right)}\left[1+\sqrt{1+\frac{4}{h^{2}}\left(t_{n}-\tau_{p-1}\right)}\right]} .
\end{aligned}
$$

Hence

$$
J\left(0 ; t_{n}, \tau_{p-1}\right)<\frac{G}{h\left(t_{n}-\tau_{p-1}\right)} .
$$

This shows that (A. 48) is valid when $K=0$.

Let us next consider the general case $(K \neq 0)$. Since $J(-K$; 
$\left.t_{n}, \tau_{p-1}\right)=J\left(K ; t_{n}, \tau_{p-1}\right)$, it is sufficient for the proof only to consider every case of a positive number $K$. Put

$$
\text { (A. 49) } J\left(K ; t_{n}, \tau_{p-1}\right)=J_{1}\left(K ; t_{n}, \tau_{p-1}\right)+J_{2}\left(K ; t_{n}, \tau_{p-1}\right) \text {, }
$$

where

(A. 50) $J_{1}\left(K ; t_{n}, \tau_{p-1}\right)=\frac{2}{\pi h^{3}} \int_{0}^{\frac{\pi}{K}} \prod_{q=p}^{n} \Lambda_{q}^{-1} \sin ^{2} \frac{\omega}{2} \cos K \omega d \omega$

and

(A. 51) $J_{2}\left(K ; t_{n}, \tau_{p-1}\right)=\frac{2}{\pi h^{3}} \int_{\frac{\pi}{K}}^{\pi} \prod_{q=p}^{n} \Lambda_{q}^{-1} \sin ^{2} \frac{\omega}{2} \cos K \omega d \omega$.

We first consider $J_{1}$. By applying the inequality

(A. 52) $1<\frac{2}{1+\frac{K^{2} \omega^{2}}{\pi^{2}}}<\frac{2}{1+\frac{4 K^{2}}{\pi^{2}} \sin ^{2} \frac{\omega}{2}}$ for $0<\omega<\frac{\pi}{K}$,

(A. 28) and (A. 33) successively, we get

$$
\begin{aligned}
& \left|J_{1}\left(K ; t_{n}, \tau_{p-1}\right)\right|<\frac{4}{\pi h^{3}} \int_{0}^{\pi} \frac{\sin ^{2} \frac{\omega}{2}}{\left(1+\frac{4 K^{2}}{\pi^{2}} \sin ^{2} \frac{\omega}{2}\right)\left[1+\frac{4}{h^{2}}\left(t_{n}-\tau_{p-1}\right) \sin ^{2} \frac{\omega}{2}\right]} d \omega \\
& =\frac{4}{h^{3} \sqrt{1+\frac{4}{h^{2}}\left(t_{n}-\tau_{p-1}\right)} \sqrt{1+\frac{4 K^{2}}{\pi^{2}}}\left[\sqrt{1+\frac{4}{h^{2}}\left(t_{n}-\tau_{p-1}\right)}+\sqrt{1+\frac{4 K^{2}}{\pi^{2}}}\right]} .
\end{aligned}
$$

Hence

$$
\text { (A. 53) }\left|J_{1}\left(K ; t_{n}, \tau_{p-1}\right)\right|<\frac{C}{h \sqrt{1+K^{2}} \sqrt{t_{n}-\tau_{p-1}}\left(\sqrt{t_{n}-\tau_{p-1}}+K h\right)} .
$$

We next consider $J_{2}$. It is easily found by integration by part that (A. 54) $J_{2}\left(K ; t_{n}, \tau_{p-1}\right)=J_{21}\left(K ; t_{n}, \tau_{p-1}\right)+J_{22}\left(K ; t_{n}, \tau_{p-1}\right)$, where

$$
\text { (A. 55) } J_{21}\left(K ; t_{n}, \tau_{p-1}\right)=-\frac{1}{\pi K h^{3}} \int_{\frac{\pi}{K}}^{\pi} \prod_{q=p}^{n} \Lambda_{q}^{-1} \sin \omega \sin K \omega d \omega
$$

and

(A. 56) $J_{22}\left(K ; t_{n}, \tau_{p-1}\right)$

$$
=\frac{2}{\pi K h^{3}} \int_{\frac{\pi}{K}}^{\pi} \prod_{q=p}^{n} \Lambda_{q}^{-1} \sin ^{2} \frac{\omega}{2} \sin K \omega \sum_{q=p}^{n} \frac{4 \kappa_{q} \sin \frac{\omega}{2} \cos \frac{\omega}{2}}{1+4 \kappa_{q} \sin ^{2} \frac{\omega}{2}} d \omega .
$$


We first deal with tha case of $p=n$ exclusively.

$$
\begin{aligned}
J_{2}\left(K ; t_{n}, \tau_{n-1}\right)= & -\frac{1}{\pi K h^{3}} \int_{\frac{\pi}{K}}^{\pi} \frac{\sin \omega \sin K \omega}{1+4 \kappa_{n} \sin ^{2} \frac{\omega}{2}} d \omega \\
& +\frac{2}{\pi K h^{3}} \int_{\frac{\pi}{K}}^{\pi} \frac{\sin ^{2} \frac{\omega}{2} \sin K \omega}{1+4 \kappa_{n} \sin ^{2} \frac{\omega}{2}} \cdot \frac{4 \kappa_{n} \sin \frac{\omega}{2} \cos \frac{\omega}{2}}{1+4 \kappa_{n} \sin ^{2} \frac{\omega}{2}} d \omega \\
= & -\frac{1}{K h^{3}} \int_{\frac{\pi}{K}}^{\pi} \frac{\sin K \omega \sin \frac{\omega}{\left(1+4 \kappa_{n} \sin ^{2} \frac{\omega}{2}\right)^{2}} d \omega .}{}
\end{aligned}
$$

The last integral can be estimated as done for $I_{2}\left(K ; t_{n}, \tau_{p-1}\right)$ (see $\left.(\text { A. } 43)^{\prime}\right)$. So, we have

$$
\left|J_{2}\left(K ; t_{n}, \tau_{n-1}\right)\right|<\frac{C}{h \sqrt{1+K^{2}} \sqrt{k_{n}}\left(\sqrt{k_{n}}+K h\right)} .
$$

Hence and from (A.52), it follows that the inequality (A.47) is valid for $p=n$ and $K \neq 0$.

We notice for the general case, $p<n$, that $J_{21}\left(K ; t_{n}, \tau_{p-1}\right)$ can be estimated in such a way as for $I_{2}\left(K ; t_{n}, \tau_{p-1}\right)$ (see $\left.(\mathrm{A} .47)^{\prime}\right)$. Then (A. 57) $\left|J_{21}\left(K ; t_{n}, \tau_{p-1}\right)\right|<\frac{C}{h \sqrt{1+K^{2}} \sqrt{t_{n}-\tau_{p-1}}\left[\sqrt{t_{n}-\tau_{p-1}}+K h\right]}$.

We are to go to estimate $J_{22}$.

(A. 58) $\left|J_{22}\left(K ; t_{n}, \tau_{p-1}\right)\right|$

$$
<\frac{8\left(t_{n}-\tau_{p-1}\right)}{\pi K h^{5}}\left|\int_{\frac{\pi}{K}}^{\pi} \prod_{q=p}^{n} \Lambda_{q}^{-1} \sin ^{2} \frac{\omega}{2} \sin \omega \sin \frac{K \omega}{2} \cos \frac{K \omega}{2} d \omega\right| .
$$

Consider the last integral. Put

$$
\text { (A. 59) } L\left(K ; t_{n}, \tau_{p-1}\right)=\int_{\frac{\pi}{K}}^{\pi} \prod_{q=p}^{n} \Lambda_{q}^{-1} \sin ^{2} \frac{\omega}{2} \sin \omega \sin \frac{K \omega}{2} \cos \frac{K \omega}{2} d \omega \text {. }
$$

Through integration by part, we get

$$
\begin{aligned}
& L\left(K ; t_{n}, \tau_{p-1}\right) \\
& \quad=\frac{2}{K} \int_{\frac{\pi}{K}}^{\pi} \prod_{q=p}^{n} \Lambda_{q}^{-1}\left(\sin \frac{\omega}{2} \cos \frac{\omega}{2} \sin \omega \cos ^{2} \frac{K \omega}{2}+\sin ^{2} \frac{\omega}{2} \cos \omega \cos ^{2} \frac{K \omega}{2}\right) d \omega
\end{aligned}
$$




$$
\begin{aligned}
& -\frac{2}{K} \int_{\frac{\pi}{K}}^{\pi} \prod_{q=p}^{n} \Lambda_{q}^{-1} \sin ^{2} \frac{\omega}{2} \sin \omega \cos ^{2} \frac{K \omega}{2} \sum_{q=p}^{n} \frac{4 \kappa_{q} \sin \frac{\omega}{2} \cos \frac{\omega}{2}}{1+4 \kappa_{q} \sin ^{2} \frac{\omega}{2}} d \omega \\
& -L\left(K ; t_{n}, \tau_{p-1}\right) .
\end{aligned}
$$

Hence

(A. 60$) L\left(K ; t_{n}, \tau_{p-1}\right)$

$$
\begin{aligned}
& =\frac{1}{K} \int_{\frac{\pi}{K}}^{\pi} \prod_{q=p}^{n} \Lambda_{q}^{-1}\left[\sin \frac{\omega}{2} \cos \frac{\omega}{2} \cos ^{2} \frac{K \omega}{2}+\sin ^{2} \frac{\omega}{2} \cos \omega \cos ^{2} \frac{K \omega}{2}\right] d \omega \\
& -\frac{1}{K} \int_{\frac{\pi}{K}}^{\pi} \prod_{q=p}^{n} \Lambda_{q}^{-1} \sin ^{2} \frac{\omega}{2} \sin \omega \cos ^{2} \frac{K \omega}{2} \sum_{q=p}^{n} \frac{4 \kappa_{q} \sin \frac{\omega}{2} \cos \frac{\omega}{2}}{1+4 \kappa_{q} \sin ^{2} \frac{\omega}{2}} d \omega .
\end{aligned}
$$

We first consider the case of $p=n-1$, exclusively.

$$
\begin{aligned}
& L\left(K ; t_{n}, \tau_{n-2}\right) \\
& =\frac{1}{K} \int_{\frac{\pi}{K}}^{\pi} \Lambda_{n-1}^{-1} \Lambda_{n}^{-1}\left[2 \sin ^{2} \frac{\omega}{2} \cos ^{2} \frac{\omega}{2} \cos ^{2} \frac{K \omega}{2}\right. \\
& \left.+\sin ^{2} \frac{\omega}{2}\left(2 \cos ^{2} \frac{\omega}{2}-1\right) \cos ^{2} \frac{K \omega}{2}\right] d \omega \\
& -\frac{2}{K} \int_{\frac{\pi}{K}}^{\pi} \Lambda_{n-1}^{-1} \Lambda_{n}^{-1} \sin ^{2} \frac{\omega}{2} \cos ^{2} \frac{\omega}{2} \cos ^{2} \frac{K \omega}{2} \sum_{q=n-1}^{n} \frac{4 \kappa_{q} \sin ^{2} \frac{\omega}{2}}{1+4 \kappa_{q} \sin ^{2} \frac{\omega}{2}} d \omega \\
& =-\frac{1}{K} \int_{\frac{\pi}{K}}^{\pi} \Lambda_{n-1}^{-1} \Lambda_{n}^{-1} \sin ^{2} \frac{\omega}{2} \cos ^{2} \frac{\omega}{2} d \omega \\
& +\frac{2}{K} \int_{\frac{\pi}{K}}^{\pi} \Lambda_{n-1}^{-1} \Lambda_{n}^{-1} \sin ^{2} \frac{\omega}{2} \cos ^{2} \frac{\omega}{2} \cos ^{2} \frac{K \omega}{2} \sum_{q=n-1}^{n} \frac{1}{1+4 \kappa_{q} \sin ^{2} \frac{\omega}{2}} d \omega .
\end{aligned}
$$

We hence have, by using (A. 31), (A. 44) and (A. 34),

$$
\begin{aligned}
\left|L\left(K ; t_{n}, \tau_{n-2}\right)\right| & <\frac{5}{K} \int_{\frac{\pi}{K}}^{\pi} \Lambda_{n-1}^{-1} \Lambda_{n}^{-1} \sin ^{2} \frac{\omega}{2} d \omega \\
& <\frac{{ }^{8} 5-}{K} \int_{\frac{\pi}{K}}^{\pi} \frac{\sin ^{2} \frac{\omega}{2}}{\left[1+\frac{\mu}{h^{2}}\left(t_{n}-\tau_{n-2}\right) \sin ^{2} \frac{\omega}{2}\right]^{2}} d \omega
\end{aligned}
$$




$$
\begin{aligned}
& <10 K \int_{0}^{\pi} \frac{\sin ^{4} \frac{\omega}{2}}{\left(1+K^{2} \sin ^{2} \frac{\omega}{2}\right)\left[1+\frac{\mu}{h^{2}}\left(t_{n}-\tau_{n-2}\right) \sin ^{2} \frac{\omega}{2}\right]^{2}} d \omega \\
& <\frac{10 K \pi\left[2 \sqrt{1+\frac{\mu}{h^{2}}\left(t_{n}-\tau_{n-2}\right)}+\sqrt{1+K^{2}}\right]}{2\left[1+\frac{\mu}{h^{2}}\left(t_{n}-\tau_{n-2}\right)\right]^{3 / 2} \sqrt{1+K^{2}}\left[\sqrt{1+\frac{\mu}{h^{2}}\left(t_{n}-\tau_{n-2}\right)}+\sqrt{1+K^{2}}\right]^{\circ}}
\end{aligned}
$$

So,

$$
\left|L\left(K ; t_{n}, \tau_{n-2}\right)\right|<\frac{C K h^{4}}{\sqrt{1+K^{2}}\left(t_{n}-\tau_{n-2}\right)^{3 / 2}\left(\sqrt{t_{n}-\tau_{n-2}}+K h\right)} .
$$

By applying the last inequality on the right hand side of (A.58), we obtain

$$
\text { (A. 61) }\left|J_{22}\left(K ; t_{n}, \tau_{n-2}\right)\right|<\frac{G}{h \sqrt{1+K^{2}} \sqrt{t_{n}-\tau_{n-2}}\left(\sqrt{t_{n}-\tau_{n-2}}+K h\right)} \text {. }
$$

Let us go to the general case $(p<n-1)$ 。

$$
\begin{aligned}
& \left|L\left(K ; t_{n}, \tau_{p-1}\right)\right| \\
& <\frac{3}{K} \int_{\frac{\pi}{K}}^{\pi} \prod_{q=p}^{n} \Lambda_{q}^{-1} \sin ^{2} \frac{\omega}{2} d \omega+\frac{8\left(t_{n}-\tau_{p-1}\right)}{K h^{2}} \int_{\frac{\pi}{K}}^{\pi} \prod_{q=p}^{n} \Lambda_{q}^{-1} \sin ^{4} \frac{\omega}{2} d \omega .
\end{aligned}
$$

We first apply (A.44). Then

(A. 62) $\left|L\left(K ; t_{n}, \tau_{p-1}\right)\right|<6 K M\left(K ; t_{n}, \tau_{p-1}\right)+\frac{16 K\left(t_{n}-\tau_{p-1}\right)}{h^{2}} N\left(K ; t_{n}, \tau_{p-1}\right)$ ， where

$$
M\left(K ; t_{n}, \tau_{p-1}\right)=\int_{0}^{\pi} \prod_{q=p}^{n} \Lambda_{q}^{-1} \frac{\sin ^{4} \frac{\omega}{2}}{1+K^{2} \sin ^{2} \frac{\omega}{2}} d \omega
$$

and

$$
N\left(K ; t_{n}, \tau_{p-1}\right)=\int_{0}^{\pi} \prod_{q=p}^{n} \Lambda_{q}^{-1} \frac{\sin ^{6} \frac{\omega}{2}}{1+K^{2} \sin ^{2} \frac{\omega}{2}} d \omega .
$$

Apply (A.31) and (A.34) for estimation of $M\left(K ; t_{n}, \tau_{p-1}\right)$. Then 


$$
\begin{aligned}
& M\left(K ; t_{n}, \tau_{p-1}\right) \\
& \quad<\int_{0}^{\pi} \frac{\sin ^{4} \frac{\omega}{2}}{\left(1+K^{2} \sin ^{2} \frac{\omega}{2}\right)\left[1+\frac{\mu}{h^{2}}\left(t_{n}-\tau_{p-1}\right) \sin ^{2} \frac{\omega}{2}\right]^{2}} d \omega \\
& =\frac{\left[2 \sqrt{1+\frac{\mu}{h^{2}}\left(t_{n}-\tau_{p-1}\right)}+\sqrt{1+K^{2}}\right]}{2\left[1+\frac{\mu}{h^{2}}\left(t_{n}-\tau_{p-1}\right)\right]^{3 / 2} \sqrt{1+K^{2}}\left[\sqrt{1+\frac{\mu}{h^{2}}\left(t_{n}-\tau_{p-1}\right)}+\sqrt{1+K^{2}}\right]^{2}} .
\end{aligned}
$$

Therefore

$$
\text { (A. 63) } 0<M\left(K ; t_{n}, \tau_{p-1}\right)<\frac{C h^{4}}{\left(t_{n}-\tau_{p-1}\right)^{3 / 2} \sqrt{1+K^{2}}\left(\sqrt{t_{n}-\tau_{p-1}}+K h\right)} .
$$

Apply next (A. 32) and (A.35) for estimation of $N\left(K ; t_{n}, \tau_{p-1}\right)$. Then

$$
\begin{aligned}
& N\left(K ; t_{n}, \tau_{p-1}\right) \\
& <\int_{0}^{\pi} \frac{\sin ^{6} \frac{\omega}{2}}{\left(1+K^{2}\right)\left[1+\frac{\mu}{h^{2}}\left(t_{n}-\tau_{p-1}\right) \sin ^{2} \frac{\omega}{2}\right]^{3}} d \omega \\
& =\frac{\pi}{\left[\sqrt{1+\frac{\mu}{h^{2}}\left(t_{n}-\tau_{p-1}\right)}+\sqrt{1+K^{2}}\right]^{3}}\left\{\frac{1}{\left[1+\frac{\mu}{h^{2}}\left(t_{n}-\tau_{p-1}\right)\right]^{3 / 2} \sqrt{1+K^{2}}}\right. \\
& \left.\quad+\frac{9}{8\left[1+\frac{\mu}{h^{2}}\left(t_{n}-\tau_{p-1}\right)\right]^{2}}+\frac{3 \sqrt{1+K^{2}}}{8\left[1+\frac{\mu}{h^{2}}\left(t_{n}-\tau_{p-1}\right)\right]^{5 / 2}}\right\} .
\end{aligned}
$$

Hence

$$
\text { (A. 64) } 0<N\left(K ; t_{n}, \tau_{p-1}\right)<\frac{C h^{6}}{\sqrt{1+K^{2}}\left(t_{n}-\tau_{p-1}\right)\left(\sqrt{t_{n}-\tau_{p-1}}+K h\right)} \text {. }
$$

Apply (A.63) and (A.64) on the right hand side of (A.62). We get

(A. 65) $\left|L\left(K ; t_{n}, \tau_{p-1}\right)\right|<\frac{C K h^{4}}{\sqrt{1+K^{2}\left(t_{n}-\tau_{p-1}\right)^{3 / 2}\left(\sqrt{t_{n}-\tau_{p-1}}+K h\right)}}$.

Further, apply (A.65) on the right hand side of (A. 58). Then

(A. 66) $\left|J_{22}\left(K ; t_{n}, \tau_{p-1}\right)\right|<\frac{C}{h \sqrt{1+K^{2}} \sqrt{t_{n}-\tau_{p-1}}\left(\sqrt{t_{n}-\tau_{p-1}}+K h\right)}$.

Combining (A.57), (A.61) and (A.66), we obtain, for $p<n$, 


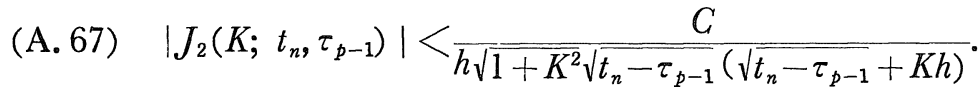

Finally, apply (A.53) and (A.67) upon (A.49). Then, we also have (A. 68) $\left|J\left(K ; t_{n}, \tau_{p-1}\right)\right|<\frac{G}{h \sqrt{1+K^{2}} \sqrt{t_{n}-\tau_{p-1}}\left(\sqrt{t_{n}-\tau_{p-1}}+K h\right)}$

for $p<n$ and $K \neq 0$. Thus, we have proved Lemma A. 5 completely.

\section{A. 8. Estimation of $\boldsymbol{u}_{\boldsymbol{x}}$}

We now use Lemma A. 4 and A. 5 to estimate the right hand sides of (A.25)-(A.27). We then get the following estimations.

$$
\begin{aligned}
& \left|G\left(x_{r}, \xi_{j} ; t_{n}, 0\right)_{x}\right| \\
& <\left|I\left(r+j ; t_{n}, 0\right)\right|+\left|I\left(r-j ; t_{n}, 0\right)\right|+h\left[\left|J\left(r+j ; t_{n}, 0\right)\right|+\left|J\left(r-j ; t_{n}, 0\right)\right|\right] \\
& <C\left\{\frac{1}{\sqrt{t_{n}}\left(\sqrt{t_{n}}+x_{r}+\xi_{j}\right)}+\frac{1}{\sqrt{t_{n}}\left(\sqrt{t_{n}}+\left|x_{r}-\xi_{j}\right|\right)}\right. \\
& \left.\quad+\frac{h}{\left(x_{r}+\xi_{j}\right) \sqrt{t_{n}}\left(\sqrt{t_{n}}+x_{r}+\xi_{j}\right)}+\frac{h}{\sqrt{h^{2}+\left(x_{r}-\xi_{j}\right)^{2}} \sqrt{t_{n}}\left(\sqrt{t_{n}}+\left|x_{r}-\xi_{j}\right|\right)}\right\} .
\end{aligned}
$$

Hence

(A. 69) $\left|G\left(x_{r}, \xi_{j} ; t_{n}, 0\right)_{x}\right|<C\left\{\frac{1}{\sqrt{t_{n}}\left(\sqrt{t_{n}}+x_{r}+\xi_{j}\right)}+\frac{1}{\sqrt{t_{n}}\left(\sqrt{t_{n}}+\left|x_{r}-\xi_{j}\right|\right.}\right\}$.

Next

(A. 70) $\left|G\left(x_{r}, 0 ; t_{n}, \tau_{p-1}\right)_{x \xi}\right|<2\left[\left|J\left(r+1 ; t_{n}, \tau_{p-1}\right)\right|+\left|J\left(r ; t_{n}, \tau_{p-1}\right)\right|\right]$

$$
<\frac{C}{x_{r} \sqrt{t_{n}-\tau_{p-1}}\left(\sqrt{t_{n}-\tau_{p-1}}+x_{r}\right)} \quad\left(\text { for } x_{r}>0\right)
$$

and

$$
\text { (A. 71) } \begin{aligned}
&\left|G\left(x_{r}, X ; t_{n}, \tau_{p-1}\right)_{\xi_{x}}\right|<2 {\left[\left|J\left(J+r ; t_{n}, \tau_{p-1}\right)\right|\right.} \\
&\left.+\left|J\left(J-r-1 ; t_{n}, \tau_{p-1}\right)\right|\right] \\
&\left.<\frac{G}{\left(X-x_{r}\right) \sqrt{t_{n}-\tau_{p-1}}\left(\sqrt{t_{n}-\tau_{p-1}}+X-x_{r}\right)} \quad \text { (for } x_{r}<X\right) .
\end{aligned}
$$

We are ready to estimate $u_{x}$. Assume that data are bounded: (A. 72) $\left|\phi_{j}\right|,\left|f^{p}\right|$ and $\left|g^{p}\right|<B \quad\left(0 \leqq x_{j} \leqq X, 0<t_{p}<T\right)$, where $B$ is a positive constant. From (A. 17), we have 


$$
\begin{aligned}
\left|\left(u_{r}^{n}\right)_{x}\right|< & B\left[\sum_{j=1}^{J-1} h\left|G\left(x_{r}, \xi_{j} ; t_{n}, 0\right)_{x}\right|+\sum_{p=1}^{n} k_{p}\left|G\left(x_{r}, 0 ; t_{n}, \tau_{p-1}\right)_{\xi x}\right|\right. \\
& \left.+\sum_{p=1}^{n} k_{p}\left|G\left(x_{r}, \xi_{j} ; t_{n}, \tau_{p-1}\right)_{\xi_{x}}\right|\right] .
\end{aligned}
$$

Apply (A. 69)-(A.71) on the last right hand side. Then

$$
\begin{aligned}
& \left|\left(u_{r}^{n}\right)_{x}\right|<C\left[\frac{1}{\sqrt{t_{n}}} \int_{0}^{X} \frac{d \xi}{\sqrt{t_{n}}+x_{r}+\xi}+\frac{1}{\sqrt{t_{n}}} \int_{0}^{X} \frac{d \xi}{\sqrt{t_{n}}+\left|x_{r}-\xi\right|}\right. \\
& \left.\quad+\frac{1}{x_{r}} \int_{0}^{t_{n}} \frac{d \tau}{\sqrt{t_{n}-\tau}\left(\sqrt{t_{n}-\tau}+x_{r}\right)}+\frac{1}{X-x_{r}} \int_{0}^{t_{n}} \frac{d \tau}{\sqrt{t_{n}-\tau}\left(\sqrt{t_{n}-\tau}+X-x_{r}\right)}\right] \\
& \quad<C\left[\frac{1}{\sqrt{t_{n}}} \log \left(1+\frac{X}{\sqrt{t_{n}}}\right)+\frac{1}{x_{r}} \log \left(1+\frac{\sqrt{t_{n}}}{x_{r}}\right)+\frac{1}{X-x_{r}} \log \left(1+\frac{\sqrt{t_{n}}}{X-x_{r}}\right)\right] .
\end{aligned}
$$

Thus, we have arrived at the following theorem:

Theorem A.6. Let $u$ be a solution of the problem (A.1) with the assumption (A. 72) in such a case that

$$
\min _{1 \leqq n \leqq N} k_{n}>\mu \max _{1 \leqq n \leqq N} k_{n} \quad \text { for } t_{N}<T
$$

always holds, where $\mu$ is a constant, $0<\mu<1$. Then

$$
\begin{aligned}
\left|\left(u_{j}^{n}\right)_{x}\right|<C\left[\frac{1}{\sqrt{t_{n}}} \log (1\right. & \left.+\frac{X}{\sqrt{t_{n}}}\right)+\frac{1}{x_{j}} \log \left(1+\frac{\sqrt{t_{n}}}{x_{j}}\right) \\
& \left.+\frac{1}{X-x_{j}} \log \left(1+\frac{\sqrt{t_{n}}}{X-x_{j}}\right)\right]
\end{aligned}
$$

holds uniformly in $h$ for $t_{n}<T$.

As easily seen, the last estimation for $u_{x}$ is weaker than the estimation of Bernstein type usally accepted, just by some logarithmic factors. It may be due to the method used by us.

\section{A. 9. Uniform Boundedness of Difference Quotients}

We define a 'parabolic' region $\Omega$ by

$$
\Omega=\{0<x<X, 0<t \leqq T\}
$$

and its boundary $\partial \Omega$ by

$$
\partial \Omega=\{x=0,0 \leqq t \leqq T\} \cup\{x=X, 0 \leqq t \leqq T\} \cup\{0 \leqq x \leqq X, t=0\} .
$$

Take that $\Omega(t)$ denotes a section of $\Omega$ at a time $t$. We further define a sequence of sectional regions and their boundaries as follows: $\Omega_{n}^{n}$ 
is a set of all the mesh points with space mesh width $h$ which are contained in $\Omega\left(t_{n}\right)$, together with respective two neighbouring mesh points. The remained mesh points in $\Omega\left(t_{n}\right)$ constitute its boundary $\omega_{h^{\circ}}^{n}$

Now, we can rewrite problem (A. 1) as follows:

$$
\begin{array}{lll}
\left(u_{j}^{n}\right)_{\bar{t}}=\left(u_{j}^{n}\right)_{x \bar{x}} & \text { for } x_{j} \in \Omega_{h}^{n} \quad(n=1,2, \ldots), \\
u_{j}^{n}=f_{j}^{n} & \text { for } x_{j} \in \omega_{h}^{n} \quad(n=1,2, \ldots), \\
u_{j}^{0}=\phi_{j} & \text { for } x_{j} \in \Omega_{h^{\circ}}^{0} &
\end{array}
$$

Theorem A. 7. Suppose that $\left\{f_{j}^{n}\right\}$ and $\left\{\phi_{j}\right\}$ are bounded uniformly in $h$ and $\left\{k_{n}\right\}$, for $t_{n}<T$. Then, a sequence of solutions of (A. 75) with $h \rightarrow 0$ is uniformly bounded in $\Omega$, and all kinds of sequences of difference quotients of $u_{j}^{n}$ are uniformly bounded on any compact set $\Omega^{*}$ contained in $\Omega$, respectively.

Proof. The uniform boundedness of $\left\{u_{j}^{n}\right\}$ itself follows immediately from the maximum principle. In order to prove the latter part of the theorem, we take a sequence of polygonal regions $\Omega_{k}^{*}(k=1,2,3$, ...) such that

$$
\Omega^{*} \subset \cdots \cdots \subset \Omega_{k+1}^{*} \subset \Omega_{k}^{*} \cdots \cdots \subset \Omega_{1}^{*} \subset \Omega,
$$

where every $\Omega_{k+1}^{*}$ is strictly inside of $\Omega_{k}^{*}$ and they are all composed of a number of rectangular subregions with sides parallel to the corresponding coordinate axes. Clearly, $\left\{u_{j}^{n}\right\}$ are uniformly bounded on boundaries of all rectangulars of $\Omega_{1}^{*}$. Apply Theorem A. 6 on all restricted problems in respective rectangulars. We then find that $\left\{\left(u_{j}^{n}\right)_{x}\right\}$ are uniformly bounded all over $\Omega_{2}^{*}$. Similarly, we again find from the last fact proven that $\left\{\left(u_{j}^{n}\right)_{x i x}\right\}$ also are uniformly bounded on $\Omega_{3}^{*}$, and hence so are $\left\{\left(u_{j}^{n}\right)_{i}\right\}$. By repeating this discussion for $\left\{\Omega_{k}^{*}, k=4,5, \ldots\right\}$, we are led to the concerned statement of the theorem.

\section{Appendix $\mathbb{B}$}

Here, we will give some facts about several kinds of function class for completeness of the present paper. They are already known and are given, for example, in Nikolskii's book [7] in the more general 
frame. We will state them without proof, and moreover only in such a restricted frame that was necessary for our problem in $\S 3$.

In $R^{2}$, we consider the region

$$
\Omega=\left\{0<x_{1}<y\left(x_{2}\right), 0<x_{2}<T\right\},
$$

where $y\left(x_{2}\right)$ is given and continuously differentiable, and its derivative has a non-zero limit $y^{\prime}(0)$. Let us introduce Sobolev class $\mathrm{W}_{2}^{r}(\Omega)$ $\left(r=\left(r_{1}, r_{2}\right)\right)$ with the norm defined by

$$
\|u\|_{W_{2}^{r}(\Omega)}^{2}=\|u\|_{L_{2}(\Omega)}^{2}+\left\|\frac{\partial^{r_{1}} u}{\partial x_{1}^{r_{1}}}\right\|_{L_{2}(\Omega)}^{2}+\left\|\frac{\partial^{r_{2}} u}{\partial x_{2}{ }^{r_{2}}}\right\|_{L_{2}(\Omega)}^{2} .
$$

I) Any element $u \in W^{2,1}(\Omega)$ has an extension $u \in W^{2,1}\left(R^{2}\right)$ all over the space $R^{2}$.

Let us introduce new variables by

$$
\xi_{1}=y\left(x_{2}\right)-x_{1}, \quad \xi_{2}=x_{2},
$$

where it is assumed that $y\left(x_{2}\right)$ is already extended beyond the original interval $(0, T)$ so that $y^{\prime}\left(x_{2}\right)$ is bounded and continuously differentiable in $-\infty<x_{2}<\infty$. Put

$$
v\left(\xi_{1}, \xi_{2}\right)=u\left(\xi_{1}+y\left(\xi_{2}\right), \xi_{2}\right) .
$$

II) $v \in W_{2}^{2,1}\left(R^{2}\left(\xi_{1}, \xi_{2}\right)\right)$.

Let us introduce one more class: Hardy class $H_{\xi_{2}}^{0}\left(R_{\xi_{2}}^{1}\right)$. It is a Banach space of functions of one variable $\xi_{2}$ with the following norm:

$$
\|w\|_{H_{\xi_{2}}^{\rho}\left(R_{\xi_{2}}^{1}\right)}=\|w\|_{L^{2}\left(R_{\xi_{2}}^{1}\right)}+\sup \left[h^{-(\rho-\bar{\rho})}\left\|\Delta_{\xi_{2} h} \frac{\partial^{\bar{\rho}_{w}}}{\partial \xi_{2}^{\bar{\rho}}}\right\| L_{L^{2}\left(R_{\xi_{2}}^{1}\right)}\right],
$$

where $\rho=\bar{\rho}+\alpha, \bar{\rho}$ is an integer and $0<\alpha<1$, ahd $\Delta_{\bar{\xi}_{2} h}$ is a forward difference operator of the first order:

$$
\Lambda_{\xi_{2} h} w\left(\xi_{2}\right)=w\left(\xi_{2}+h\right)-w\left(\xi_{2}\right) \text {. }
$$

Imbedding Theorem. For any pair of non-negative integers $r=\left(r_{1}, r_{2}\right)$,

$$
W_{2}^{r}\left(R^{2}\right) \rightarrow H_{\xi_{2}}^{\rho}\left(R_{\xi_{2}}^{1}\right), \quad \rho=\left(1-\frac{1}{2 r_{2}}\right) r_{1} .
$$

It means that $v\left(\xi_{1}, \xi_{2}\right) \in W_{2}^{r}\left(R^{2}\right)$ has a unique trace

$$
w\left(\xi_{2}\right)=v\left(+0, \xi_{2}\right)=\lim _{\xi_{2} \rightarrow+0} v\left(\xi_{1}, \xi_{2}\right)
$$

defined for almost every $\xi_{2}$, on $R_{\xi_{2}}^{1}$, lying in $H_{\xi_{2}}^{o}\left(R_{\xi_{2}}^{1}\right)$, and that the inequality 


$$
\|; w\|_{H_{\xi_{2}}^{\rho}\left(R_{\xi_{2}}^{1}\right)}<C\|v\|_{W_{2}^{r}\left(R^{2}\right)}
$$

is satisfied, where $C$ does not depend on $v$.

Especially, we hence have

III)

$$
w_{2}^{2,1}\left(R^{2}\right) \rightarrow H_{\xi_{2}}^{3 / 4}\left(R_{\xi_{2}}^{1}\right) .
$$

From I-III, we can conclude from $u \in W_{2}^{2,1}(\Omega)$ that

$$
\sup \left[h^{-3 / 4}|| \Delta_{\xi_{2} h^{2}} w \|_{L^{2}\left(R_{\xi_{2}}^{1}\right)}\right]<K
$$

with a constant $K$, and hence

$$
\left\|u\left(y\left(x_{2}+h\right), x_{2}+h\right)-u\left(y\left(x_{2}\right), x_{2}\right)\right\|_{L^{2}\left(R_{x_{2}}^{1}\right)}<K h^{3 / 4}
$$

for all $h>0$. The last inequality itself was used in $\S 3$.

\section{Acknowledgement}

One phase problem having the same physical background was already dealt with by Mr. R. Kobayashi [8]. He also helped the author to refine the proof of Lemma A. 1. The author thanks to him.

\section{References}

[1] Nogi, T. and Yoshida, N., Approximate Solutions of Mathematical Models of Supercooling Solidification, Memoirs of the Faculty of Engineering, Kyoto University, 43, Part 4, (1981), 388-396.

[2] Fulks, W. and Guenther, R. B., A Free Boundary Problem and an Extension of Muskat's Model, Acta Mathematical, 122, (1969), 273-300.

[3] Pawlow, I, On Some Properties of Two-Layer Parabolic Free Boundary Value problems, Control and Cybernetics, 7, No. 4, (1978), 19-37.

[4] Nogi, T., A Difference Scheme for Solving Two Phase Stefan Problem of Heat Equation, Publ. RIMS, Kyoto Univ., 16 (1980), 388-396.

[5] Petrowsky, I. G., Partial Differential Equations, London Iliffe Books Ltd., 1967.

[6] Friedman, A., Remarks on the maximum principle for parabolic equations and its applications, Pacific J. Math., 8, No. 2, (1958).

[7] Nikol'skii, S. M., Approximation of Functions of Several Variables and Imbedding Theorems, Springer-Verlag Berlin Heiderberg New York 1975.

[8] Kobayashi, R., A Mathematical One-Dimensional One-Phase Model of Supercooling Solidification, Publ. RIMS, Kyoto Univ., 19 (1983), 327-344. 
